Portland State University

PDXScholar

Winter 3-22-2019

\title{
Localized Photoemission in Triangular Gold Antennas
}

Christopher M. Scheffler

Portland State University

Follow this and additional works at: https://pdxscholar.library.pdx.edu/open_access_etds

Part of the Physics Commons

Let us know how access to this document benefits you.

Recommended Citation

Scheffler, Christopher M., "Localized Photoemission in Triangular Gold Antennas" (2019). Dissertations and Theses. Paper 4866.

https://doi.org/10.15760/etd.6742

This Thesis is brought to you for free and open access. It has been accepted for inclusion in Dissertations and Theses by an authorized administrator of PDXScholar. Please contact us if we can make this document more accessible: pdxscholar@pdx.edu. 


\section{Localized Photoemission in Triangular Gold Antennas}

by

Christopher M. Scheffler

A thesis submitted in partial fulfillment of the requirements for the degree of

Master of Science

in

Physics

Thesis Committee:

Rolf Könenkamp, Chair

Pui-Tak Leung

Erik Sánchez

Portland State University

2019 
(C) 2019 Christopher M. Scheffler 


\begin{abstract}
With the development of ultra-fast laser technology, several new imaging techniques have pushed optical resolution past the diffraction limit for traditional lightbased optics. Advancements in lithography have enabled the straightforward creation of micron- and nanometer-sized optical devices. Exposing metal-dielectric structures to light can result in surface plasmon excitation and propagation along the transition interface, creating a surface plasmon polariton (SPP) response. Varying the materials or geometry of the structures, the plasmonic response can be tailored for a wide range of applications.

Photoemission electron microscopy (PEEM) has been used to image excitations in micron-sized plasmonic devices. With PEEM, optical responses can be characterized in detail, aiding in the development of new types of plasmonic structures and their applications. We show here that in thin, triangular gold platelets SPPs can be excited and concentrated within specific regions of the material (thickness $\sim 50 \mathrm{~nm}$ ); resulting in localized photoemission in areas of high electric field intensity. In this regard, the platelets behave as receiver antennas by converting the incident light into localized excitations in specific regions of the gold platelets. The excited areas can be significantly smaller than the wavelength of the incident light $(\lambda \leq 1 \mu \mathrm{m})$. By varying the wavelength of the light, the brightness of the excited spots can be changed and by varying the polarization of the light, the brightness and position can be changed, effectively switching the photoemission on or off for a specific region within the triangular gold structure.
\end{abstract}


In this work, the spatial distribution of surface plasmons and the imaging results from photoemission electron microscopy are reproduced in simulation using finite element analysis (FEA). In addition, we show that electromagnetic theory and simulation enable a detailed and quantitative analysis of the excited SPP modes, an explanation of the overall optical responses seen in PEEM images, and prediction of new results. 


\section{Dedication}

This thesis is dedicated to my mother and father: Catrina and Kevin Scheffler, for cultivating my curiosity of the physical world and demonstrating the value of education; to my siblings: Cole and Rachael Scheffler, to encourage them to always continue learning and improving themselves; and to my partner: Rebecca Jane Langer, who challenged me to strive for greatness and made me the person I am today. 


\section{Acknowledgments}

I would like to thank Dr. Rolf Könenkamp for serving as my research advisor at Portland State University and the chair of my Master's Thesis Committee. In addition, Dr. Pui-Tak Leong and Dr. Erik Sànchez for serving as Committee members and advisors in the creation of this thesis.

Academically, I would like to thank Theodore Stenmark, Xiang Wei, and Dr. Robert Word for instructing me in and providing reference materials for the areas of photonics, plasmonics, photoemission electron microscopy, and software modeling. They have been incredible resources for my graduate research and coursework. In addition, the PEEM micrographs used for this research were acquired by Dr. Word prior to my arrival.

Personally, I would like to thank Jon, Kyle, and Krista for their support and patience during this process and for motivating me to understand the material well enough to explain it coherently to non-physicists.

Professionally, I would like to thank Rick, Shida, Roy, John, and Mike for their encouragement, mentorship, and support in helping me become a successful research scientist, but even more so in leaving it all to return to academia for graduate studies.

Lastly, this research was supported in part by the US Department of Energy's Basic Science Office under Contract No. DE-FG02-13ER46406 and the US Department of Defense's Science, Mathematics, and Research for Transformation (SMART) Scholarship program. 


\section{Table of Contents}

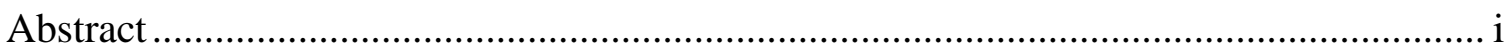

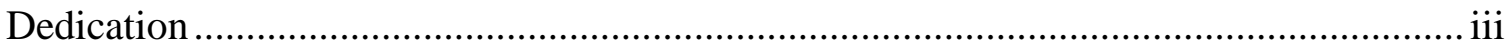

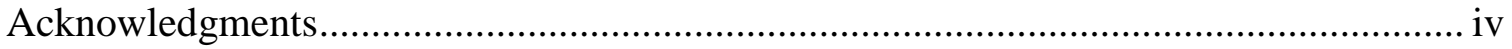

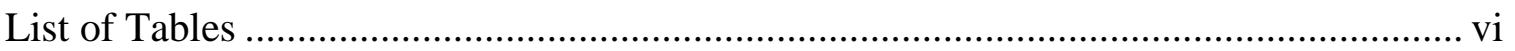

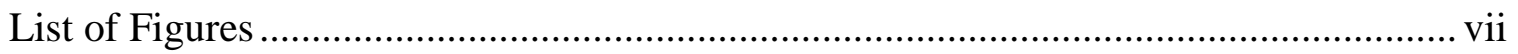

Glossary of Terms, Abbreviations, and Symbols ........................................................ xii

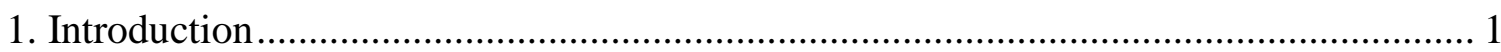

2. Photoemission Electron Microscopy (PEEM) for Plasmonics ..................................... 4

2.1 Background Theory and PEEM Overview ........................................................... 4

2.2 Experiment and Sample Details ..................................................................... 9

3. Finite Element Method (FEM) Based Modeling ............................................... 12

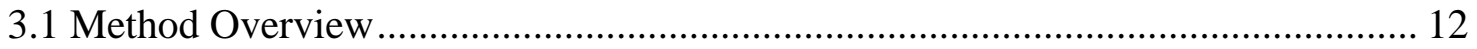

3.2 Parameter Details .......................................................................................... 14

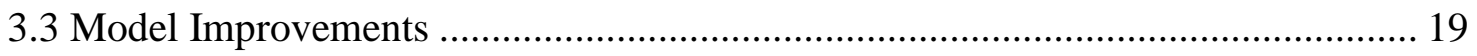

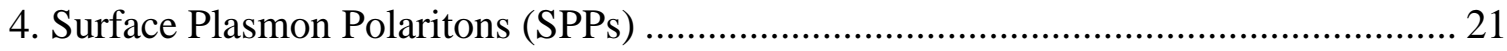

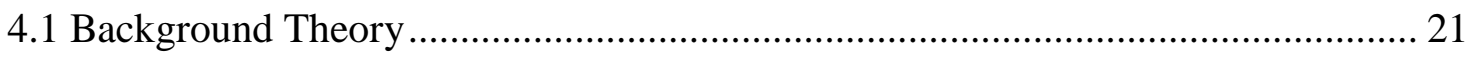

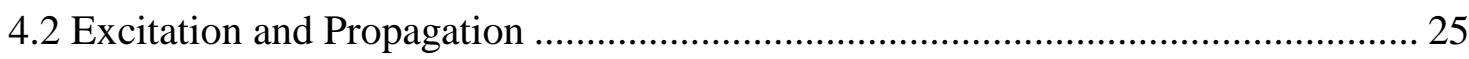

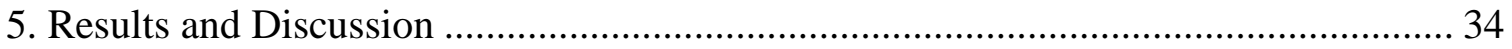

5.1 Experimental Results.............................................................................. 34

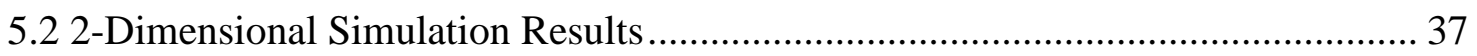

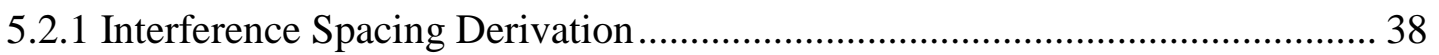

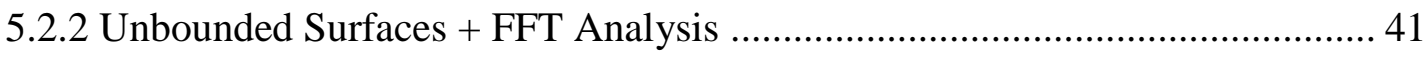

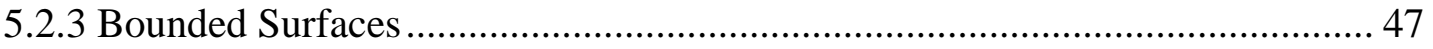

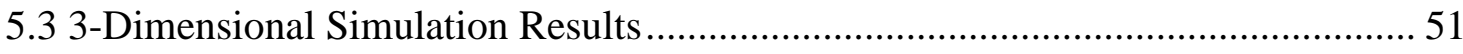

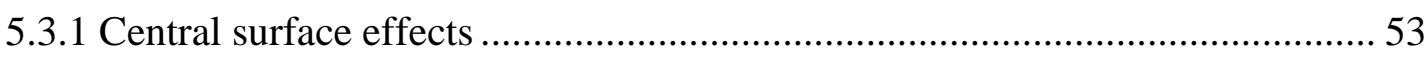

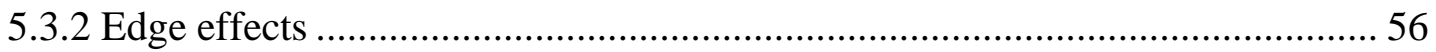

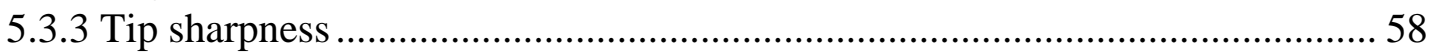

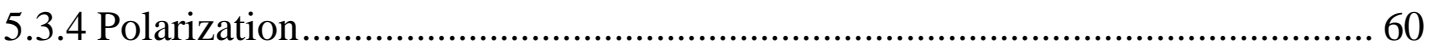

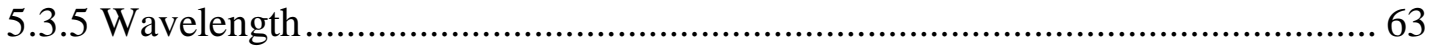

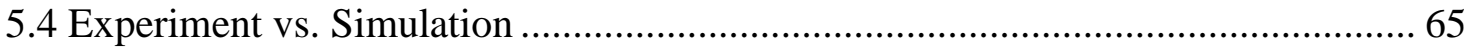

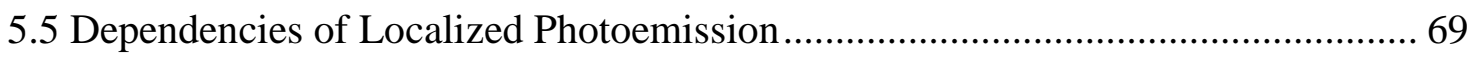

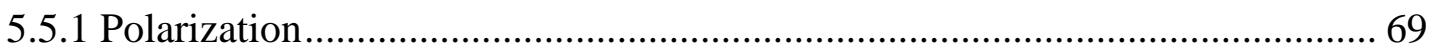

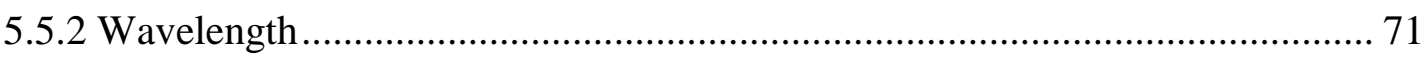

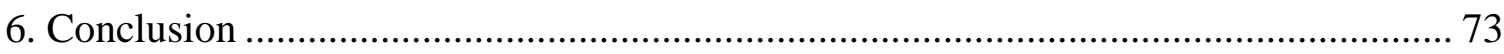

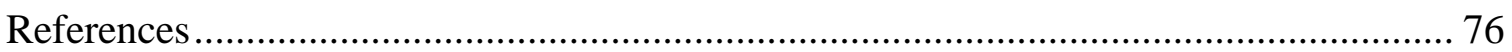




\section{List of Tables}

Table 1: Major parameters evaluated for the 3D COMSOL gold-ITO-glass model....... 17

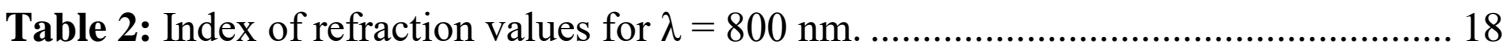

Table 3: Calculated speed (s), wavelength $(\lambda)$, and propagation length (L) for SPP modes of non-hybridized single-interfaces of vacuum-gold and gold-ITO and hybridized multilayer vacuum-gold-ITO interfaces with $\lambda=800 \mathrm{~nm}$ incident light. The interference spacing values are for $\lambda=800 \mathrm{~nm}$ light obliquely incidence at $\theta=60^{\circ}$....................... 33

Table 4: Theoretical and experimental values for interference periodicities at the gold and ITO surfaces for thick $(1 \mu \mathrm{m})$ and thin $(50 \mathrm{~nm})$ gold layers. 


\section{List of Figures}

Figure 1: Illustration of example PEEM optics (simplified), detailing the laser illumination, sample, lenses, deflectors, phosphor screen, and CCD camera.

Figure 2: Photographs of the PEEM components (a) and the laser system (b, c). Labeled components: (1) sample loadlock, (2) sample preparation chamber, (3) objective lens, (4) transfer lenses, (5) diode electron mirror, (6) projection lenses; (7) phosphor screen,

(8) CCD camera, (9) Ar-ion CW laser, (10) Mai Tai Ti-Sapphire pulsed laser,

(11) second / third harmonics generator, (12) fourth harmonic generator,

(13) tunable waveplate, and (14) spatial modulator.

Figure 3: Illustrations of the: (a) PEEM specimen stub, depicting oblique (from above) and normal (below) incidence [26]; (b) incidence angle $\theta$; and (c) polarization angle $\varphi .10$

Figure 4: Illustration of an example gold (Au)-ITO-glass sample from (a) cross-sectional and (b) top-down views.

Figure 5: 3D COMSOL model of a gold-ITO-glass sample with a surrounding vacuum region: (a) gold region selected in green and (b) simulation results of the time-averaged electric field at the gold surface for $\lambda=800 \mathrm{~nm}$ obliquely $\left(\theta=60^{\circ}\right)$ incident light. ....... 13

Figure 6: (a) Cross-sectional view of a 2D COMSOL model of a gold-ITO-glass sample with the layers labeled and the gold region highlighted in green; (b) simulation results of the normalized electric field for $\lambda=800 \mathrm{~nm}$ light incident obliquely from above at ...... 14

Figure 7: 3D COMSOL model of a gold-ITO-glass sample with a surrounding vacuum region with the tetrahedral meshing displayed: (a) entire model and (b) magnified view of the tip region of the triangular gold layer (highlighted in purple). 16

Figure 8: Graph of the reflection of a plane wave vs. incidence angle for a $1^{\text {st }}$ order SBC.

Figure 9: Illustration of the common planar waveguide geometry, with propagation in the $\mathrm{x}$-direction.

Figure 10: Illustration of the geometry and real portion of the electric field distribution for a single metal-dielectric interface. 
Figure 11: Illustration of the geometry and real portion of the electric field distribution for a multi-interface structure with a thick and thin metal layer. Note the two SPP modes are independent for the thick metal region but become hybridized for a thin metal region.

Figure 12: Graphical solutions for the symmetric $\left(\mathrm{s}_{\mathrm{b}}\right)$ and anti-symmetric $\left(\mathrm{a}_{\mathrm{b}}\right)$ SPP modes for $\lambda=800 \mathrm{~nm}$ light incident onto a vacuum-gold-ITO multi-layer interface for gold of thickness $T=\{60,70,80,90,100 \mathrm{~nm}\}$. The solid lines represent solutions to the real portion of the dispersion relationship and the dotted lines the imaginary portions.

The plots were calculated via Mathematica.

Figure 13: Graphical solutions for the anti-symmetric SPP modes for $\lambda=800 \mathrm{~nm}$ light incident onto a vacuum-gold-ITO multi-layer interface for gold of thickness $\mathrm{T}=\{60,70,80,90,100 \mathrm{~nm}\}$. The solid lines represent solutions to the real portions of the dispersion relationship and the dotted lines the imaginary portions.

Figure 14: Graph of the propagation length vs. gold thickness for the anti-symmetric (green, solid) and the symmetric (blue, dotted) SPP modes excited by $\lambda=800 \mathrm{~nm}$ light on a planar vacuum-gold-ITO sample. Above $\sim 100 \mathrm{~nm}$ the two hybridized SPP modes remain constant with increasing gold thickness

Figure 15: PEEM micrograph of an array of gold microstructures excited with $\lambda=244 \mathrm{~nm} \mathrm{CW}$ light incident at $\theta=60^{\circ}$. Micrograph provided by Dr. Robert C. Word. 34

Figure 16: PEEM micrographs of gold microstructures excited by $\lambda=800 \mathrm{~nm}$ light incident at $\theta=60^{\circ}$ for TM- (a) original, (b) digitally magnified and colored - and TE polarizations $\{(\mathrm{c}),(\mathrm{d})\}$. The magnified triangular platelet is equilateral with a length of $\sim 2.9 \mu \mathrm{m}$. Contrast ranges for the micrographs: $(\mathrm{a} / \mathrm{b}) \equiv \mathrm{C}_{\mathrm{ab}}=\{1256: 40875\}$, (c) $=0.9 \cdot \mathrm{C}_{\mathrm{ab}}$, and $(\mathrm{d})=0.5 \cdot \mathrm{C}_{\mathrm{ab}}$. Note: TE excitation shows no photoemission here. .. 35

Figure 17: Normal incidence $\left(\theta=0^{\circ}\right)$ PEEM micrographs of triangular gold platelets for $\lambda=800 \mathrm{~nm}$ light with (a) $\varphi=0^{\circ}$ and (b) $\varphi=90^{\circ}$ polarizations. Note: The micrographs are presented as the $3^{\text {rd }}$ root of the photoemission yield. 36

Figure 18: (a) Cross-sectional view of a 2D COMSOL model of a vacuum-gold-ITOglass sample with the layers labeled and the gold region highlighted in green and (b) a top down illustration of the triangular gold structure with the 2D plane of simulation marked with a dashed line (red). 
Figure 19: Illustration of the experiment geometry of a vacuum-gold SPP (blue) traveling along the gold surface to interfere with light of wavelength $\lambda$ (red) incident at an angle relative to the surface normal of $\theta . \mathrm{x}_{1}$ is the distance traveled by the SPP between the edge of the gold region and the location of the next interference maximum with the incident light and $\mathrm{x}_{2}$ is the angled distance traveled by the incident light between the edge of the gold region and the sample surface. 38

Figure 20: 2D simulation results of the time-averaged electric field for $\lambda=800 \mathrm{~nm}$ light obliquely incident at $\theta=60^{\circ}$ to the surface normal with TM polarization for a thick gold layer $(\mathrm{T}=1 \mu \mathrm{m})$ on top of a planar ITO-glass stack (surrounded by a vacuum region): (a) simulation results for the full model and (b) a zoomed image of the surface of the gold layer. The $\sim 5.2 \mu \mathrm{m}$ interference spacing between a SPP mode excited at the edge of the gold layer and the incident light calculated above is visible along the gold surface....... 41

Figure 21: (a) Graph of the time-averaged electric field squared at the gold surface vs. distance from the boundary of the model for a $50 \mu \mathrm{m}$ long gold platelet exposed to $\lambda=800 \mathrm{~nm}$ light with oblique incidence $\left(\theta=60^{\circ}\right)$ and TM polarization for thin (50 nm; green, solid) and thick ( $1 \mu \mathrm{m}$; blue, dotted) gold layers; (b) FFT analysis of the electric field data plotted in (a). A single periodicity is visible for both $50 \mathrm{~nm}$ and $1 \mu \mathrm{m}$ thick gold layers at $\sim 5.2 \mu \mathrm{m}$, which is attributed to the symmetric SPP mode interfering with the incident light. FFT data calculated via Mathematica. The normal distribution (black, dashed) was fit to the data sets before a $y_{\text {shift }}=0.15$ was applied to the $1 \mu \mathrm{m}$ curve.

Figure 22: FFT analysis of the electric field data from Figure 21.a taken at the ITO surface. A single periodicity is visible for the $1 \mu \mathrm{m}$ thick gold layer (blue, dotted) at $\sim 1.7 \mu \mathrm{m}$ and two for the $50 \mathrm{~nm}$ thick gold (green, solid) at $\sim 5.5 \mu \mathrm{m}$ and $860 \mathrm{~nm}$. FFT data calculated via Mathematica.

Figure 23: Graph of the time-averaged electric field at the gold surface vs. distance from the front edge of the gold layer for thin $(50 \mathrm{~nm}$; green, solid) and thick (1 $\mu \mathrm{m}$; blue, dotted) gold platelets of varying length $(\mathrm{L}=2.1 \mu \mathrm{m}-8.0 \mu \mathrm{m})$ exposed to 47

Figure 24: Simulation results of the time-averaged electric field squared at the gold surface for a $3 \mu \mathrm{m}$ long gold platelet exposed to $\lambda=800 \mathrm{~nm}$ light with $\varphi=90^{\circ}$ polarization for: (a) oblique incidence $\left(\theta=60^{\circ}\right)$ and (b) normal incidence $\left(\theta=0^{\circ}\right)$. (c) Graph of the electric field squared vs. distance from the boundary of the model for oblique incidence. Contrast ranges of the micrographs are: 
Figure 25: Graph of the time-averaged electric field squared vs. distance from the boundary of the model for a $3 \mu \mathrm{m}$ long gold platelet exposed to $\lambda=800 \mathrm{~nm}$ light with oblique incidence $\left(\theta=60^{\circ}\right)$ and TM polarization for a series of vertical distances into the gold region (i.e. $0[\mathrm{~nm}]=$ gold surface, $25[\mathrm{~nm}]=$ midpoint for $50[\mathrm{~nm}]$ thick layer).... 54

Figure 26: (a) Illustrations of the three combinations of rods and a triangular platelet; (b) a graph of the simulated time-averaged electric field squared at the gold surface vs. distance from the boundary of the model for $\lambda=800 \mathrm{~nm}$ light with oblique incidence $\left(\theta=60^{\circ}\right)$ and TM polarization for the four structures; and (c) the same data graphed for only the triangular platelet (Y-offset: 0.6; green, solid) and the "Triple + back" rod arrangement (black, dotted), showing identical electric field responses. The gold platelet and rod structures are $3 \mu \mathrm{m}$ in length.

Figure 27: (a) Illustrations of the three combinations of rods and a triangular platelet; (b) a graph of the simulated time-averaged electric field squared at the gold surface vs. distance from the boundary of the model for $\lambda=800 \mathrm{~nm}$ light with oblique incidence $\left(\theta=60^{\circ}\right)$ and TM polarization for the four structures; and (c) the same data graphed for only the triangular platelet (Y-offset: 0.6; green, solid) and the "Dual + back" rod arrangement (black, dotted), showing identical electric field responses. The gold platelet and rod structures are $3 \mu \mathrm{m}$ in length.

Figure 28: Illustration of a gold rod (highlighted in green) with the tip angle labeled. .. 58

Figure 29: Graph of the simulated time-averaged electric field squared at the gold surface vs. distance from the boundary of the model for $\lambda=800 \mathrm{~nm}$ light with: oblique incidence $\left(\theta=60^{\circ}\right)$ and TM polarization for single angled rods with varying internal tip angle between $\left\{30^{\circ}: 90^{\circ}\right\}$. Magnified view of the tip region. Note: the data is normalized such that the peak electric field intensity at the tip for the $30^{\circ}$ internal angle is 1 . The electric field outside of the region displayed is approximately zero for all internal angles.

Figure 30: Simulation results of the time-averaged electric field squared at the gold surface for $3 \mu \mathrm{m}$ long gold platelets exposed to $\lambda=800 \mathrm{~nm}$ light with: oblique incidence $\left(\theta=60^{\circ}\right)$ (a) TM and (b) TE and normal incidence $\left(\theta=0^{\circ}\right)$ (c) $\varphi=90^{\circ}$ and (d) $\varphi=0^{\circ}$ polarizations. Contrast ranges of the micrographs are:

Figure 31: Graph of the simulated time-averaged electric field squared at the gold surface vs. distance from the boundary of the model for a $3 \mu \mathrm{m}$ long gold platelet exposed to $\lambda=800 \mathrm{~nm}$ light with: oblique incidence $\left(\theta=60^{\circ}\right)$ for $\mathrm{TM}$ 
Figure 32: Graph of the simulated time-averaged electric field squared at the gold surface of the rear tip vs. polarization angle for obliquely incident light $\left(\theta=60^{\circ}\right)$ for $\varphi=\left\{0: 360^{\circ}\right\}$

Figure 33: Simulation results of the time-averaged electric field squared at the gold surface for $3 \mu \mathrm{m}$ long gold platelets exposed to $\lambda=800 \mathrm{~nm}$ light with oblique incidence $\left(\theta=60^{\circ}\right) \mathrm{TM}$ polarization for (a) $\lambda=625 \mathrm{~nm}$ and (b) $\lambda=1 \mu \mathrm{m}$. Contrast ranges of the micrographs are: $\{$ blue : red $\}=\left(\right.$ a) $\left\{9.8 \times 10^{-4}: 708\right\}$ and (b) $\left\{2 \times 10^{-3}: 264\right\}$.

Figure 34: Graph of the simulated time-averaged electric field squared at the gold surface of the rear tip vs. wavelength for a $3 \mu \mathrm{m}$ long gold platelet exposed to obliquely incident light $\left(\theta=60^{\circ}\right)$ for $\lambda=\{625 \mathrm{~nm}: 1 \mu \mathrm{m}\}$. Note: The fundamental wavelength for the PEEM's pulsed laser is marked.

Figure 35: (a) Simulation results of the time-averaged electric field squared at the triangular gold surface for $\lambda=800 \mathrm{~nm}$ light with oblique incidence $\left(\theta=60^{\circ}\right)$ and $\mathrm{TM}$ polarization; (b) PEEM micrograph (false color) with the same conditions. Contrast ranges of the micrographs are: $\{$ blue : red $\}=$ (a) $\left\{8.0 \times 10^{-5}: 149\right\}$ and (b) $\{1256: 40875\}$. Note: (b) is presented as the $4^{\text {th }}$ root of the photoemission yield. Original PEEM micrograph for (b) provided by Dr. Robert C. Word. 66

Figure 36: Simulation results of the time-averaged electric field squared for a triangular gold layer ( $35 \mathrm{~nm}$ below the gold surface, $15 \mathrm{~nm}$ above the ITO surface) for $\lambda=800 \mathrm{~nm}$ light with normal incidence $\left(\theta=0^{\circ}\right)$, TM polarization (a) and PEEM micrograph with the same conditions (b). Contrast range of the colored image (a) is:

$\{$ blue $:$ red $\}=\left\{6.8 \times 10^{-3}: 1572\right\}$. Note: The micrograph (b) is presented as the $3^{\text {rd }}$ root of the photoemission yield.

Figure 37: Plot showing the magnitude of the Poynting vector vs. vertical depth $(\mathrm{z})$ for the symmetric and anti-symmetric SPP modes for $\lambda=800 \mathrm{~nm}$ light obliquely incident $\left(\theta=60^{\circ}\right)$ for a vacuum-gold-ITO sample with $50 \mathrm{~nm}$ thick gold.

Plot courtesy of Dr. Robert C. Word. 


\section{Glossary of Terms, Abbreviations, and Symbols}

Element size (M): The maximum meshing size allowed within a region of the model.

Finite element analysis (FEA): The use of a finite element method for characterization.

Finite element method (FEM): A numerical method for solving problems with partialdifferential equations.

Focused Ion Beam (FIB): A charged particle beam system used for lithography and microscopy applications. Most commonly using a source of charged gallium ions.

Indium tin oxide (ITO): A widely used thin-film material, desirable for its electrical conductivity and optical transparency.

Meshing: The conversion of a solid object (2- or 3-dimensional) into an ordered polygonal or polyhedral grid. Necessary for computing finite element analysis.

Perfectly matched layer (PML): A type of boundary condition within a simulation model for propagating waves. Specifically, an absorbing exterior layer, which is designed so that incident waves do not reflect at the interface back into the model.

Photoelectric effect: The process by which shining light (photons) on a material produces the emission of electrons (i.e. photoelectrons) from the material.

Photoemission electron microscopy (PEEM): An imaging technique based on capturing electrons photoemitted from a material and converting them into an image via electron optics, a phosphor screen, and a CCD camera.

Photoemission yield ( $\mathrm{Y}_{\mathrm{PE}}$ ): A measure of the number of photoelectrons emitted from a material via the photoelectric effect from a specified number of photons.

Photoemitter: A material which can produce photoelectrons via the photoelectric effect. 
Plasmons: Collective oscillations of the charge density of a material [1].

Plasmonics: The creation, utilization, and manipulation of plasmons; analogous to electronics with electrons and photonics with photons.

Propagation Length (L): The distance for a surface plasmon polariton's energy to diminish by $1 / e(\sim 37 \%)$. This can be calculated if the indices of refraction of the materials and the wavelength of the incident excitation light are known.

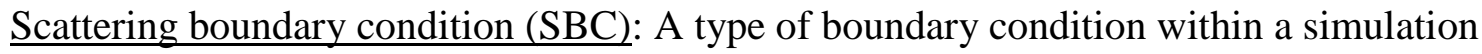
model for propagating waves. The boundary is perfectly-absorbing for perpendicularly incident waves, but only partially-absorbing for all other angles.

Surface plasmons: Non-localized plasmons which travel across the surface of a conductor.

Surface plasmon polaritons (SPPs): Electromagnetic waves, which typically travel along a metal-dielectric interface. SPPs are comprised of surface plasmons in the conductor coupled with photons in the dielectric.

Surface plasmon resonance (SPR): Resonant oscillation of conduction electrons at the interface between a dielectric and a conductor stimulated by light incident to the interface. SPR forms the basis of a variety of microscopy and sensing techniques.

Transverse electric (TE): A mode for a transverse electromagnetic wave such that there is no electric field in the plane of incidence.

Transverse magnetic (TM): A mode for a transverse electromagnetic wave such that there is no magnetic field in the plane of incidence. 
Basic terms and Constants (with symbols):

Angle of incidence $(\theta)$

Angle of polarization $(\varphi)$

Angular frequency $(\omega)$

Dielectric constant $(\epsilon)$

Electric displacement field $(\vec{D})$

Electric field $(\overrightarrow{\mathrm{E}})$

Electric permittivity $(\varepsilon)$

Electric permittivity in vacuum $\left(\varepsilon_{0}\right)$

Electric polarization $(\overrightarrow{\mathrm{P}})$

Energy (E)

Fast Fourier transform (FFT)

Frequency (f)

Index of refraction $(\mathrm{n}, \mathrm{k})$

Infrared (IR)

Kinetic energy (T)
Magnetic B-field $(\vec{B})$

Magnetic H-field $(\overrightarrow{\mathrm{H}})$

Magnetic magnetization $(\overrightarrow{\mathrm{M}})$

Magnetic permeability $(\mu)$

Magnetic permeability in vacuum $\left(\mu_{0}\right)$

Near-infrared (NIR)

Normal-vector (n)

Planck constant (h)

Propagation constant $(\beta)$

Speed (s)

Speed of light in vacuum (c)

Velocity (v)

Wavelength $(\lambda)$

Wavevector $(\mathrm{k})$

Wavevector of light in vacuum $\left(\mathrm{k}_{0}\right)$

Work function $(\Phi)$ 


\section{Introduction}

The field of plasmonics is well-established yet continues to be an area of great interest in science and technology. Advancements in fabrication methods and laser technology have enabled the development of micro- and nano-scale optical devices, which are increasingly of interest for electronic applications as modern silicon-based devices reach their peak performance. Plasmonic-based devices have been utilized in a wide range of applications, including: light localization [2], electron microscopy [3], phototherapy [4], sensors [5], heavy metal contamination [6], and plasmonic optics [7]. Reviews of the diverse areas using these devices can be found in the literature [8], [9].

Plasmonics is a comparatively new area, but the underlying theory and basic experimentation has been ongoing for nearly 70 years. Utilizing device configurations which can excite and propagate plasmons within a given material and structure, the movement of electromagnetic energy - in the form of surface plasmon polaritons (SPPs) — can be controlled down to the nanometer-scale. Plasmonic-based devices thus represent a next step in the miniaturization of light-based structures for the use of information transfer, localized electron sources, and many other applications. Theoretically, plasmons — collective oscillations of the charge density of a materialwere first proposed in 1952 by David Pines and David Bohm [10]. The properties of plasmons are direct consequences of Maxwell's Equations and can therefore be classically derived [11]. Surface plasmons - non-localized plasmons which can travel along the interface between a conductor and a dielectric — were first proposed in 1957 by 
Rufus Ritchie [12]. Throughout the subsequent 20 years, surface plasmons were experimentally characterized in detail, most significantly by T. Turbadar (1950s - '60s) and Heinz Raether, Erich Kretschmann, and A. Otto (1960s - '70s) [13]. In certain situations, surface plasmons - moving across the surface of a conductor-can couple with photons in the adjacent dielectric material and form electromagnetic waves known as surface plasmon polaritons (SPPs) [14]. The mathematical formulation describing these surface waves was previously derived in the late $19^{\text {th }}$ century by Arnold Sommerfeld for radio waves propagating across the surface of a conductor [15]. The work presented here will focus on the excitation of SPPs and the accompanying secondary response of plasmon-driven electron emission (i.e. photoemission) within specific optical structures.

Currently, gold-based devices are often used for their strong plasmonic responses due to their favorable optical properties. Single-crystalline gold micro-rods and triangular platelets — which may be synthesized or manufactured through various processes - have been of particular interest [16]-[18]. Recent research has investigated optical responses of both triangular platelets [19], [20] and structures manufactured from them: such as circular ring gratings [21], [22]. Typically, features are milled into the material using a gallium-based focused ion beam (FIB), but recent work pushes to smaller dimensions and higher repeatability through electron beam lithography [23]. To image these nano-scale optical effects, a variety of microscopy techniques have been employed, including: surface potential [24], electron energy loss [25], and photoemission electron microscopy (PEEM). As detailed in the next section, imaging plasmonic responses in gold using 
PEEM requires multi-photon excitation (nP-PEEM), which results in a non-linear process providing strong image contrast. Due to its unique capabilities, PEEM has been broadly applied to study surface plasmon propagation in metallic films and devices [26]-[28].

In this work, we use existing electromagnetic theory and computer simulation to explain and reproduce localized photoemission observed in triangular gold platelets with PEEM. A finite element method (FEM) based modeling technique is used to reproduce prior experimental results produced at PSU and to characterize the dependence of the observed photoemission's rate and location on the wavelength and polarization of the PEEM's incident laser. The spatial resolution of our simulation and the resolution of photoelectron microscopy are on a scale smaller than the wavelength of the light and the single-crystalline gold structures are demonstrated to behave as planar patch antennas. 


\section{Photoemission Electron Microscopy (PEEM) for Plasmonics}

\subsection{Background Theory and PEEM Overview}

Since its theoretical formulation by Albert Einstein in 1905 and its experimental confirmation in 1914 by Robert Millikan, the photoelectric effect has been broadly applied in optics for the past 100 years and is the foundation on which photoemission electron microscopy is built. In the process, shining light of a sufficiently high frequency on a material will cause the emission of electrons, referred to as photo-electrons or photoemitted electrons. According to the Planck - Einstein relationship,

$$
E=h f
$$

where $\mathrm{E}$ is the photon energy, $\mathrm{h}$ is the Planck constant, and $\mathrm{f}$ is the frequency of the photon. With a definition of the energy of a photon, Einstein then expressed the emission of an electron from a material as:

$$
T_{\max }=E-\Phi=(h f)-\Phi
$$

where $T_{\max }$ is the maximum photoelectron energy and $\Phi$ is the work function of the material. Thus, if the incident photon has energy higher than the work function of the material, an electron can be emitted and will have a maximum kinetic energy equal to the difference.

In practice, the above process does not have to be exactly one photon exciting one electron. If multiple photons interact with a bound electron within a short enough time interval, it is possible their combined energies can excite the electron from the material. This processed is named multi-photon electron emission and can technically occur for any number of photons which combined have a sufficiently higher energy than the work 
function of the material. As expected, the larger the number of photons required for the process, the less probable the event will occur. As an example, let us consider a gold surface with a work function of $5.1 \mathrm{eV}$. At $400 \mathrm{~nm}$,

$$
\begin{aligned}
& \mathrm{E}=\mathrm{hf}=\mathrm{h}\left(\frac{\mathrm{c}}{\lambda}\right) \\
& =\frac{\left(4.14 \times 10^{-15} \mathrm{eV} \cdot \mathrm{s}\right)\left(2.99 \times 10^{8} \mathrm{~m} / \mathrm{s}\right)}{\left(400 \times 10^{-9} \mathrm{~m}\right)}=3.7 \mathrm{eV}
\end{aligned}
$$

Thus, for $\mathrm{E}=3.7 \mathrm{eV}$ and $\Phi=5.1 \mathrm{eV}$ it would require at least two photons to excite an electron from the material (2p). For $\lambda=700 \mathrm{~nm}, \mathrm{E}=2.13 \mathrm{eV}$ and a minimum of 3 photons are required $(3 p)$.

Although the photoelectric effect at the atomic level is inherently a quantum mechanical process, the work here involves the collective effect from many photons interacting with many electrons. In the experiments, materials with high-electron densities are used and the available supply of electrons in the material is far greater than the relatively small number of photoelectrons created through the photoelectric effect; as it is an inefficient process at best. As we are considering the effects of large groups of particles, instead of individual photons or electrons, a classical treatment of their behavior is a reasonable approximation for the results observed in PEEM. Thus, although PEEM is based on a quantum mechanical process, the net quantum effects are relatively small and the overall optical responses from the materials can be explained and approximated with classical electromagnetic theory. 
In PEEM, electrons emitted from a sample surface are collected and used to provide an enlarged image on a phosphor screen. This image is then captured by a CCD camera (Figure 1, below). Collecting and controlling the electrons emitted from the sample is a multistage process, where the electrons are passed through a series of electronic lenses, deflectors, and apertures. In addition, an aberration-corrected mirror is used to improve the resolution of the microscope. As the image is produced via electron optics (in place of traditional light-based optics), the resolution of the microscope is not restricted by the diffraction limit of the laser used to emit the electrons. This allows the resolution of PEEM to push beyond the traditional resolution limit of light based microscopy [29].

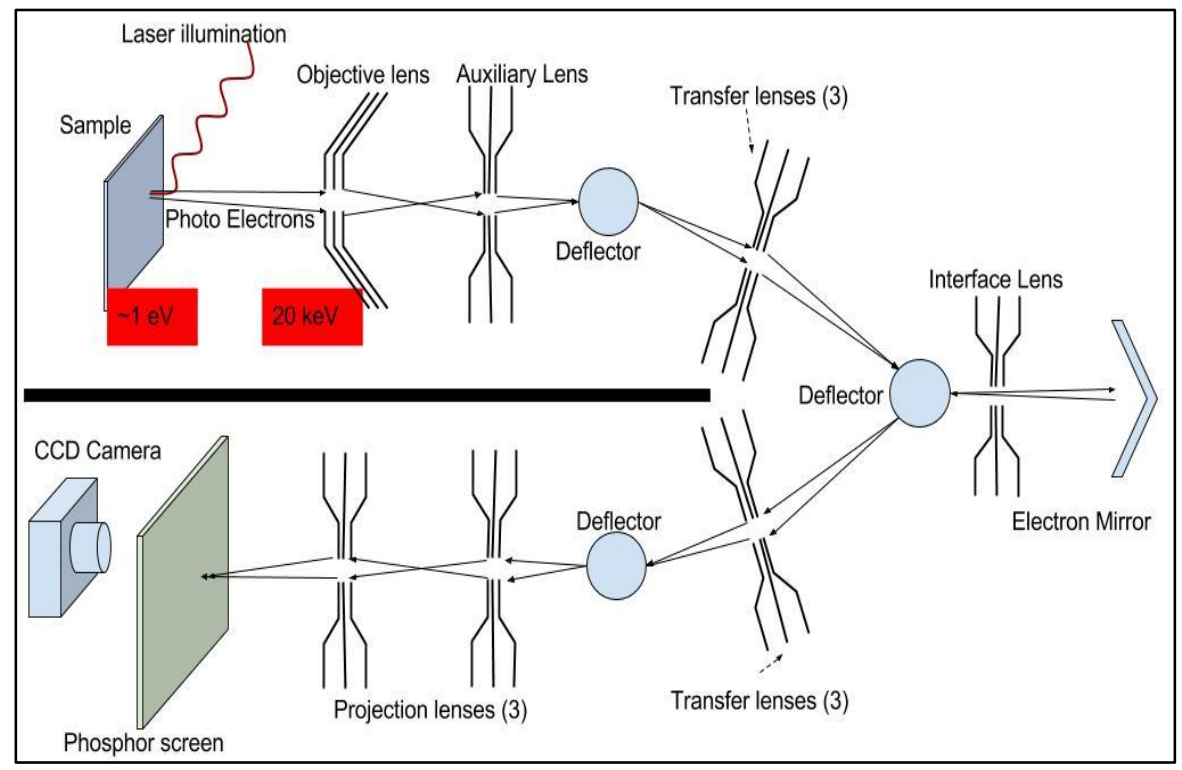

Figure 1: Illustration of example PEEM optics (simplified), detailing the laser illumination, sample, lenses, deflectors, phosphor screen, and CCD camera.

- Reprinted with permission from Photoemission Electron Microscopy for Analysis of Dielectric Structures and the Goos-Hänchen Shift, by Theodore Axel Stenmark, Portland State University, 2016 [30]. 
Multiphoton PEEM (nP-PEEM) — a non-linear imaging process utilizing multiphoton electron emission — can be used in place of traditional single-photon PEEM imaging. The energy of the incident light can be varied within the fundamental frequency range of the laser and to higher-order harmonics, enabling the observation of a widerange of materials and optical effects. In general, a laser needs to be used due to the strong coherence and high intensity of its emitted photons. Coherence is achieved through a beam-splitting process and is therefore independent of the coherence of the laser source itself. On the other hand, the intensity of the source is critical as only a large illumination intensity will lead to sufficient photoemission yields from the sample. Due to the non-linearity of the multi-photon emission process, a modest increase in the source intensity could produce a significant increase in photoemission from the sample. The work function of most interesting materials prohibits the use of 1P-PEEM for the visible and IR ranges, but the high intensities of ultrafast pulsed lasers can be enough to overcome these energy barriers and enable multi-photon PEEM imaging [30].

In addition to the necessity for nP-PEEM for certain materials and wavelength ranges, the non-linear response of multi-photon excitation provides strong image contrast through the imaging technique's sensitivity to variations in the local photon density at the surface of the sample. Image contrast in general in PEEM arises from the spatial distribution of photoemitted electrons from the sample. Most of the emitted electrons are from the top $\sim 5-10 \mathrm{~nm}$ of the sample, which results in a strong surface imaging technique. The main image contrast mechanisms include: (1) material and (2) geometrical differences in the sample and local variations in the (3) electron or (4) the photon density. 
The material composition of a sample and differences in its geometry can both affect the yield of emitted photoelectrons. The images in this work deal with flat and homogeneous surfaces, which will result in minimal image contrast from material differences within the area of interest (single-crystalline material). Instead, the contrast arises from variations in the light intensity across the surface, which is due to diffraction effects.

The photoemission yield $\left(\mathrm{Y}_{\mathrm{PE}}\right)$ (i.e. the yield of electrons emitted from the material) is proportional to the intensity of the light (i.e. the number of photons). In a classical treatment - as is considered by the FEM simulations - the intensity is proportional to the square of the electric field. Thus, we can relate the two quantities by the following:

$$
\begin{aligned}
& Y_{P E} \propto|I| \quad \text { and } \quad I \propto|\vec{E}|^{2} \\
& \rightarrow Y_{P E} \propto|\vec{E}|^{2}
\end{aligned}
$$

For a multi-photon process, we must also consider the number of photons required per emitted electron and our relationship becomes:

$$
\begin{aligned}
& Y_{P E} \propto\left(|\vec{E}|^{2}\right)^{n} \\
& \rightarrow Y_{P E} \propto|\vec{E}|^{2 n}
\end{aligned}
$$

where $\mathrm{n}$ is the number of photons required per photoelectron emission [29]. The larger the number of photons required for an emission (i.e. $n$ ), the greater the potential contrast difference within the image, but the lower the signal strength as the photoemission yield is inversely proportional to the transition probability (i. e. $\mathrm{Y}_{\mathrm{PE}} \propto 1 /$ transition prob.). 


\subsection{Experiment and Sample Details}

Our lab has a home-built aberration-corrected PEEM system, which has a best demonstrated resolution of $5 \mathrm{~nm}$ [31]. The microscope utilizes a Spectra-Physics Ti:Sapphire laser (Figure 2, below) with $1 \mathrm{~W}$ average output power, 60 fs pulse width, and $80 \mathrm{MHz}$ repetition rate. The fundamental wavelength of the laser is in the range of $\lambda=780-920 \mathrm{~nm}$. Second, third, and fourth harmonic generators are available to obtain wavelengths beyond the fundamental. A tunable waveplate allows for linear polarization across the entire spectrum of $\varphi=\left\{0: 360^{\circ}\right\}$. Sample illumination can be achieved at an oblique angle of $\theta=60^{\circ}$ to the surface normal from above or at normal incidence $\left(\theta=0^{\circ}\right)$ from below (Figure 3, below). In addition, a continuous-wave, frequency-doubled Ar-ion

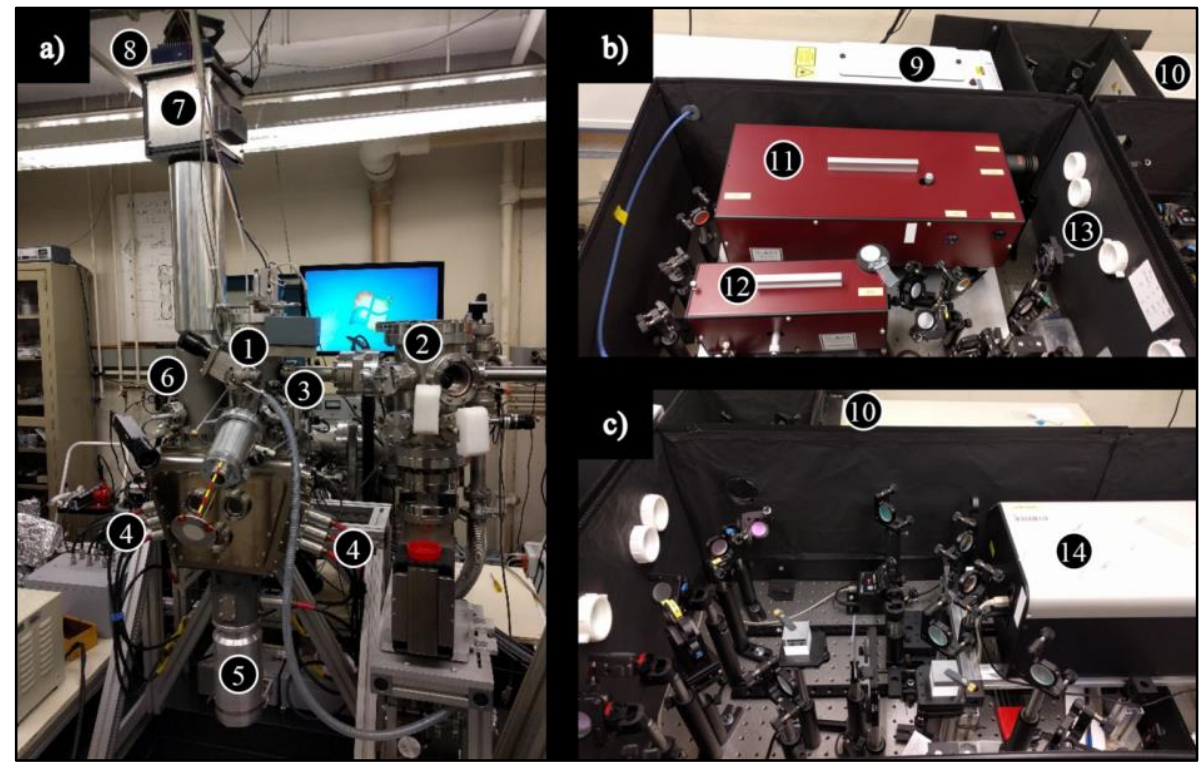

Figure 2: Photographs of the PEEM components (a) and the laser system (b, c). Labeled components: (1) sample loadlock, (2) sample preparation chamber, (3) objective lens, (4) transfer lenses, (5) diode electron mirror, (6) projection lenses; (7) phosphor screen, (8) CCD camera, (9) Ar-ion CW laser, (10) Mai Tai Ti-Sapphire pulsed laser, (11) second / third harmonics generator, (12) fourth harmonic generator, (13) tunable waveplate, and (14) spatial modulator. 
laser with $\lambda=244 \mathrm{~nm}$ is available - primarily for alignment of the microscope - and a non-coherent mercury lamp with light emission in the $170-250 \mathrm{~nm}$ range may be used.

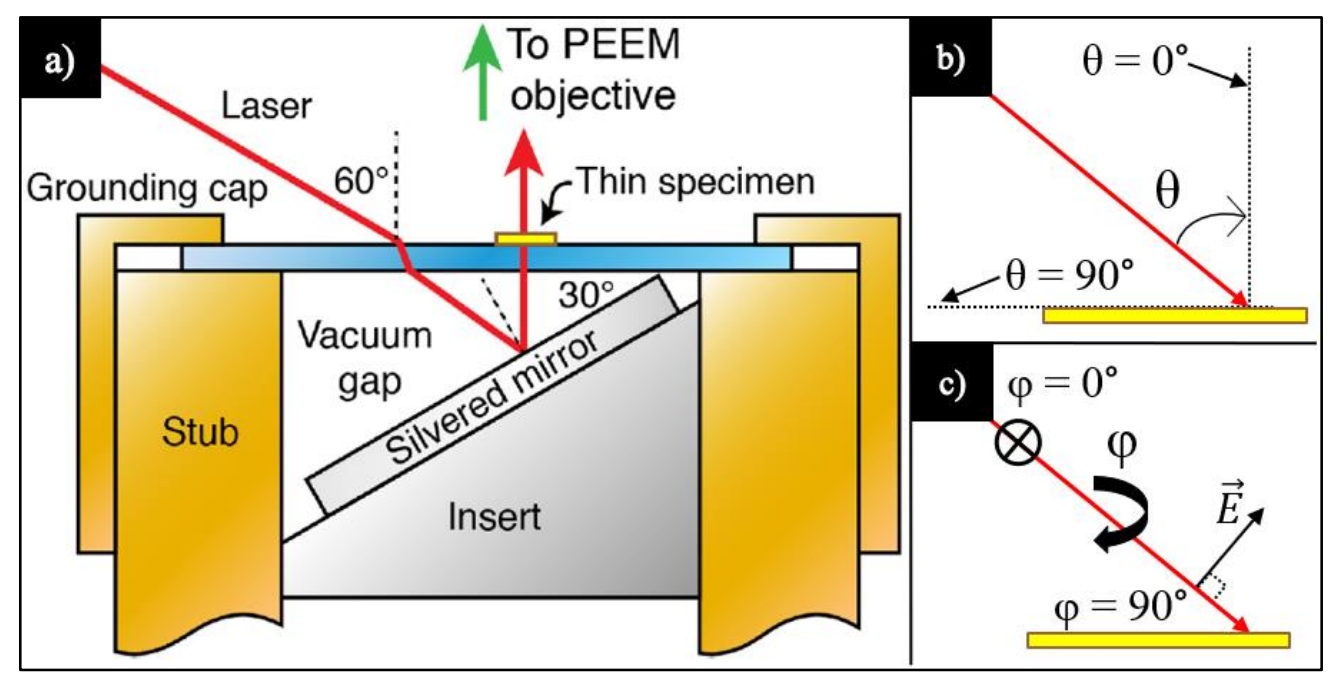

Figure 3: Illustrations of the: (a) PEEM specimen stub, depicting oblique (from above) and normal (below) incidence [26]; (b) incidence angle $\theta$; and (c) polarization angle $\varphi$.

- Image (a) reprinted with permission from Photonic and plasmonic surface field distributions characterized with normal- and oblique-incidence multi-photon PEEM, by R.C. Word and R. Könenkamp, Ultramicroscopy, vol. 183, pp. 1139-1351, 2017.

Our samples consists of thin, triangular gold platelets deposited onto a simple planar stack (Figure 4, below). The bulk substrate was purchased from SPI Supplies Inc. and has a $275 \pm 15 \mathrm{~nm}$ thick indium tin oxide (ITO) layer coated onto a $0.2 \mathrm{~mm}$ thick borosilicate glass coverslip [32]. The gold platelets are grown in a wet-chemical technique (Guo et al) and are single-crystalline with a $<111>$ surface orientation. The triangles are equilateral with the majority measuring $\sim 3-5 \mu \mathrm{m}$ in length and have thicknesses $\sim 50 \mathrm{~nm}$. At extremes, the triangles have side lengths between $\sim 1-15$ $\mu \mathrm{m}[16]$. ITO is transparent for the visible spectrum and weakly absorbing in the near- 
infrared (NIR) and infrared (IR) ranges. The gold platelets conversely were previously shown to have increasing absorption in the NIR range [16].

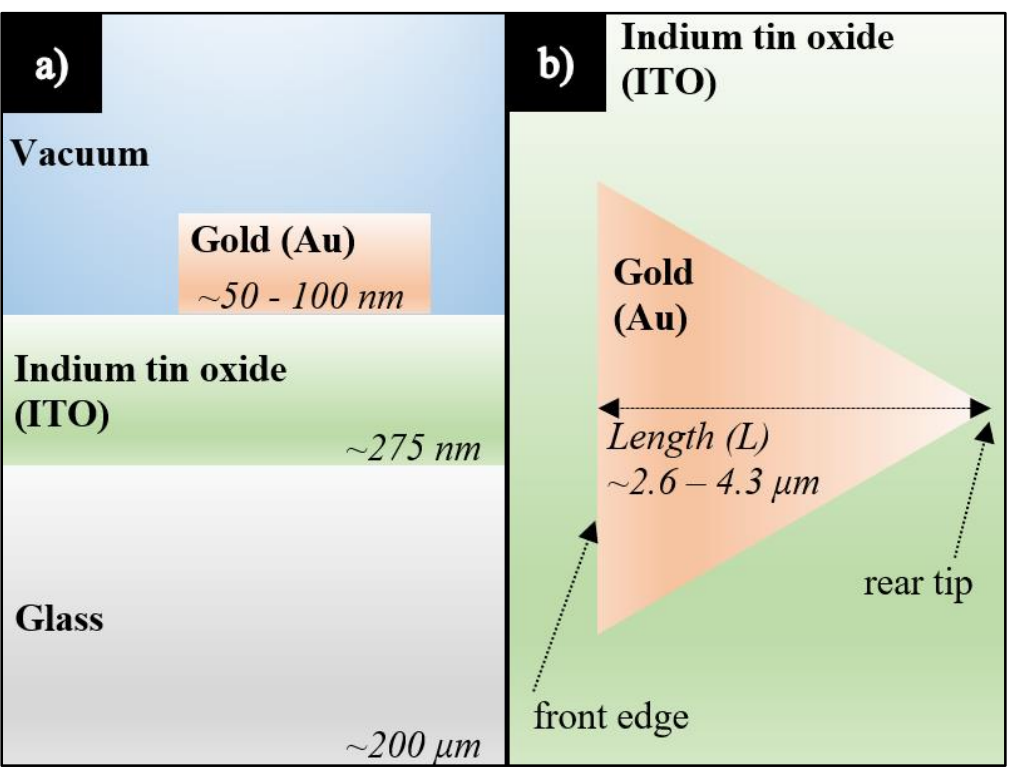

Figure 4: Illustration of an example gold (Au)-ITO-glass sample from (a) cross-sectional and (b) top-down views.

For the experiments, separate samples were illuminated obliquely from above $\left(\theta=60^{\circ}\right)$ or normally from below $\left(\theta=0^{\circ}\right)$, both with $\lambda=800 \mathrm{~nm}$ light with a beam diameter of $\sim 100 \mu \mathrm{m}$ (Figure 3, above). Considering gold has a work function of $5.1 \mathrm{eV}$, ITO of $4.5 \mathrm{eV}$, and at $\lambda=800 \mathrm{~nm}$ photons have an energy of $1.55 \mathrm{eV}$, the PEEM micrographs will involve predominantly 3- and 4-photon photoemission processes [26]. Under these conditions, the best resolution of the PEEM system is $\sim 20 \mathrm{~nm}$. 


\section{Finite Element Method (FEM) Based Modeling}

\subsection{Method Overview}

Algorithms and techniques for solving differential equations have existed since the mathematics were first formulated. Currently, the dominant approach for solving partial-differential equations (PDEs) is the finite element method (FEM), which approximates solutions to the equations by replacing them with difference equations, where the finite differences relate to the derivatives. In this manner, a solution is approximated through discretization of the original equations [33]. For time-dependent PDEs, time-dependent methods were established and have been used in computational fluid dynamic problems since the 1950s [34]. Using FEM based techniques is referred to in shorthand as finite element analysis (FEA). As a broad overview, FEA involves converting a series of PDEs into a system of algebraic equations. To solve the large system of equations, it then splits the system into simpler components referred to as "finite elements" [35]. A larger system of equations that represent these finite elements is then amassed to represent the overall problem. Lastly, FEA implements methods from the calculus of variation to form an approximate solution to the original problem by minimizing an accompanying error function [35].

Using COMSOL Multiphysics — a commercially available licensed software suite-laser illumination of a sample, with defined constituent materials can be simulated to match PEEM experiments. Creating 2- or 3-dimensional geometric models, each object or layer can be assigned a material type, which will include its specified optical properties (Figure 5, below). By establishing a meshing style, these objects will be 
converted to a grid of connecting shapes - typically polygons (2D) or polyhedrons (3D) — which can have minimum and maximum sizes associated with them. Finally, setting up an incident or stationary light source (e.g. an electromagnetic plane-wave) and appropriate boundary conditions, the software will approximate solutions to Maxwell's Equations for the time-averaged electromagnetic fields at each point within the model.

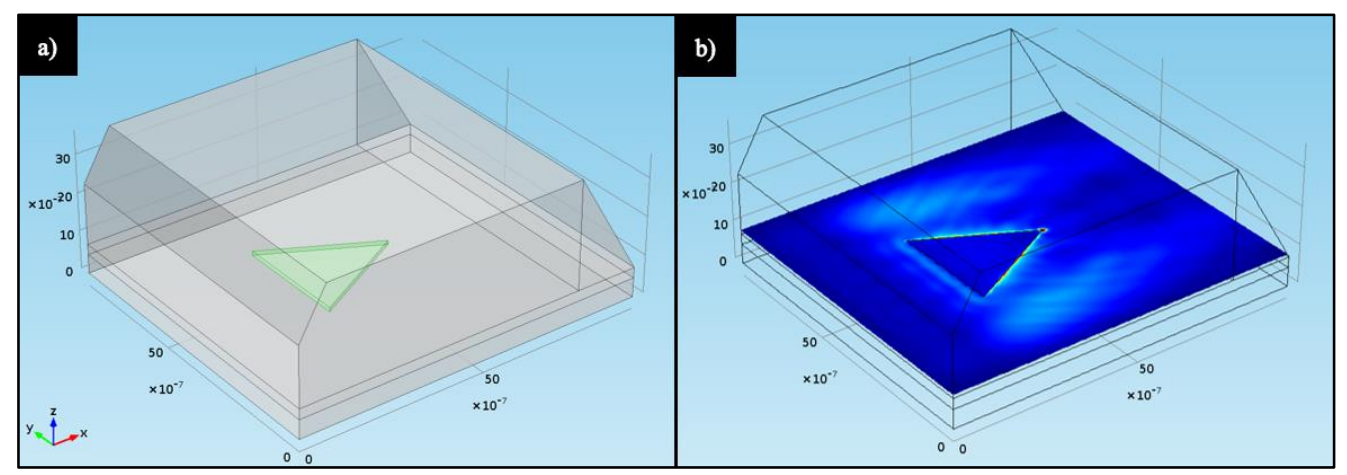

Figure 5: 3D COMSOL model of a gold-ITO-glass sample with a surrounding vacuum region: (a) gold region selected in green and (b) simulation results of the time-averaged electric field at the gold surface for $\lambda=800 \mathrm{~nm}$ obliquely $\left(\theta=60^{\circ}\right)$ incident light.

Our simulation results will only consider linear effects yet can be comparable to experimental PEEM micrographs if we set both data sources in terms of the electric field squared $|\vec{E}|^{2}$. Recall, micrographs obtained from PEEM display the time-averaged photoemission yield (Y $\mathrm{PE}$ ), while simulation results will produce the electric field $|\vec{E}|$ and that these quantities are directly proportional as $Y_{\mathrm{PE}} \propto|\overrightarrow{\mathrm{E}}|^{2 n}$, where $\mathrm{n}$ is the number of photons required for a single photoemission event. If we consider the $|\vec{E}|^{2}$ for simulation results and use the $\mathrm{n}$-th root of the experimental photoemission yield for the contrast scale for PEEM micrographs, we can directly compare the two results. 


\subsection{Parameter Details}

All material layers in the models have sharp square edges instead of more realistic curved edges (Figure 6.a, below). This was initially performed in 2D for simplicity but continued in 3D models due to COMSOL errors with combining and meshing discrete objects curved in all three dimensions. To verify this geometric simplification would still provide a reasonable approximation, a study of the electric field response from curved and square edges was completed in 2D. The electric field results showed only minor differences between square and curved edges, with the overall responses remaining the

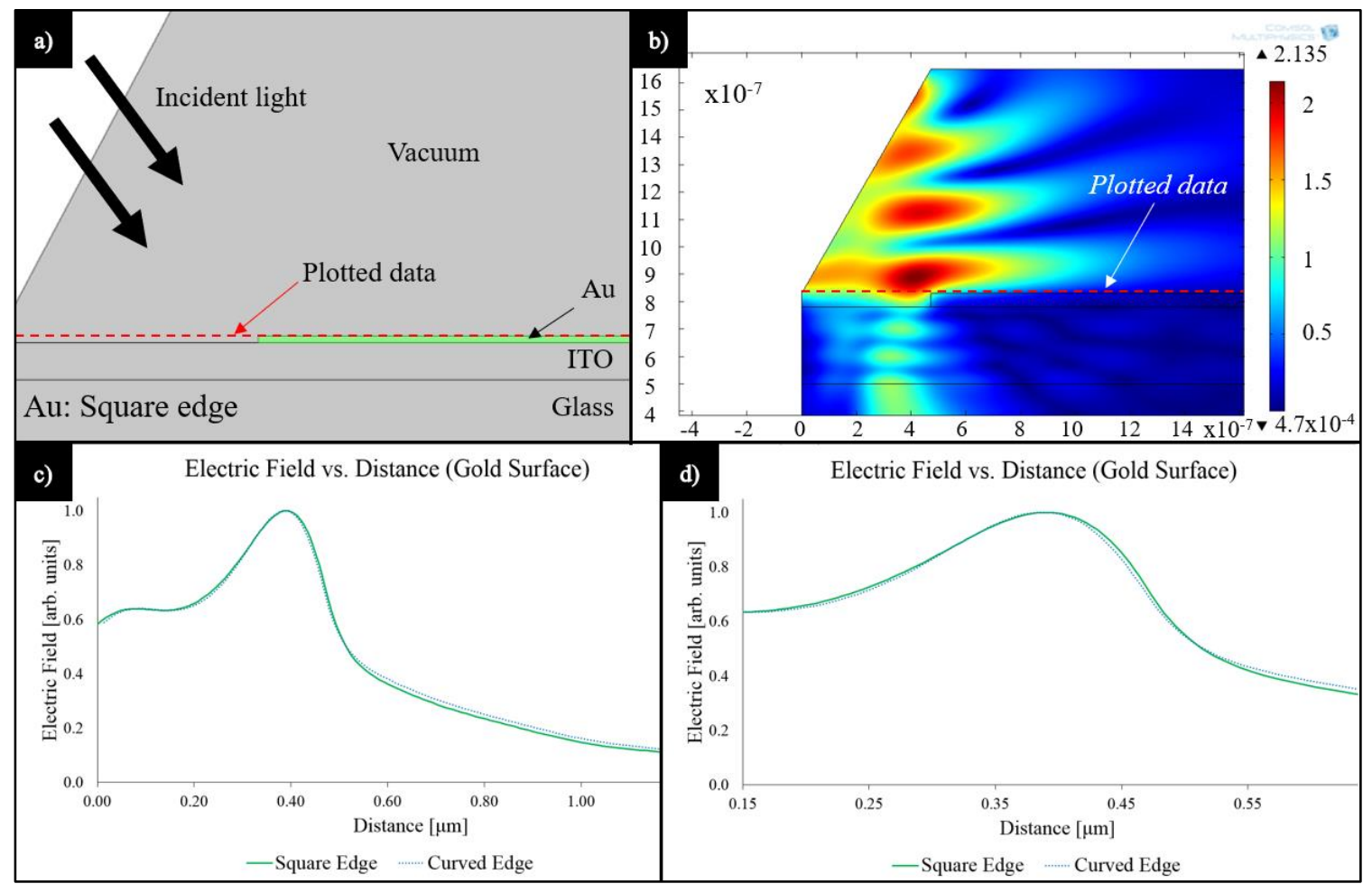

Figure 6: (a) Cross-sectional view of a 2D COMSOL model of a gold-ITO-glass sample with the layers labeled and the gold region highlighted in green; (b) simulation results of the normalized electric field for $\lambda=800 \mathrm{~nm}$ light incident obliquely from above at $\theta=60^{\circ}$ to the surface normal with TM polarization; (c) graph of the simulated electric field vs. distance from the simulation boundary for gold regions with a square (green, solid) and curved (blue, dotted) front edge; (d) magnified view of graph (c). 
same (Figure 6.c, d; above). By applying a minor linear translation in the $\mathrm{x}$ - and $\mathrm{y}$-axis to one of the data sets, the results line up even closer. Specifically, a linear translation was applied to the curved edge results with $\mathrm{x}_{\text {shift }}=14 \mathrm{~nm}$ and $\mathrm{y}_{\text {shift }}=0.008$.

The thicknesses of the sample layers increase down the stack: gold $(50 \mathrm{~nm})$, ITO $(280 \mathrm{~nm})$, and glass $(500 \mathrm{~nm})$. The vacuum layer above the sample is $2.2 \mu \mathrm{m}$ thick at its maximum. The gold triangular platelets are equilateral and measure $3 \mu \mathrm{m}$ in length for most of the work, with an associated width of $\sim 3.5 \mu \mathrm{m}$. The edge of the platelet is offset from the simulation boundary by $2.0 \mu \mathrm{m}$ on the front, left, and right; and by $\sim 4.8 \mu \mathrm{m}$ on the rear (Figure 5, above). The meshing of the model is set smallest for the gold layer, as this is the primary area of interest. For 2D simulations, a triangular meshing with a maximum element size (M) of $10 \mathrm{~nm}$ is used for the gold region. For 3D simulations, a tetrahedral meshing with $\mathrm{M}=50 \mathrm{~nm}$ is used for the gold region and the surrounding $25 \mathrm{~nm}$ (in all directions). This extension of the smallest maximum meshing size avoids having both a material and meshing size transition at the area of interest along the surfaces of the gold layer. For both 2- and 3D simulations, the remainder of the model (i.e. ITO, glass, vacuum) has $\mathrm{M}$ tied to the wavelength of the light $(\lambda)$, the index of refraction of the material (n), and a meshing coefficient $(c=5)$ such that $M=\lambda / 5 n$.

The incident laser light is modeled using the plane wave approximation. The direction of propagation of the incident light is perpendicular to a slanted edge in the upper-left region of the model (Figure 5, above) and has components $\left\{\mathrm{k}_{\mathrm{x}}, \mathrm{k}_{\mathrm{y}}, \mathrm{k}_{\mathrm{z}}\right\}=\mathrm{k}_{0} \cdot\{\sin (\theta), 0,-\cos (\theta)\}$, where $\mathrm{k}_{0}$ is the wavevector of the incident light in vacuum and $\theta$ is the angle of incidence relative to the surface normal. For our 


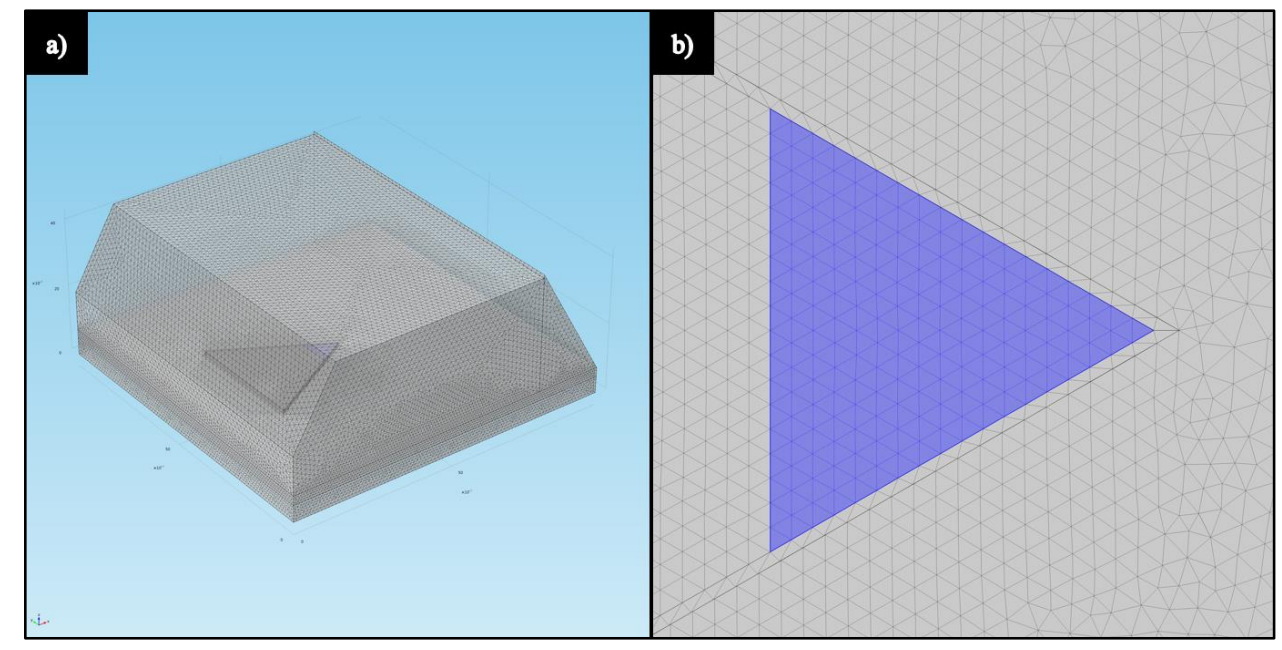

Figure 7: 3D COMSOL model of a gold-ITO-glass sample with a surrounding vacuum region with the tetrahedral meshing displayed: (a) entire model and (b) magnified view of the tip region of the triangular gold layer (highlighted in purple).

experiments, $\theta=60^{\circ}$ or $0^{\circ}$ to match the oblique and normal PEEM illuminations. The electric field is perpendicular to the propagation direction of the light and is dependent on both the incident angle $(\theta)$ and the polarization angle $(\varphi)$ with components $\left\{E_{x}, E_{y}, E_{z}\right\}=|\vec{E}| \cdot\{\cos (\theta) \cdot \sin (\varphi),-\cos (\varphi), \sin (\theta) \cdot \sin (\varphi)\}$, where $|\vec{E}|$ is the magnitude of the electric field. The model uses an approximation of the full Sommerfeld radiation condition at its boundaries, namely a first-order scattering boundary condition (SBC), expressed by:

$$
\boldsymbol{n} \cdot\left(\nabla E_{z}\right)+i k_{0} E_{z}=0
$$

where $\mathbf{n}$ is the normal-vector [36]. As can be seen in Figure 8 (below), a wave which is incident directly perpendicular to the boundary will be fully absorbed, while all other incidences will result in partial, increasingly small reflections. Thus, these boundary 
conditions ensure that reflections occurring at the boundaries of the model remain small and will only negligibly affect the simulation results.

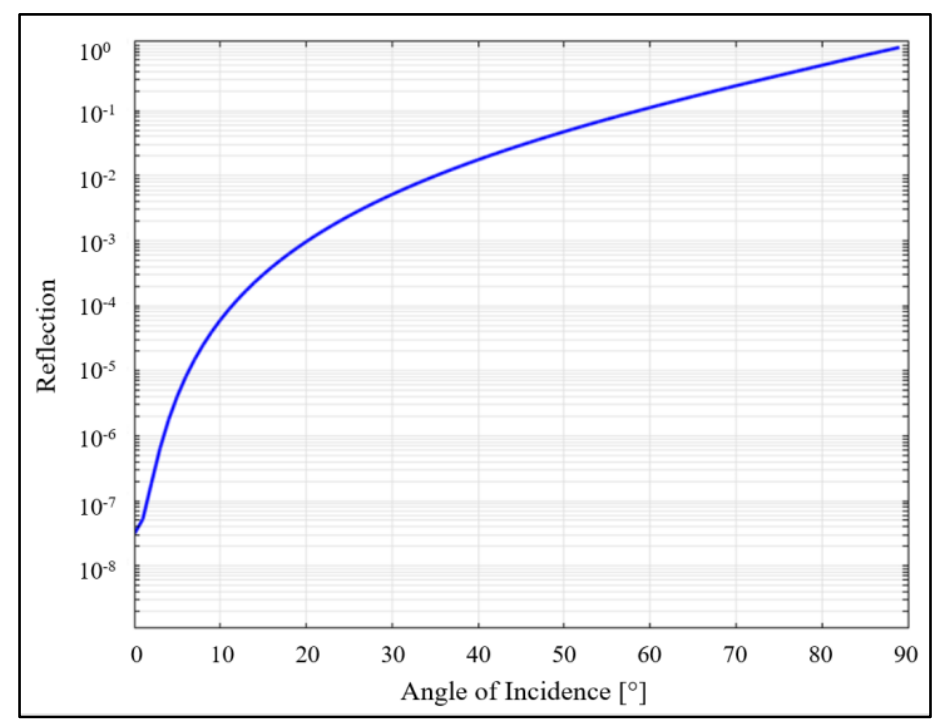

Figure 8: Graph of the reflection of a plane wave vs. incidence angle for a $1^{\text {st }}$ order SBC.

- Adapted from Using Perfectly Matched Layers and Scattering Boundary Conditions for Wave Electromagnetics Problems, by Walter Frei, COMSOL Blog, 2015 [36].

To evaluate the polarization dependence, the model is simulated for the entire polarization range of $\varphi=\left\{0: 360^{\circ}\right\}$. For wavelength dependence, the incident light is varied in the range of $\lambda=\{625 \mathrm{~nm}: 1 \mu \mathrm{m}\}$. A summary of the major simulation parameters evaluated throughout the development of the 2D and 3D sample models is provided in Table 1 (below).

\begin{tabular}{|l|c|c|c|}
\hline Simulation Parameter & Value & Range Evaluated & Increment \\
\hline Front edge distance & $2 \mu \mathrm{m}$ & $0-4 \mu \mathrm{m}$ & $1 \mu \mathrm{m}$ \\
\hline Side edge distance & $2 \mu \mathrm{m}$ & $0.4-2 \mu \mathrm{m}$ & $0.4 \mu \mathrm{m}$ \\
\hline Surrounding mesh distance & $25 \mathrm{~nm}$ & $0-100 \mathrm{~nm}$ & $25 \mathrm{~nm}$ \\
\hline Meshing coefficient (c) value & 5 & $4-8$ & 1 \\
\hline Maximum meshing size & $50 \mathrm{~nm}$ & $50-135 \mathrm{~nm}$ & ------------ \\
\hline Gold layer thickness & $50 \mathrm{~nm}, 1 \mu \mathrm{m}$ & $50-100 \mathrm{~nm}, 1 \mu \mathrm{m}$ & ------------ \\
\hline
\end{tabular}

Table 1: Major parameters evaluated for the 3D COMSOL gold-ITO-glass model. 
Lastly, the values for the refractive indices $(n, k)$ were found in literature for the various materials: gold [37], ITO [38], and glass [39]. The model also includes a vacuum region above the gold / ITO layers, with values fixed at $(1.0,0.001)$ for all wavelengths.

\begin{tabular}{|l|c|c|}
\hline Material & [Real] Refractive index (n) & Extinction coefficient (k) \\
\hline Vacuum & 1.0 & 0.001 \\
\hline Glass & 1.45 & 0 \\
\hline Gold & 0.1535 & 4.908 \\
\hline Indium tin oxide (ITO) & 1.77 & 0.0049 \\
\hline
\end{tabular}

Table 2: Index of refraction values for $\lambda=800 \mathrm{~nm}$.

- Optical data obtained from the following sources:

Glass: Interspecimen comparison of the refractive index of fused silica, by I. H. Matlitson, J. Opt. Soc. Am., vol. 55, pp. 1205-1208, 1965 [39].

Gold: Optical constants of the noble metals, by P.B. Johnson and R.W. Christy, Phys. Rev. B, vol. 6, no. 12, pp. 4370-4379, 1972 [37].

ITO: Subnanometer-accuracy optical distance ruler based on fluorescence quenching by transparent conductors, by R.J. Moerland and J.P. Hoogenboom, Optica, vol. 3, pp. 112-117, 2016, [38]. 


\subsection{Model Improvements}

A variety of improvements to the model have been made to address specific issues or concerns beyond a basic implementation. A few of them are listed below:

1. Additional space was included beyond the rear tip of the triangular gold region, which when combined with an angled rear-boundary minimizes light reflection back into the simulation (i.e. edge of the model is angled to be perpendicular to the light reflected directly off the gold and ITO surfaces to maximize absorption by the boundary). For the results presented, there was an additional $2.84 \mu \mathrm{m}$; for a total of $4.84 \mu \mathrm{m}$ from the rear tip of the gold layer to the exterior edge of the angled boundary.

2. The layers surrounding the gold region were artificially split into multiple regions, such that the tightly spaced meshing of the gold region could be extended by an adjustable distance in all directions. For the results presented, this was set to $25 \mathrm{~nm}$. This reduced artificially introduced effects from transitioning the meshing size at the boundary of two different materials, especially at the area of interest.

3. The gold layer for the triangular models was separated into two meshing regions, with adjustable relative lengths. This allows the meshing in the rear tip (i.e. main area of interest) to be set with a smaller maximum meshing size (M) than the rest of the "bulk" gold layer. This is only needed for relatively large triangles ( $\mathrm{L}>5 \mu \mathrm{m}$ ).

4. The indices of refraction for the various materials are wavelength dependent (except for vacuum), so reference tables were created to automatically adjust the material properties of the model for a large range of wavelengths $(\lambda=400 \mathrm{~nm}: 1 \mu \mathrm{m})$. 
Additional improvements were desired, but not successfully implemented with the allotted resources due to unresolved software errors. These include:

A. Implementing all curved edges for the triangular gold platelet region. Although not difficult conceptually, continued issues with COMSOL hampered this improvement.

B. Switching from a scattering boundary condition (SBC) to a perfectly matched layer (PML) boundary condition. PML boundary conditions are needed to completely suppress reflections at the boundary of the model. Attempts to convert previously developed models (by other group members) with PML boundaries were unsuccessful and this was not a high enough priority for completion, although technically it should provide more accurate approximations. There are also memory limitation concerns considering the scales of the experiments to reproduce, since a PML requires significantly more processing power than a SBC. 


\section{Surface Plasmon Polaritons (SPPs)}

\subsection{Background Theory}

In place of reproducing a full derivation of the underlying theory of surface plasmon polaritons (SPPs) — which can be found in the literature [15], [40], [41]—we will highlight the key results which should be considered for the experiments presented in this work. This section predominately references the derivation and results from Dr. Stefan A. Maier's text, "Plasmonics: Fundamentals and Applications," but similar derivations exist from many authors.

To begin, recall that SPPs are electromagnetic surface waves - traveling along the interface between a conductor and a dielectric - coupled to oscillations of the electron density within the conductor. These electromagnetic surface waves typically occur at the interface between a conductor and a dielectric, although under specific circumstances they may occur between two conducting surfaces. The amplitude of SPPs will decay exponentially with the perpendicular distance to the surface and in this regard, SPPs are highly confined to the surface of the conductor-dielectric interface. SPPs are a classical effect and their wave and field equations can be derived directly by applying Maxwell's equations for electromagnetism in media to the interface between a conducting and dielectric surface. Recall, Maxwell's equations for media (in SI units):
Gauss' Law:
$\vec{\nabla} \cdot \vec{D}=\rho_{\text {ext }}$
Gauss' Law for Magnetism:
$\vec{\nabla} \cdot \vec{B}=0$
Faraday's Law of Induction: $\quad \vec{\nabla} \times \vec{E}=-\frac{\partial \vec{B}}{\partial t}$ 
Ampere's Circuital Law: $\quad \vec{\nabla} \times \vec{H}=\vec{J}_{\text {ext }}+\frac{\partial \vec{D}}{\partial t}$

where $\rho_{\text {ext }}$ and $\vec{J}_{\text {ext }}$ are the external charge and current densities. The four fields $(\vec{E}, \vec{D}, \vec{B}$, and $\vec{H})$ are additionally connected via the electric polarization $(\vec{P})$ and magnetic magnetization $(\overrightarrow{\mathrm{M}})$ :

$$
\begin{aligned}
& \vec{D}=\varepsilon_{0} \vec{E}+\vec{P} \\
& \vec{H}=\frac{1}{\mu_{0}} \vec{B}-\vec{M}
\end{aligned}
$$

where $\varepsilon_{0}$ is the electric permittivity in vacuum and $\mu_{0}$ is the magnetic permeability in vacuum. For this application, we will consider non-magnetic materials and thus there will be no magnetization. In addition, we limit ourselves to linear, isotropic media.

The further simplified relationships are thus:

$$
\begin{aligned}
& \vec{D}=\varepsilon \vec{E} \\
& \vec{B}=\mu \vec{H}
\end{aligned}
$$

where $\varepsilon$ is the relative permittivity and $\mu$ is the relative permeability.

The work presented here is time-independent—due to the inherent time-averaging in PEEM imaging - thus, to evaluate the expected wave behavior we will want the Helmholtz equation (i.e. the harmonic time dependence of the wave equation). To relate $\vec{E}$ in terms of propagating, bound surface waves we will express $\vec{E}$ as a basic timedependent harmonic oscillator:

$$
\vec{E}(\vec{r}, t)=\vec{E}(\vec{r}) e^{-i \omega t}
$$


where $\omega$ is the angular frequency of the oscillation. From the two Maxwell curl equations, the Helmholtz Equation for the electric field can be readily derived as:

$$
\nabla^{2} \vec{E}+k_{0}^{2} \varepsilon \vec{E}=0
$$

where $\mathrm{k}_{0}$ is the wavevector of the propagating wave in vacuum, defined as $\mathrm{k}_{0}=\omega / \mathrm{c}$. To separate the equation for evaluation, we must first define the geometry of the propagation. To begin, let us consider a "one-dimensional" problem where $\varepsilon$ varies only in one spatial-dimension and the wave propagates in the x-direction (Figure 9, below).

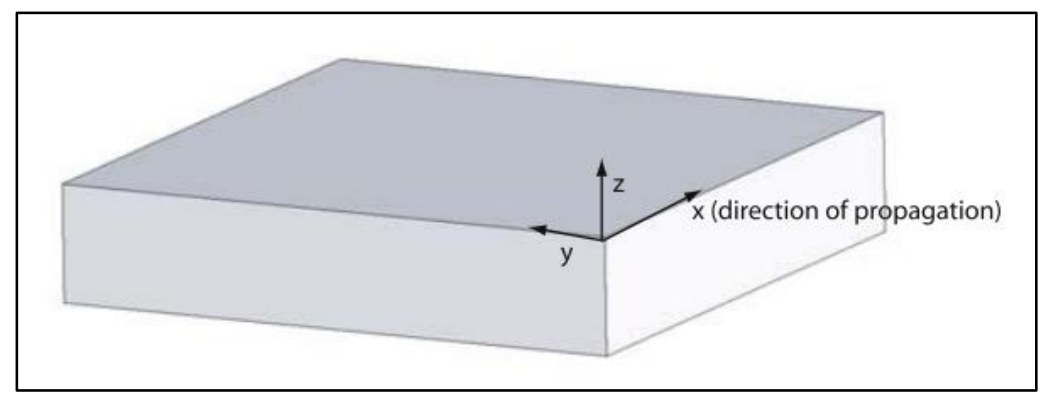

Figure 9: Illustration of the common planar waveguide geometry, with propagation in the x-direction.

- Reprinted from Plasmonics: Fundamental and Applications, by Stefan A. Maier, Springer Science + Business Media LLC, 2007 [15].

If the wave is also spatially uniform in the $y$-dimension, then $\vec{E}=\vec{E}(z)$. Setting the $z=0$ plane to the surface, $\overrightarrow{\mathrm{E}}$ for a propagating surface wave can be expressed as:

$$
\vec{E}(x, y, z)=\vec{E}(z) e^{i \beta x}
$$

where $\beta$ is the propagation constant. Inserting $\beta$ into the Helmholtz Equation for the electric field for our one-dimensional problem results in the familiar form of the wave equation for the electric field: 


$$
\frac{\partial^{2} \vec{E}(z)}{\partial z^{2}}+\left(k_{0}^{2} \varepsilon-\beta^{2}\right) \vec{E}=0
$$

A similar derivation would provide an associated wave equation for the magnetic field.

To calculate field profiles and dispersion relationships for the propagating waves, the above equation must be separated into respective differential equations for each component of the electric and magnetic fields. Using the Maxwell curl equations and the harmonic time dependence defined above (i. e. $\left.\frac{\partial}{\partial t}=-i \omega\right)$ the six field-component equations can be derived, simplified, and the system can be solved. As part of the simplification of the system of equations, recall that we already imposed that the propagation is only along the $x$-direction (i. e. $\left.\frac{\partial}{\partial x}=i \beta\right)$ and that the $y$-direction is constant (i.e. $\frac{\partial}{\partial y}=0$ ). In addition, recall we are interested in solutions which represent propagating waves confined to the metal-dielectric interface, which will only occur if it decays in the perpendicular z-direction [15].

From the simplified series of equations, there will arise two independent solutions, which will correspond to the transverse magnetic (TM) and transverse electric (TE) polarization modes for the propagating waves. In TM mode, there is no magnetic field in the plane of incidence, therefore $\mathrm{H}_{\mathrm{x}}=0$. Conversely, in TE mode, there is no electric field in the plane of incidence, thus $E_{x}=0$. The TM modes can be described by:

$$
\begin{aligned}
& E_{x}=-i \frac{1}{\omega \varepsilon_{0} \varepsilon} \frac{\partial H_{y}}{\partial z} \\
& \mathrm{E}_{\mathrm{z}}=-\frac{\beta}{\omega \varepsilon_{0} \varepsilon} \mathrm{H}_{\mathrm{y}}
\end{aligned}
$$


where $\varepsilon_{0}$ is the electric permittivity in vacuum and $\varepsilon$ is the relative permittivity of the material. Combining Equations 17.a and 17.b, the TM wave equation is expressed as:

$$
\frac{\partial^{2} \mathrm{H}_{\mathrm{y}}}{\partial^{2} \mathrm{z}}+\left(\mathrm{k}_{0}^{2} \varepsilon-\beta^{2}\right) \mathrm{H}_{\mathrm{y}}=0
$$

Conversely, the TE modes can be described by:

$$
\begin{aligned}
& H_{x}=i \frac{1}{\omega \mu_{0}} \frac{\partial E_{y}}{\partial z} \\
& \mathrm{H}_{\mathrm{z}}=\frac{\beta}{\omega \mu_{0}} \mathrm{E}_{\mathrm{y}}
\end{aligned}
$$

and therefore, the TE wave equation can be expressed as:

$$
\frac{\partial^{2} \mathrm{E}_{\mathrm{y}}}{\partial^{2} \mathrm{z}}+\left(\mathrm{k}_{0}^{2} \varepsilon-\beta^{2}\right) \mathrm{E}_{\mathrm{y}}=0
$$

These wave equations for the propagating surface modes can now be directly applied to the experimental setup to describe the surface plasmon polaritons, although for brevity the mathematical details will not be included. We should note that the gold layer in actual experiments is not a perfect-conductor and thus the SPPs will decay over time due to energy loses from damping of its free electrons and inter-band transitions [15].

\subsection{Excitation and Propagation}

For a surface plasmon polariton to be excited by a photon, the two must have the same frequency and momentum. Free-space photons have lower momentum than the SPPs which would be excited in the material with the same frequency. Thus, photons cannot directly excite SPPs. By coupling the photons into a prism or structures on the conducting surface (e.g. sharp edge, grating) the momentum match can be accomplished 
[13]. Here, diffraction at the edge of the gold layer — through the knife-edge effect—will be used to excite SPPs. As explained by the Huygens-Fresnel principle, in the knife-edge effect an object in the path of an electromagnetic wave will act as a secondary source and create a new wavefront which will propagate into the geometric shadow of the object [42]. It is important to note that the incident light waves will not only be spatially scattered but will also have an accompanying momentum change. A small fraction of the initial light will be diffracted such that its momentum will match that of SPPs at the given frequency which SPP excitation could occur. Thus, edge diffraction provides a method for SPP excitation in our gold layer, but it is a greatly inefficient process.

For SPP excitation in the vacuum-gold-ITO sample structure, a simplified approach — which is valid for thick metal layers $(\geq 100 \mathrm{~nm})$ - is to approximate the multi-layer system as two independent interfaces (Figure 10, below). In this case, the SPPs have two separate excitation modes: one at the top of the gold layer and a second at the bottom, which must be separately excited. Note: these modes will have significantly

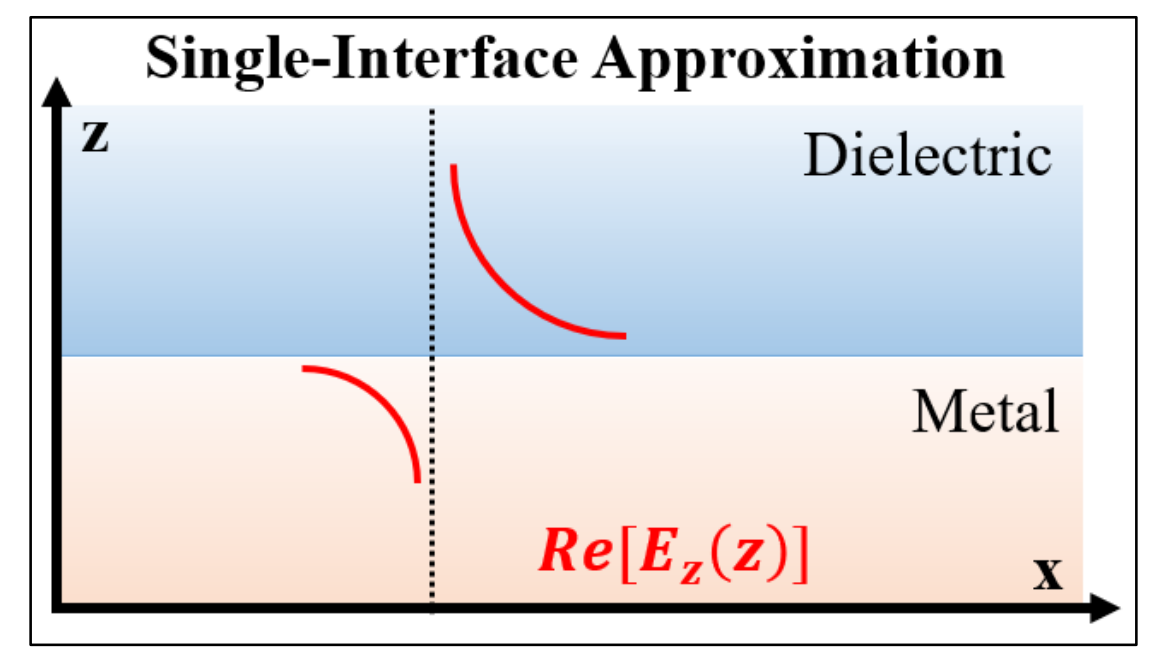

Figure 10: Illustration of the geometry and real portion of the electric field distribution for a single metal-dielectric interface. 
different speeds. For excitation with $\lambda=800 \mathrm{~nm}$ light, the vacuum-gold SPPs will travel near the speed of light $(0.98 \mathrm{c})$, with the ITO-gold SPPs traveling much slower $(0.53 \mathrm{c})$.

If we now consider the top interface, there are two half spaces to consider: (1) $\mathrm{z}>0$ which is comprised of a dielectric material with a positive and real dielectric constant $\left(\epsilon_{1}\right)$; and (2) $\mathrm{z}<0$ which is filled with a metallic material with a complex dielectric constant $\left(\epsilon_{2}\right)$. In addition, we require $\operatorname{Re}\left[\epsilon_{2}\right]<0$ since confined surface waves will only exist at interfaces between materials with dielectric constants with opposite signs. Applying equations 18.a, b, and c (above) for TE modes at the surface to the two adjoining regions and matching the boundary conditions results in a null-solution. Specifically, the tangential components of the electric field on either side of an interface between different materials must be equal and will thus cancel; as per Jackson's Classical Electrodynamics: $\mathbf{n} \times\left(\mathbf{E}_{2}-\mathbf{E}_{1}\right)=0$ (I.19) [11]. For TE mode polarization, all electric field components are tangential and thus no surface charge will be induced on the conductor. Thus, it is readily shown that no TE surface modes can exist and SPP excitation will only occur for partial- or pure-TM modes [15].

Conversely, the normal components of the displacement field on either side of an interface between different materials must be discontinuous; again, as per Jackson: $\left(\mathbf{D}_{2}-\mathbf{D}_{1}\right) \cdot \mathbf{n}=\sigma(\mathrm{I} .17)$ [11]. For partial- or pure-TM mode polarization, some or all electric field components are normal to the interface and thus a surface charge $(\sigma)$ will be induced on the conductor. By applying a similar technique as above to equations 17.a, b, and $\mathrm{c}$ (above) for TM modes at the surface, the propagation constant $(\beta)$ is shown to be: 


$$
\beta=k_{0} \sqrt{\frac{\epsilon_{1} \epsilon_{2}}{\epsilon_{1}+\epsilon_{2}}}
$$

where here $\epsilon_{1}$ is the dielectric constant of vacuum or ITO and $\epsilon_{2}$ is the complex dielectric constant of gold. Since $\epsilon_{2}$ is complex, so too is $\beta$. The wavevector (kspP) and the propagation length (LsPP) of the SPPs follow from $\beta$ :

$$
\begin{aligned}
& k_{S P P}=\operatorname{Re}[\beta] \\
& L_{S P P}=\frac{1}{2(\operatorname{Im}[\beta])}
\end{aligned}
$$

Values for kspP and LspP can be found in Table 3 (below).

While this approach is appealing for its simplicity, thick gold platelets aren't compatible with nano-scale structures or devices. A more robust approach—required for thin layers $(<100 \mathrm{~nm})$ — is to consider the entire multi-interface system

(Figure 11, below). In this case, the SPP excitation at the top of the layer and at its bottom need to be considered. When the metal layer is sufficiently thin $(<100 \mathrm{~nm})$ the

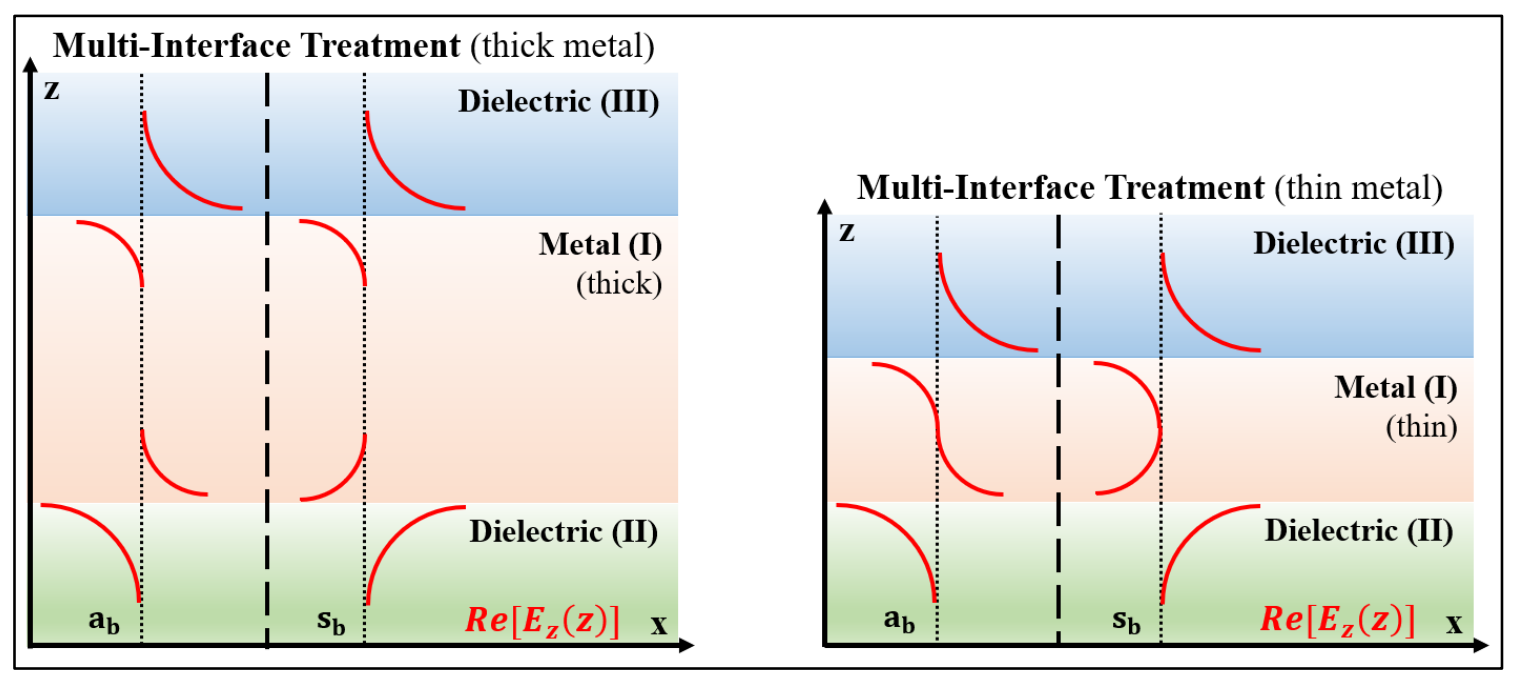

Figure 11: Illustration of the geometry and real portion of the electric field distribution for a multi-interface structure with a thick and thin metal layer. Note the two SPP modes are independent for the thick metal region but become hybridized for a thin metal region. 
two SPP modes will couple and a pair of hybridized symmetric $\left(\mathrm{s}_{\mathrm{b}}\right)$ and anti-symmetric $\left(\mathrm{a}_{\mathrm{b}}\right)$ modes will occur. For the sb mode, the electric field components in the conductor will cancel, resulting in the mode's electric field strength residing outside the conducting material. For the $a_{b}$ mode, the electric field components in the conductor will add together, resulting in the mode's electric field residing inside and outside the conducting material. Overall, these two modes have three significant differences: (1) momentum, (2) range, and (3) confinement [32]. These modes will again have significantly different speeds. For excitation with $\lambda=800 \mathrm{~nm}$ light, the $\mathrm{s}_{\mathrm{b}} \mathrm{SPP}$ mode will propagate near the speed of light $(0.98 \mathrm{c})$, with the $\mathrm{a}_{\mathrm{b}}$ SPP traveling far slower $(0.52 \mathrm{c})$. Not only is this approach more realistic, but it also corresponds to the thin gold platelets which are readily synthesized in the lab with thicknesses $\sim 50 \mathrm{~nm}[16]$.

To calculate the hybridized symmetric $\left(\mathrm{s}_{\mathrm{b}}\right)$ and anti-symmetric $\left(\mathrm{a}_{\mathrm{b}}\right)$ modes, we must graphically solve the dispersion relationship, which links the propagation constant $(\beta)$ and the frequency ( $\left.\omega_{\text {SPP }}\right)$ of the SPPs. From literature [15], the dispersion relation can be expressed as:

$$
e^{-4 k_{1} a}=\frac{\left(k_{1} / \epsilon_{1}\right)+\left(k_{2} / \epsilon_{2}\right)\left(k_{1} / \epsilon_{1}\right)+\left(k_{3} / \epsilon_{3}\right)}{\left(k_{1} / \epsilon_{1}\right)-\left(k_{2} / \epsilon_{2}\right)\left(k_{1} / \epsilon_{1}\right)-\left(k_{3} / \epsilon_{3}\right)}
$$

where $\mathrm{k}_{\mathrm{i}}$ is the wavevector and $\epsilon_{\mathrm{i}}$ is the relative permittivity for the associated material region $(i=1,2,3)$. As $\epsilon_{i}$ may include both real and imaginary components, we can separate Equation 22 into two separate solvable equations:

$$
R e\left[e^{-4 k_{1} a}-\frac{\left(k_{1} / \epsilon_{1}\right)+\left(k_{2} / \epsilon_{2}\right)\left(k_{1} / \epsilon_{1}\right)+\left(k_{3} / \epsilon_{3}\right)}{\left(k_{1} / \epsilon_{1}\right)-\left(k_{2} / \epsilon_{2}\right)\left(k_{1} / \epsilon_{1}\right)-\left(k_{3} / \epsilon_{3}\right)}\right]=0
$$




$$
\operatorname{Im}\left[\mathrm{e}^{-4 \mathrm{k}_{1} \mathrm{a}}-\frac{\left(\mathrm{k}_{1} / \epsilon_{1}\right)+\left(\mathrm{k}_{2} / \epsilon_{2}\right)\left(\mathrm{k}_{1} / \epsilon_{1}\right)+\left(\mathrm{k}_{3} / \epsilon_{3}\right)}{\left(\mathrm{k}_{1} / \epsilon_{1}\right)-\left(\mathrm{k}_{2} / \epsilon_{2}\right)\left(\mathrm{k}_{1} / \epsilon_{1}\right)-\left(\mathrm{k}_{3} / \epsilon_{3}\right)}\right]=0
$$

Each of the above equations will have two solutions, for a total of four curves

(Figure 12, below). If we graph Equations 23.a and b, their intersections will represent SPP modes: both the $\mathrm{s}_{\mathrm{b}}$ and $\mathrm{ab}$ modes if they exist. If we plot the equations with the proper dimensions, the intersection points will produce the propagation length $(\mathrm{L})$ and the real portion of the effective index of refraction of the conductor $\left(\mathrm{n}_{\text {eff }}\right)$. As an example

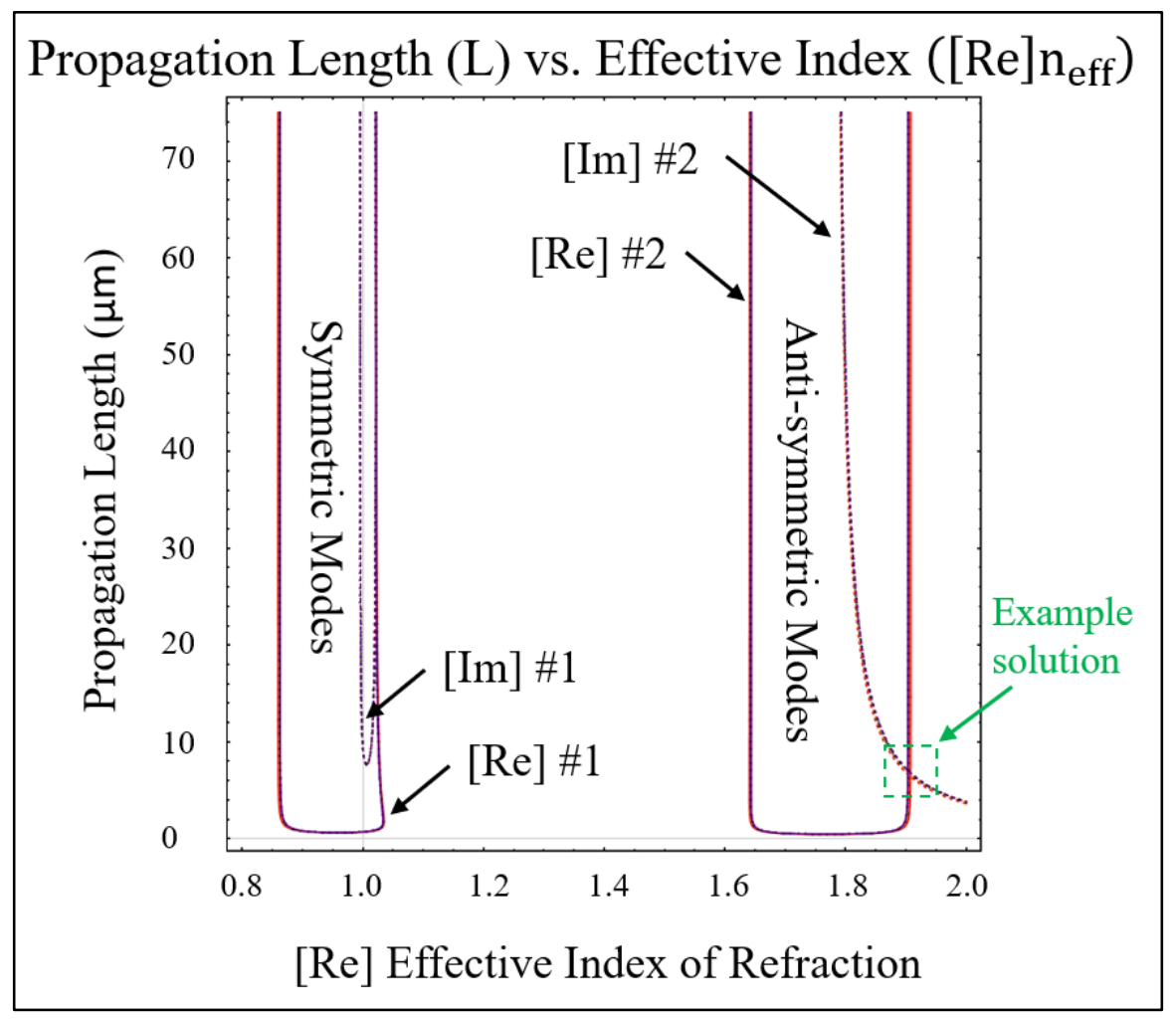

Figure 12: Graphical solutions for the symmetric $\left(\mathrm{s}_{\mathrm{b}}\right)$ and anti-symmetric $\left(\mathrm{a}_{\mathrm{b}}\right)$ SPP modes for $\lambda=800 \mathrm{~nm}$ light incident onto a vacuum-gold-ITO multi-layer interface for gold of thickness $T=\{60,70,80,90,100 \mathrm{~nm}\}$. The solid lines represent solutions to the real portion of the dispersion relationship and the dotted lines the imaginary portions. The plots were calculated via Mathematica. 
Figure 13 (below) shows the graphical solution for the $a_{b}$ modes for $\lambda=800 \mathrm{~nm}$ light incident on a vacuum-gold-ITO sample with gold layer thicknesses between $\mathrm{T}=\{60: 100 \mathrm{~nm}\}$. The solid lines represent solutions to the real portions of the dispersion relationship and the dotted lines the imaginary portions. The graphical solutions were calculated using Wolfram Mathematica programs adapted with permission from Dr. Robert Word.

Using this technique, we can determine the relationship between the thickness of the gold layer and the propagation length of the excited SPPs for both the symmetric $\left(\mathrm{s}_{\mathrm{b}}\right)$ and anti-symmetric $\left(\mathrm{a}_{\mathrm{b}}\right)$ modes. As we can see in Figure 14 (below), the sb SPP mode will

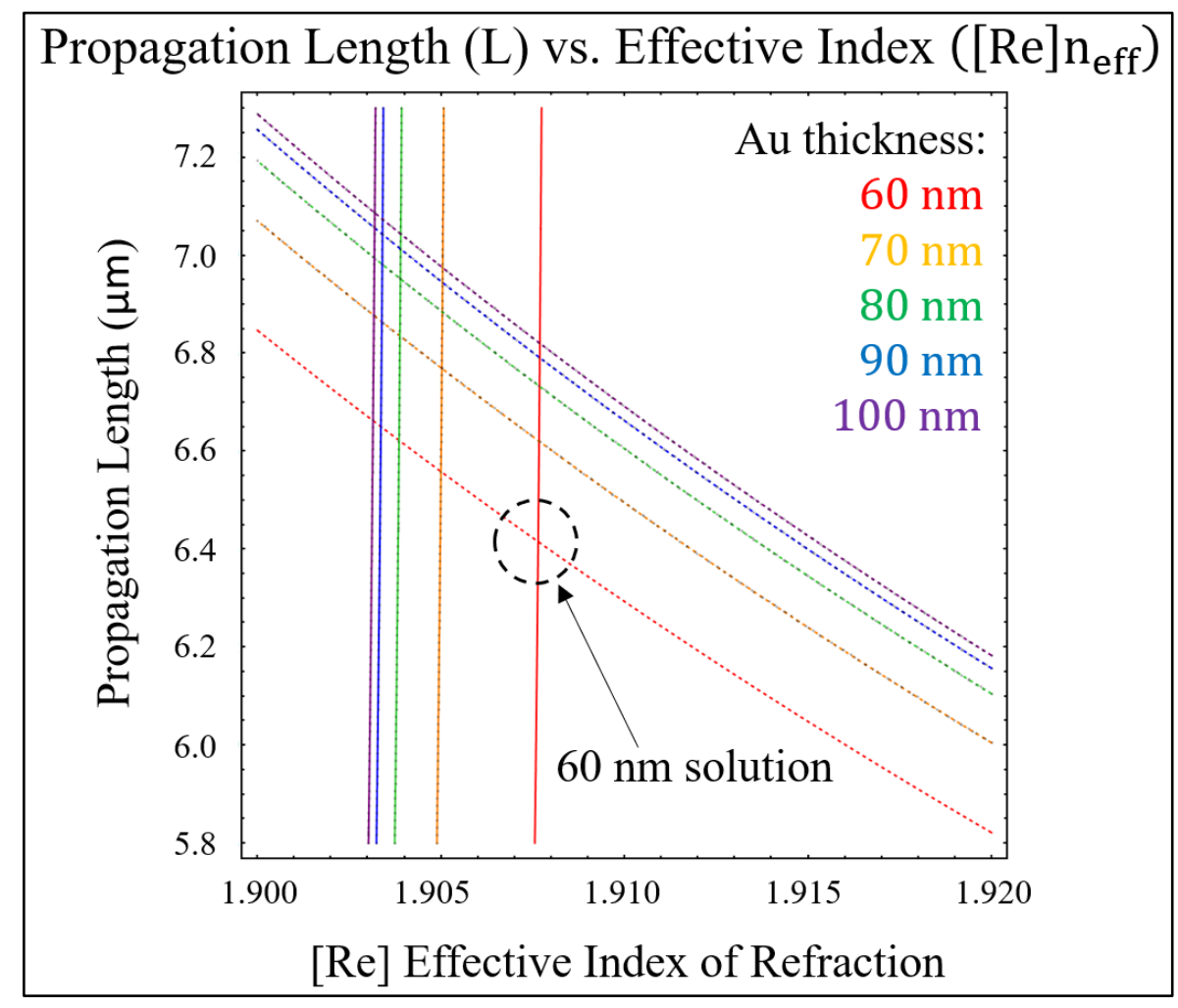

Figure 13: Graphical solutions for the anti-symmetric SPP modes for $\lambda=800 \mathrm{~nm}$ light incident onto a vacuum-gold-ITO multi-layer interface for gold of thickness $\mathrm{T}=\{60,70,80,90,100 \mathrm{~nm}\}$. The solid lines represent solutions to the real portions of the dispersion relationship and the dotted lines the imaginary portions. 
cutoff at $<50 \mathrm{~nm}$ thickness $(\sim 48 \mathrm{~nm})$, while the ab mode exists for thinner gold layers.

A summary of the propagation characteristics of the non-hybridized (top / bottom) and hybridized $\left(\mathrm{s}_{\mathrm{b}} / \mathrm{a}_{\mathrm{b}}\right)$ SPP modes and the expected interference spacing for $\lambda=800 \mathrm{~nm}$ light incident at $\theta=60^{\circ}$ to the surface normal is provided below in Table 3 . As can be seen below, for $\lambda=800 \mathrm{~nm}$ light and our sample combination of vacuum / singlecrystalline gold / ITO the more basic treatment of single-interface calculations is an excellent approximation. Our results from calculating the multi-interface hybridized modes show that the speeds, wavelengths, and interference spacings for the $\mathrm{s}_{\mathrm{b}}$ and $\mathrm{ab}$ SPP

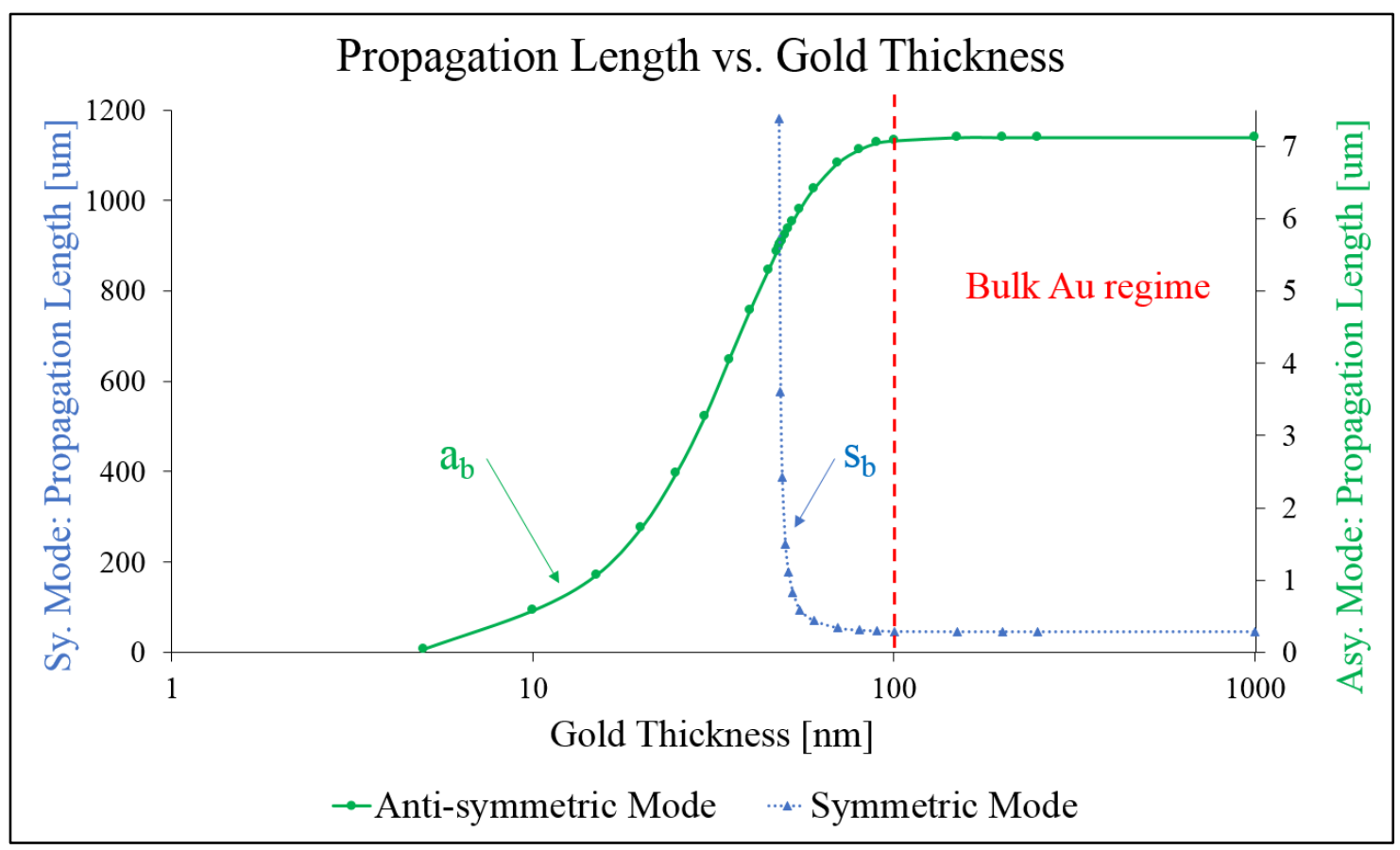

Figure 14: Graph of the propagation length vs. gold thickness for the anti-symmetric (green, solid) and the symmetric (blue, dotted) SPP modes excited by $\lambda=800 \mathrm{~nm}$ light on a planar vacuum-gold-ITO sample. Above $\sim 100 \mathrm{~nm}$ the two hybridized SPP modes remain constant with increasing gold thickness. 
modes are nearly identical for both thick and thin gold layers; with each of the listed characteristics of the comparable modes within $1 \%$ of each other. If further calculations required us to use the propagation length (L) of the SPP modes, then the advanced treatment would be required.

\begin{tabular}{|l|c|c|c|c|}
\hline Mode & $\begin{array}{c}\text { Speed } \\
{[\% \mathrm{c}]}\end{array}$ & $\begin{array}{c}\lambda \\
{[\mathrm{nm}]}\end{array}$ & $\begin{array}{c}\text { Propagation } \\
\text { Length }[\mu \mathrm{m}]\end{array}$ & $\begin{array}{c}\text { Interference } \\
\text { Spacing }[\mu \mathrm{m}]\end{array}$ \\
\hline Non-hybridized: vacuum / gold & 0.979 & 783.1 & 46.1 & 5.15 \\
\hline Non-hybridized: ITO / gold & 0.525 & 420.2 & 7.13 & 0.77 \\
\hline Hybridized: symmetric $(\mathrm{sb})$ & 0.980 & 784.3 & 239.9 & 5.20 \\
\hline Hybridized: anti-symmetric $\left(\mathrm{ab}_{\mathrm{b}}\right)$ & 0.524 & 418.9 & 5.77 & 0.77 \\
\hline
\end{tabular}

Table 3: Calculated speed (s), wavelength ( $\lambda$ ), and propagation length (L) for SPP modes of non-hybridized single-interfaces of vacuum-gold and gold-ITO and hybridized multi-layer vacuum-gold-ITO interfaces with $\lambda=800 \mathrm{~nm}$ incident light. The interference spacing values are for $\lambda=800 \mathrm{~nm}$ light obliquely incidence at $\theta=60^{\circ}$.

It is important to note that the graphical calculations for solving the dispersion relationship for the multi-layer vacuum-gold-ITO sample do not consider the thickness of the ITO dielectric substrate. In addition, the imaginary portion of the index of refraction of ITO was not included. The ITO samples have a thickness of $\sim 275 \mathrm{~nm}$, which is thicker than the expected penetration depth for the anti-symmetric (ab) SPP mode. Still, it is possible that the ITO layer could act as a waveguide for light coupled in from diffraction at the edge of the gold region (or from other surface defects), which would make its effective index of refraction lower than the assumed value of $1.77\left(1.5<\mathrm{n}_{\mathrm{eff}}<1.77\right)$. Additional details of the derivation and calculation of hybridized SPP modes are available in literature [15], [32]. 


\section{Results and Discussion}

\subsection{Experimental Results}

PEEM micrographs previously taken in our lab were used as experimental guidelines and motivations for the computer simulations. The micrographs were obtained from a random deposition of gold microstructures, which included rods, triangles, and hexagons (Figure 15, below). These included both equilateral and truncated polygons.

PEEM micrographs were obtained for both transverse electric (TE) and transverse magnetic (TM) polarizations for obliquely incident light and for both $\varphi=0^{\circ}$ and $\varphi=90^{\circ}$ polarizations for normally incident light. Let us begin with oblique incidence $\left(\theta=60^{\circ}\right)$. In Figure 16 (a / c, below), an array of gold structures is illuminated with (a) TM and (c) TE polarized, $\lambda=800 \mathrm{~nm}$ light. If we digitally magnify the TM polarized imaged (a) to view an individual triangular platelet within the distribution, localized photoemission

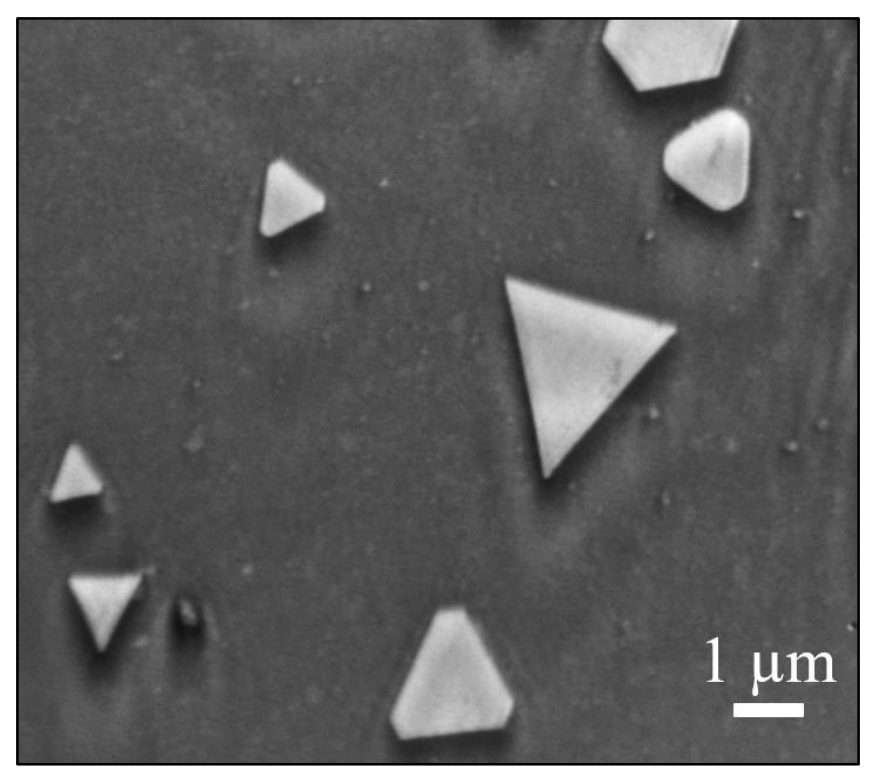

Figure 15: PEEM micrograph of an array of gold microstructures excited with $\lambda=244 \mathrm{~nm} \mathrm{CW}$ light incident at $\theta=60^{\circ}$. Micrograph provided by Dr. Robert C. Word. 
is visible from discrete regions within the gold material; specifically, the rear tip and to a lesser extent the exterior edges (Figure 16.b; below). Note: the asymmetry of the tip emission is due to a $19^{\circ}$ offset between the plane of incidence and the long axis of the platelet. TE polarization shows almost no photoemission here (Figure 16.c, d; below). Figure 16 (below) is presented as the $4^{\text {th }}$-root of the photoemission yield, which will correspond to the $|\vec{E}|^{2}$ for 4-photon processes.

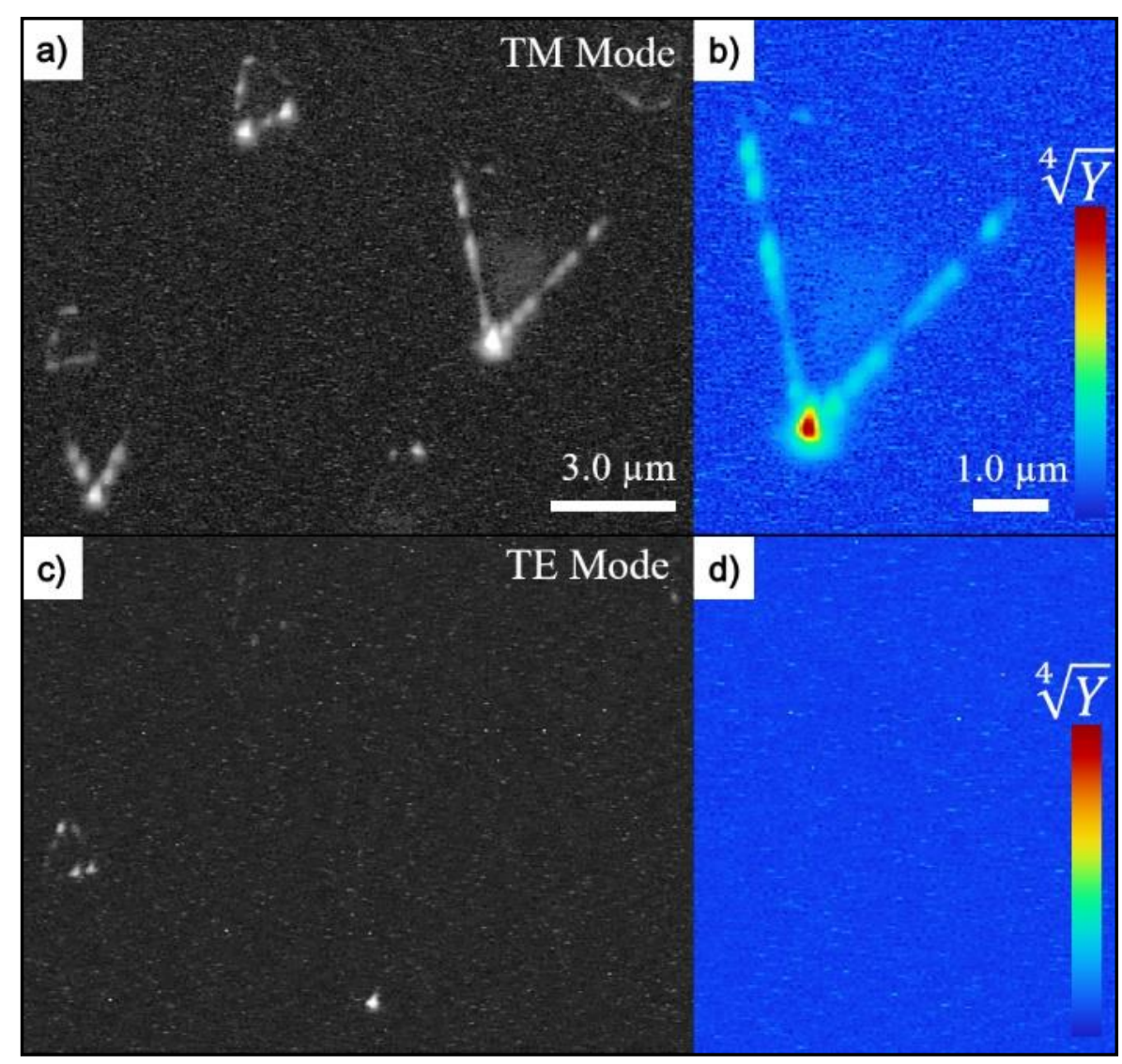

Figure 16: PEEM micrographs of gold microstructures excited by $\lambda=800 \mathrm{~nm}$ light incident at $\theta=60^{\circ}$ for TM-(a) original, (b) digitally magnified and colored —and TE polarizations $\{(\mathrm{c}),(\mathrm{d})\}$. The magnified triangular platelet is equilateral with a length of $\sim 2.9 \mu \mathrm{m}$. Contrast ranges for the micrographs: $(\mathrm{a} / \mathrm{b}) \equiv \mathrm{C}_{\mathrm{ab}}=\{1256: 40875\}$, $(c)=0.9 \cdot C_{a b}$, and $(d)=0.5 \cdot C_{a b}$. Note: TE excitation shows no photoemission here. 
Next, let us consider normal incidence $\left(\theta=0^{\circ}\right)$ illumination. In Figure 17 (below), a single triangular gold platelet is illuminated with (a) $\varphi=0^{\circ}$ and (b) $\varphi=90^{\circ}$ polarizations, both with $\lambda=800 \mathrm{~nm}$ light. A semi-uniform photoemission pattern is seen along the edges for both polarizations, with minimal emission otherwise. The polarization dependent moiré pattern visible in PEEM was previously explained by our group [26].

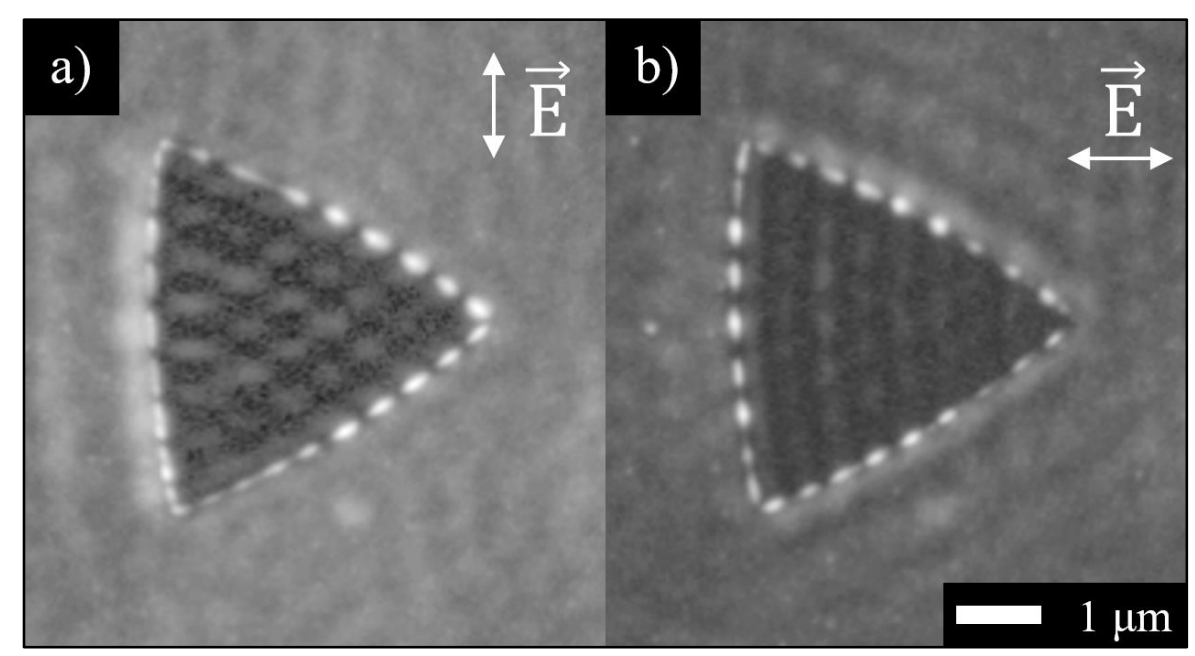

Figure 17: Normal incidence $\left(\theta=0^{\circ}\right)$ PEEM micrographs of triangular gold platelets for $\lambda=800 \mathrm{~nm}$ light with (a) $\varphi=0^{\circ}$ and (b) $\varphi=90^{\circ}$ polarizations. Note: The micrographs are presented as the $3^{\text {rd }}$ root of the photoemission yield.

- Adapted with permission from Photonic and plasmonic surface field distributions characterized with normal- and oblique-incidence multi-photon PEEM, by R.C. Word and R. Könenkamp, Ultramicroscopy, vol. 183, pp. 1139-1351, 2017 [26]. 


\subsection{2-Dimensional Simulation Results}

Using the RF-Module of COMSOL Multiphysics (version 4.3), computer simulations were performed for a variety of gold microstructures. Most of the simulation results are for 3-dimensional structures, as will be discussed in Section 5.3, but first we present simplified 2-dimensional results which allow us to characterize centrally propagating surface modes. We are considering here a 2 -dimensional vertical cross section of the sample with infinite extension in the direction perpendicular to the crosssection (y-axis) for a gold region with length $\mathrm{L}$ and thickness $\mathrm{T}$ within the cross-sectional plane (Figure 18, below). At the top surface of the gold region we expect both standing and traveling SPP modes to exist.

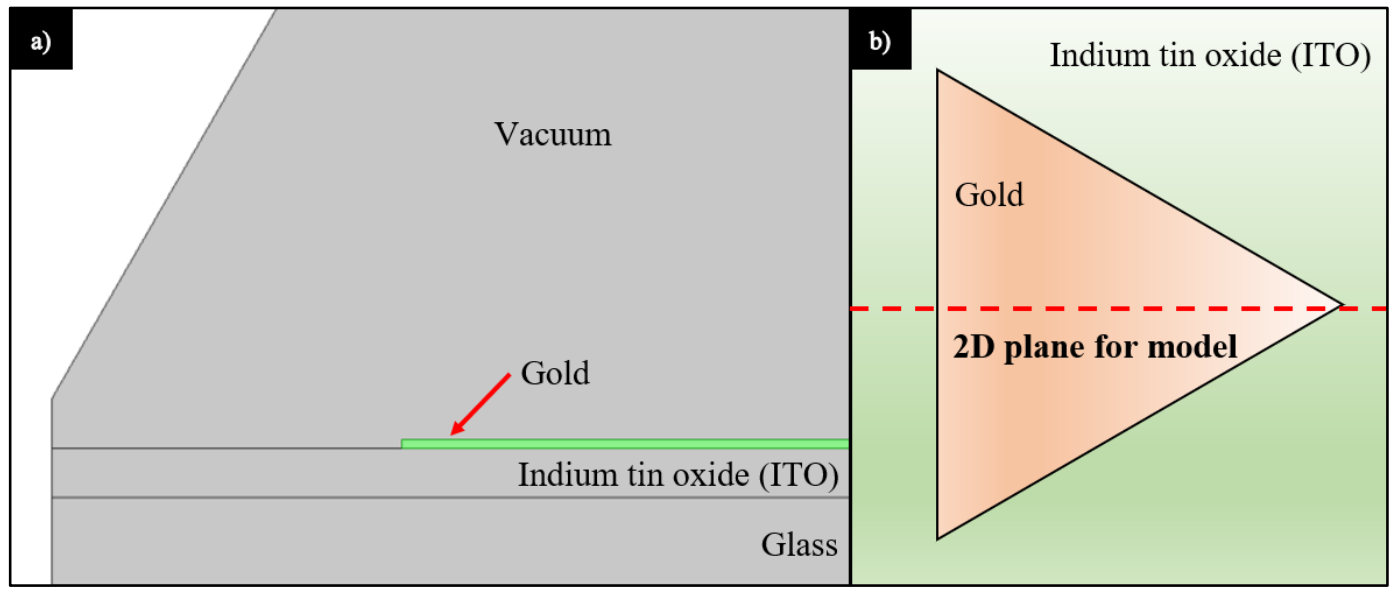

Figure 18: (a) Cross-sectional view of a 2D COMSOL model of a vacuum-gold-ITOglass sample with the layers labeled and the gold region highlighted in green and (b) a top down illustration of the triangular gold structure with the 2D plane of simulation marked with a dashed line (red). 


\subsubsection{Interference Spacing Derivation}

To begin, let us consider the expected traveling mode for the triangular gold platelets characterized in PEEM. As discussed in Section 2.1, a time-averaged PEEM micrograph will display time-independent interference patterns. For the single metal-dielectric interface approximation—valid for thick conducting layers $(\geq 100 \mathrm{~nm})$ we know from Section 4.2 that the surface plasmon polaritons excited with $\lambda=800 \mathrm{~nm}$ light and traveling along the vacuum-gold interface will have a speed of $0.98 \mathrm{c}$. From this information, we are able to calculate the interference spacing between SPPs excited at the edge of the gold platelet and the incident light from the laser as derived below

(Figure 19, below). In this case the light is incident to the sample at an angle $\theta$ (relative to

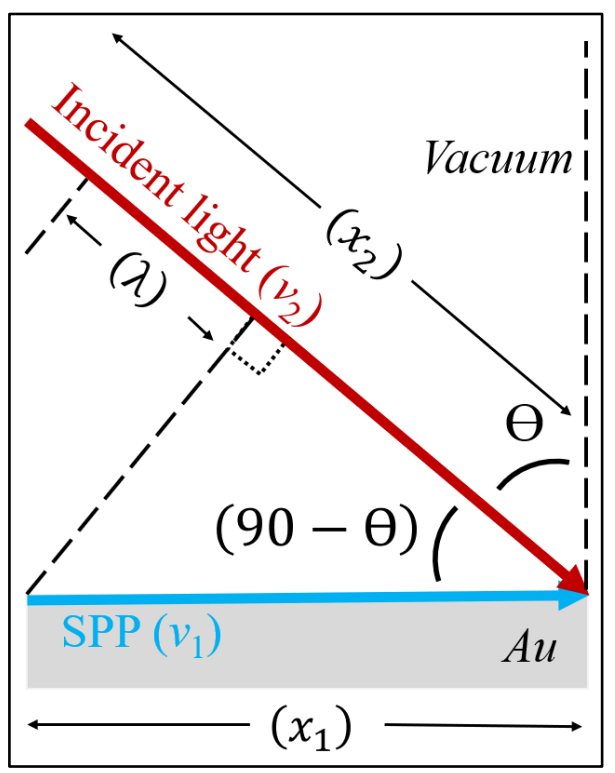

Figure 19: Illustration of the experiment geometry of a vacuum-gold SPP (blue) traveling along the gold surface to interfere with light of wavelength $\lambda$ (red) incident at an angle relative to the surface normal of $\theta . \mathrm{x}_{1}$ is the distance traveled by the SPP between the edge of the gold region and the location of the next interference maximum with the incident light and $\mathrm{x}_{2}$ is the angled distance traveled by the incident light between the edge of the gold region and the sample surface. 
the surface normal) and the excited SPPs will travel across the surface of the gold. The distances $\mathrm{x}_{1}$ and $\mathrm{x}_{2}$ will represent the distance traveled by the SPPs and the distance traveled by the incident light as measured between the edge of the gold region and the location of the next interference maximum.

First, let us determine expressions for the distances traveled by the SPPs $\left(\mathrm{x}_{1}\right)$ and the incident light $\left(\mathrm{x}_{2}\right)$ for the time between excitation and interference $(\mathrm{t})$ :

$$
\mathrm{t}=\frac{\mathrm{x}_{1}}{\mathrm{v}_{1}} \quad \mathrm{t}=\frac{\mathrm{x}_{2}}{\mathrm{v}_{2}}=\frac{\lambda+\mathrm{x}_{1} \cos (90-\theta)}{\mathrm{v}_{2}}
$$

Next, we can combine the two equations and solve for $\mathrm{x}_{1}$ :

$$
\frac{x_{1}}{v_{1}}=\frac{\lambda+x_{1} \cos (90-\theta)}{v_{2}} \quad x_{1}=\frac{\lambda\left(v_{1}\right)}{v_{2}-v_{1} \cos (90-\theta)}
$$

Substituting in $\lambda=800 \mathrm{~nm}, \mathrm{v}_{1}=0.98 \mathrm{c}, \mathrm{v}_{2}=\mathrm{c}$, and $\theta=60^{\circ}$, we can calculate the interference spacing:

$$
\begin{gathered}
\mathrm{x}_{1}=\frac{\left(800 \times 10^{-9}[\mathrm{~m}]\right) \cdot\left(2.94 \times 10^{8}[\mathrm{~m} / \mathrm{s}]\right)}{\left(3.0 \times 10^{8}[\mathrm{~m} / \mathrm{s}]\right)-\left(2.94 \times 10^{8}[\mathrm{~m} / \mathrm{s}]\right) \cdot \cos (90-60)} \\
\mathrm{x}_{1}=5.15 \mu \mathrm{m}\left(\mathrm{s}_{\mathrm{b}} \mathrm{SPP} \text { mode for thick } \mathrm{Au}\right) \\
\text { or }
\end{gathered}
$$

\section{$5.20 \mu \mathrm{m}\left(\mathrm{s}_{\mathrm{b}}\right.$ SPP mode for thin $\left.\mathrm{Au}\right)$}

A simple, alternative method for calculating the interference spacing $\left(\mathrm{x}_{\mathrm{i}}\right)$ is to calculate the difference between the wavevector of the SPP mode $\left(\mathrm{k}_{\mathrm{spp}}\right)$ and the in-plane wavevector of the incident source light $\left(\mathrm{k}_{\mathrm{s}}\right)$. We can equate these terms as: 


$$
\begin{gathered}
k_{i}=k_{s p p}-k_{s}=\frac{2 \pi}{\lambda_{s p p}}-\frac{2 \pi}{\lambda_{s}} \\
k_{i}=\frac{2 \pi}{0.784[\mu m]}-\frac{2 \pi}{\left(0.8[\mu m] / \cos \left(30^{\circ}\right)\right)} \\
k_{i}=1.21[1 / \mu m] \\
\rightarrow \mathrm{x}_{\mathrm{i}}=2 \pi / \mathrm{k}_{\mathrm{i}}=5.18 \mu \mathrm{m}(\mathrm{sb} \text { SPP mode for thin } \mathrm{Au})
\end{gathered}
$$

If we assume a perfect conductor with zero resistance or loss, this interference pattern would continue over the entire gold layer. As the gold in the experiment will not be a perfect conductor, the SPPs will gradually lose energy, thus steadily reducing in amplitude, and the interference pattern will lose contrast at longer distances. As the incident light beam and the excited SPPs have the same frequency, their interference pattern will not propagate. In other words, there will be locations of constructive interference where the amplitude will periodically vary and there will be stationary nodes between them of constant amplitude. As the images in PEEM are time-averaged, we will not see any propagating modes, but the standing interference pattern (generated during the laser pulse duration) will be clearly visible. 


\subsubsection{Unbounded Surfaces + FFT Analysis}

Now, to confirm the interference spacing for the single-interface approximation or identify the more complicated surface modes for the multi-interface geometry, we can create a simple 2-dimensional model in COMSOL (Figure 20, below). In the zoomed image of the simulated electric field intensity in Figure 20.b (below), the $\sim 5.2 \mu \mathrm{m}$ interference spacing previously derived is clearly visible along the gold surface.

This model can be used for both thicknesses of gold by simply adjusting the gold layer between a thick, bulk layer $(\mathrm{T}=1 \mu \mathrm{m})$ or a thin, more realistic layer $(\mathrm{T}=50 \mathrm{~nm})$. Note: A 2D model will show the stationary interference pattern at the sample surface generated by the incoming light and the traveling surface plasmon. As the $2 \mathrm{D}$ plane is normal to the sample plane, the model will not account for any edge effects, but due to memory constraints the model must be $2 \mathrm{D}$ to consider large platelet lengths $(>\sim 10 \mu \mathrm{m})$.

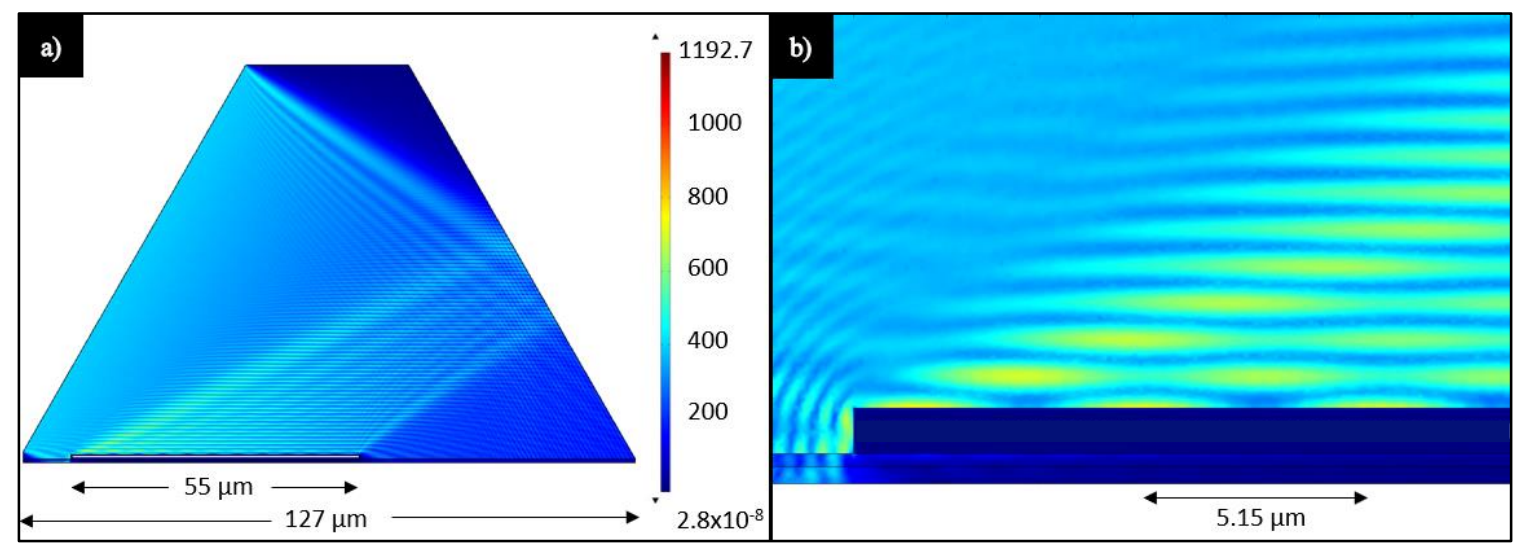

Figure 20: 2D simulation results of the time-averaged electric field for $\lambda=800 \mathrm{~nm}$ light obliquely incident at $\theta=60^{\circ}$ to the surface normal with TM polarization for a thick gold layer $(\mathrm{T}=1 \mu \mathrm{m})$ on top of a planar ITO-glass stack (surrounded by a vacuum region): (a) simulation results for the full model and (b) a zoomed image of the surface of the gold layer. The $\sim 5.2 \mu \mathrm{m}$ interference spacing between a SPP mode excited at the edge of the gold layer and the incident light calculated above is visible along the gold surface. 
To extrapolate mode information from the model, we can export the time-averaged electric field across the surface of the gold or ITO layers and perform a spatial fast Fourier transform (FFT). The FFT calculations were performed using Wolfram Mathematica programs adapted with permission from Theodore Stenmark (Figure 21, below). For FFT analysis, the length of the platelet should be many times larger than the distance of any identified modes, so we will begin by analyzing a platelet with $\mathrm{L}=50 \mu \mathrm{m}$ (i.e. $\sim 10 \mathrm{X}$ the expected spacing of $\sim 5.2 \mu \mathrm{m}$ ).

Let us begin with the gold surface. As we can see in the FFT periodogram (Figure 21.b; below), we have a dominant periodicity at $5.14 \pm 0.46 \mu \mathrm{m}$ for both $50 \mathrm{~nm}$ and $1 \mu \mathrm{m}$ gold layers, which we correlate to a surface interference between the SPPs excited at the gold edge and the light incident at the gold surface (theory: $5.2 \mu \mathrm{m}, 0.8 \%$ error).

In addition, for both thicknesses there is a second, much weaker periodicity at $423.7 \pm 3.6 \mathrm{~nm}$ which we correlate to interference between SPPs reflected back towards the front of the gold layer from the rear edge and the oblique incident light (theory: $423.9 \mathrm{~nm}, 0.05 \%$ error). The amplitude of this peak was significantly decreased in the FFT plot by implementing a curved rear edge, which reduced diffraction backward from the rear edge of the gold material. Both these interference periodicities are visible in the electric field vs. distance plot (Figure 21.a, below) with the $\sim 5.2 \mu \mathrm{m}$ spacing forming the dominant oscillation pattern and the $\sim 420 \mathrm{~nm}$ spacing forming fine oscillations (or "ripples") within the overall oscillation. The broad, weaker peak at $\sim 2.6 \mu \mathrm{m}$ is an artifact of the FFT analysis. The decomposition of the $\sin ^{2}$ function introduces a false peak at 


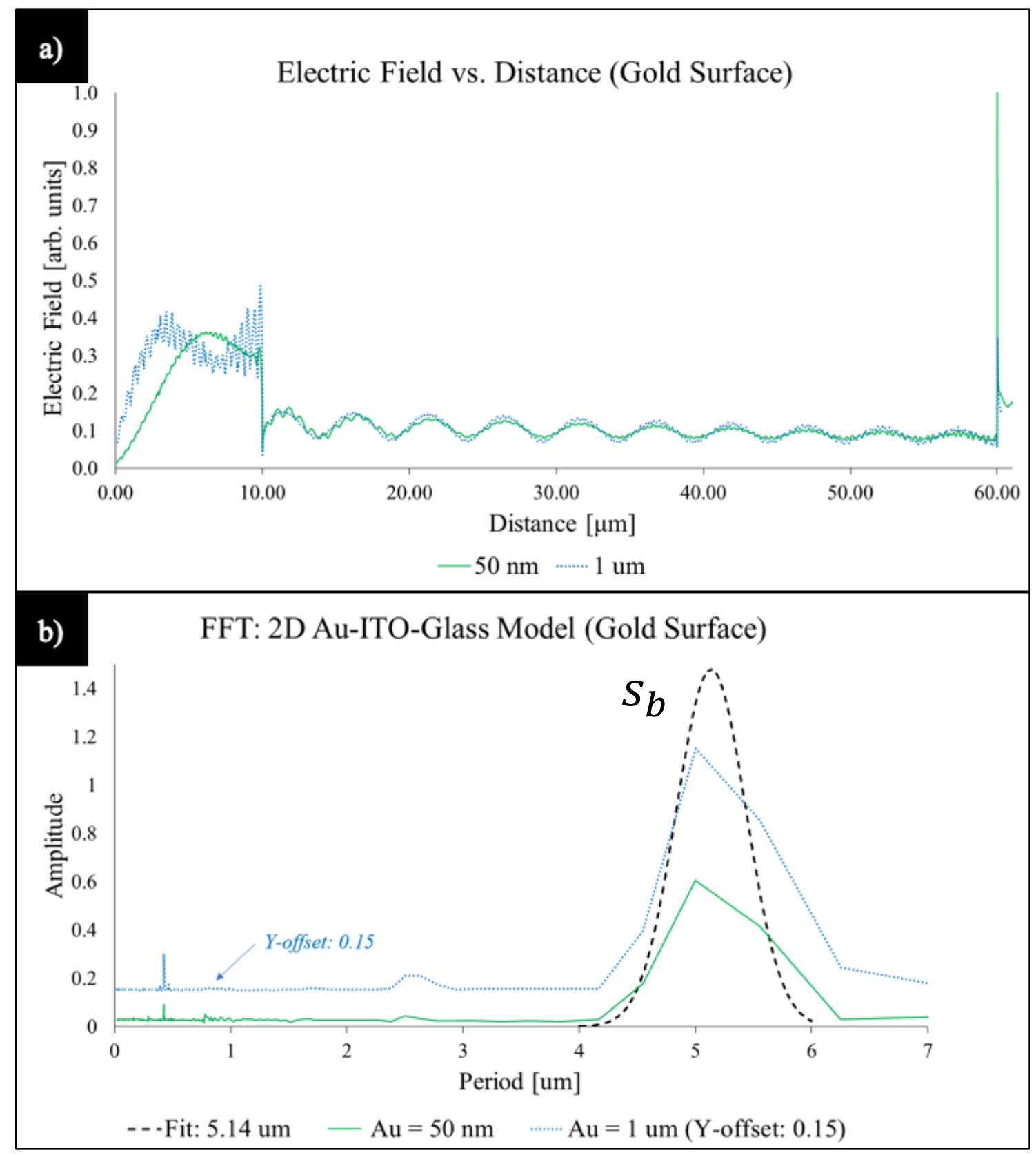

Figure 21: (a) Graph of the time-averaged electric field squared at the gold surface vs. distance from the boundary of the model for a $50 \mu \mathrm{m}$ long gold platelet exposed to $\lambda=800 \mathrm{~nm}$ light with oblique incidence $\left(\theta=60^{\circ}\right)$ and TM polarization for thin (50 nm; green, solid) and thick ( $1 \mu \mathrm{m}$; blue, dotted) gold layers; (b) FFT analysis of the electric field data plotted in (a). A single periodicity is visible for both $50 \mathrm{~nm}$ and $1 \mu \mathrm{m}$ thick gold layers at $\sim 5.2 \mu \mathrm{m}$, which is attributed to the symmetric SPP mode interfering with the incident light. FFT data calculated via Mathematica. The normal distribution (black, dashed) was fit to the data sets before a $y_{\text {shift }}=0.15$ was applied to the $1 \mu \mathrm{m}$ curve. 
exactly half the period of the main peak at $\sim 5.2 \mu \mathrm{m}$. FFT analysis was completed on gold regions ranging from $20-60 \mu \mathrm{m}$ in length. The two modes at $\sim 420 \mathrm{~nm}$ and $\sim 5.2 \mu \mathrm{m}$ were clearly visible for all lengths. At $10 \mu \mathrm{m}$ length, the $\sim 420 \mathrm{~nm}$ mode is still visible in FFT, but the $\sim 5.2 \mu \mathrm{m}$ mode can't be resolved as the region's length is comparatively too short.

Next, we will look at the ITO surface (i.e. bottom of the gold layer). As we can see in the FFT periodogram (Figure 22, below), a thick $1 \mu \mathrm{m}$ gold layer shows one dominant periodicity, while a thin $50 \mathrm{~nm}$ gold layer shows two distinct periodicities. For the thick $1 \mu \mathrm{m}$ gold, no symmetric SPP mode $(\mathrm{sb})$ interference is seen at the ITO surface, as expected due to the confinement of the sb mode at the gold surface. Interference from the anti-symmetric SPP mode $\left(a_{b}\right)$ is seen at the ITO surface with a periodicity of $1.72 \pm 0.06 \mu \mathrm{m}$, which is attributed to the $\mathrm{a}_{\mathrm{b}}$ mode interfering with light propagating in the ITO layer (theory: $1.72 \mu \mathrm{m}$ ). For light propagating in the ITO layer beneath the gold, the effective index of the material will be between that of the glass substrate $(n=1.45)$ and the ITO material in vacuum $(n=1.77)$. Prior work from the group indicates a $\lambda=\sim 540 \mathrm{~nm}$ for a guided mode in the ITO layer, which would correspond to an effective index of $\mathrm{n}_{\mathrm{eff}}=1.48$. This would produce an interference periodicity of $1.86 \mu \mathrm{m}$, which is close to our experimental result (7.5\% error).

For the thin $50 \mathrm{~nm}$ gold, symmetric SPP mode $\left(\mathrm{s}_{\mathrm{b}}\right)$ interference is still seen at the ITO surface albeit shifted to a periodicity of $5.56 \pm 0.63 \mu \mathrm{m}$, which is attributed to SPP penetration through the thin gold film (theory: $5.2 \mu \mathrm{m}, 7.3 \%$ error). This slightly higher periodicity falls well within the uncertainty of the experimental results. Interference from the anti-symmetric SPP mode $(a b)$ is seen at the ITO surface with a periodicity of 
$862 \pm 14.9 \mathrm{~nm}$, which is attributed to interference between the $\mathrm{ab}_{\mathrm{b}}$ and sb SPP modes

(theory: $901 \mathrm{~nm}, 4.4 \%$ error) in the thin gold layer. This periodicity also falls well within the uncertainty of the experimental results.

Lastly, the minor peaks are attributed to optical diffraction and reflection patterns from light in the ITO and the vacuum for both gold thicknesses.

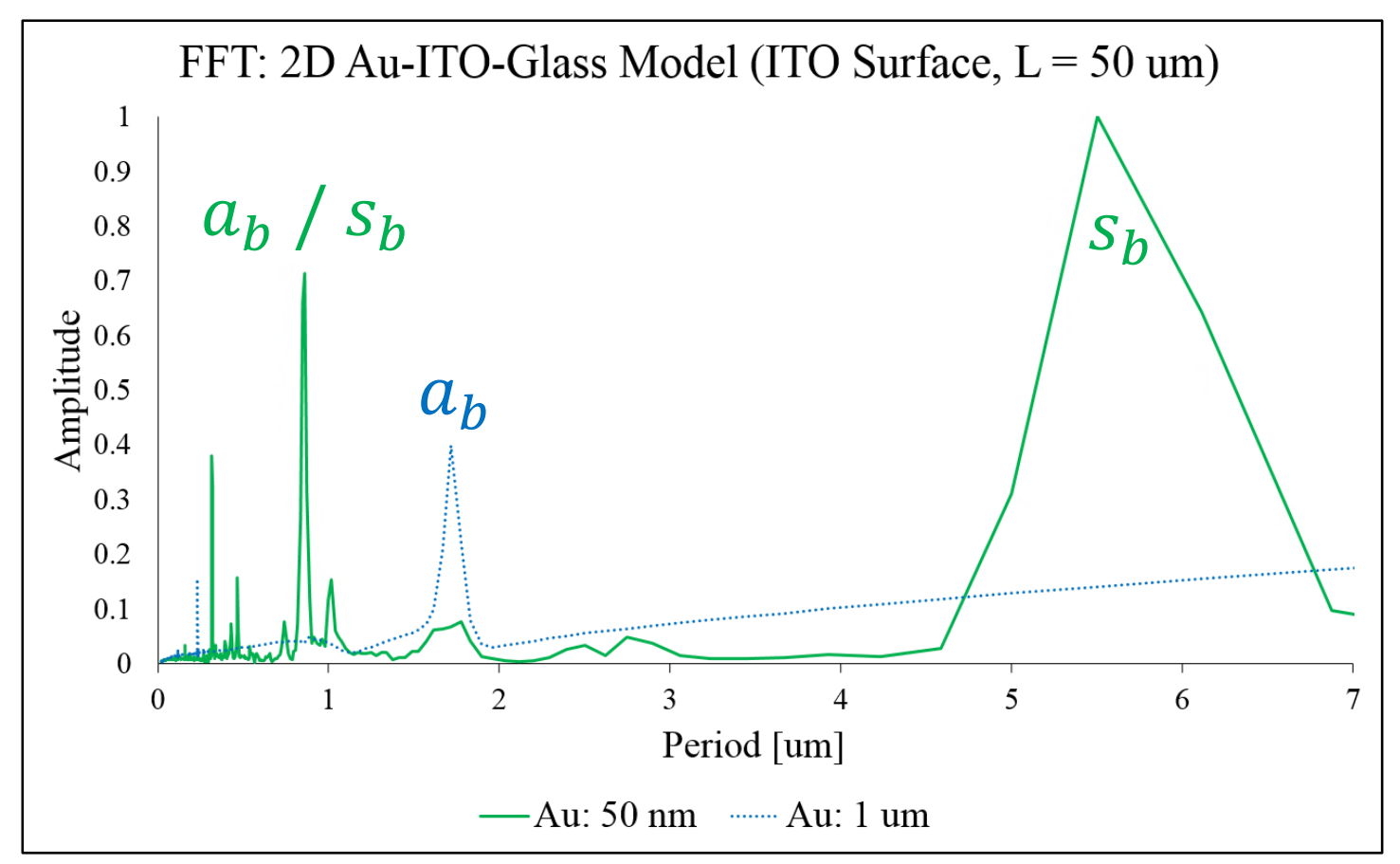

Figure 22: FFT analysis of the electric field data from Figure 21.a taken at the ITO surface. A single periodicity is visible for the $1 \mu \mathrm{m}$ thick gold layer (blue, dotted) at $\sim 1.7 \mu \mathrm{m}$ and two for the $50 \mathrm{~nm}$ thick gold (green, solid) at $\sim 5.5 \mu \mathrm{m}$ and $860 \mathrm{~nm}$.

A summary of the interference spacing periodicities seen in FFT and their attributed constituent modes are provided below in Table 4 . 


\begin{tabular}{|l|l|l|l|c|}
\hline \multicolumn{1}{|c|}{ Surface } & \multicolumn{1}{|c|}{ Interfering Modes } & \multicolumn{1}{c|}{ Theory } & \multicolumn{1}{c|}{ Experiment } & $\begin{array}{c}\text { \% } \\
\text { Diff }\end{array}$ \\
\hline Gold & $\mathrm{s}_{\mathrm{b}} \mathrm{w} /$ oblique, $800 \mathrm{~nm}$ light & $5.18[\mu \mathrm{m}]$ & $5.14 \pm 0.46[\mu \mathrm{m}]$ & 0.81 \\
\hline & Oblique light $\mathrm{w} / \mathrm{sb}$ reflection & $424[\mathrm{~nm}]$ & $424 \pm 3.6[\mathrm{~nm}]$ & 0.05 \\
\hline ITO (thick) & $\mathrm{a}_{\mathrm{b}} \mathrm{w} / \mathrm{ITO}$ light mode & $1.86[\mu \mathrm{m}]$ & $1.72 \pm 0.06[\mu \mathrm{m}]$ & 7.5 \\
\hline ITO (thin) & $\mathrm{s}_{\mathrm{b}}$ w/ oblique, $800 \mathrm{~nm}$ light & $5.18[\mu \mathrm{m}]$ & $5.56 \pm 0.63[\mu \mathrm{m}]$ & 7.3 \\
\hline & $\mathrm{a}_{\mathrm{b}}$ with $\mathrm{s}_{\mathrm{b}}$ & $901[\mathrm{~nm}]$ & $862 \pm 15[\mathrm{~nm}]$ & 4.4 \\
\hline
\end{tabular}

Table 4: Theoretical and experimental values for interference periodicities at the gold and ITO surfaces for thick $(1 \mu \mathrm{m})$ and thin $(50 \mathrm{~nm})$ gold layers. 


\subsubsection{Bounded Surfaces}

Looking at long gold regions is useful for characterizing the unbounded SPP modes for thin and thick layers, but that is not directly relatable to our gold platelets which comprise a bounded surface area. Recall, the triangles of interest primarily range in length from $\sim 3-5 \mu \mathrm{m}$ and therefore the strong surface interference between SPPs excited at the gold surface and obliquely incident light with $\sim 5.2 \mu \mathrm{m}$ periodicity would not be visible for these relatively small sized structures. To evaluate these smaller dimension structures, let us plot the electric field intensity vs. distance for an array of gold lengths such that $\mathrm{L}=\{2.1 \mu \mathrm{m}: 8.0 \mu \mathrm{m}\}$ with $0.1 \mu \mathrm{m}$ steps. Figure 23 (below) shows the electric field intensity at the gold surface for thin (50 nm: green) and thick

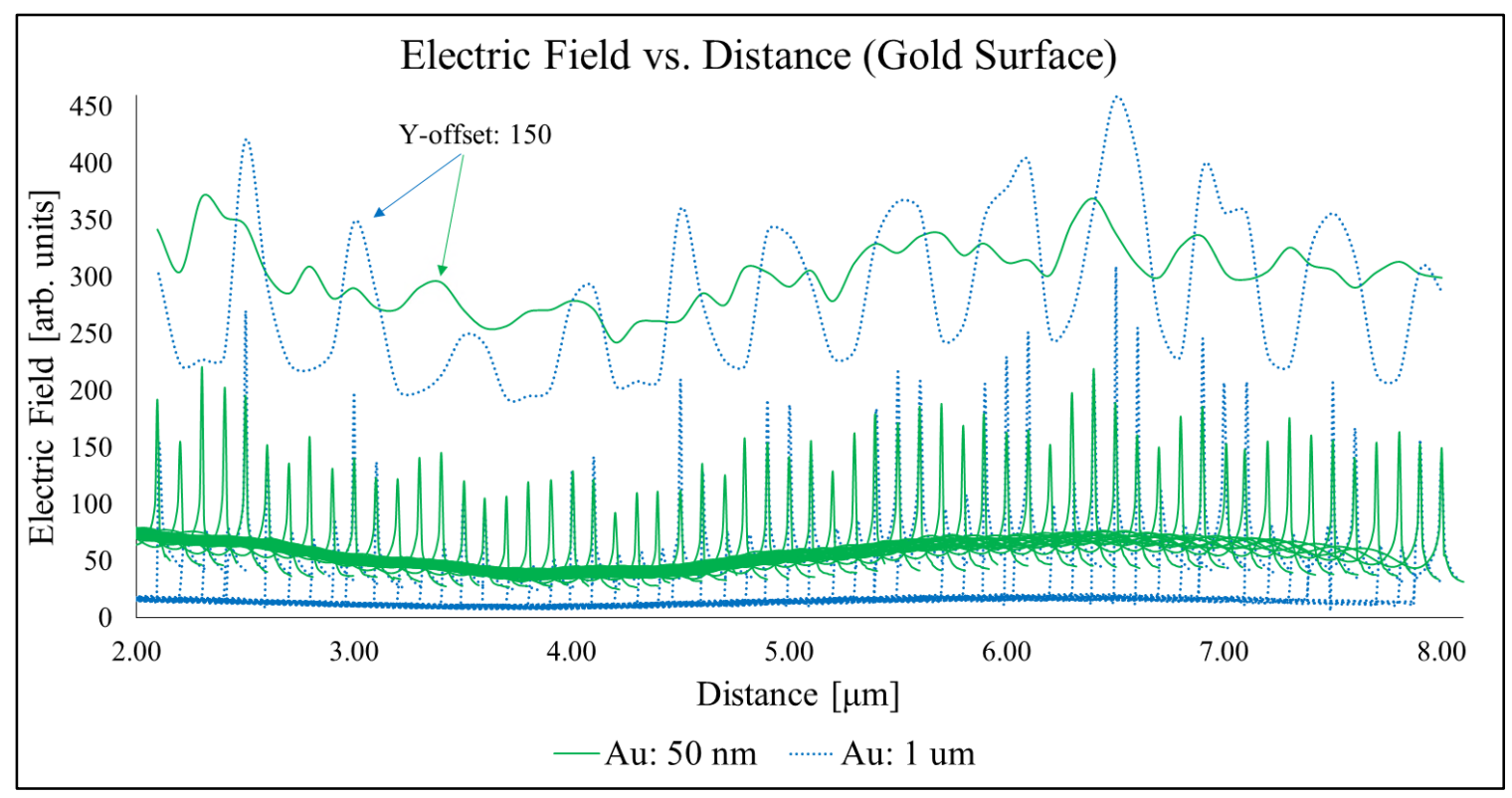

Figure 23: Graph of the time-averaged electric field at the gold surface vs. distance from the front edge of the gold layer for thin $(50 \mathrm{~nm}$; green, solid) and thick ( $1 \mu \mathrm{m}$; blue, dotted) gold platelets of varying length $(\mathrm{L}=2.1 \mu \mathrm{m}-8.0 \mu \mathrm{m})$ exposed to $\lambda=800 \mathrm{~nm}$ light with oblique incidence $\left(\theta=60^{\circ}\right)$ and TM polarization. The electric field intensity at the rear tip for each length is graphed with a vertical translation of $y_{\text {shift }}=150$ for both $50 \mathrm{~nm}$ (green, solid) and $1 \mu \mathrm{m}$ (blue, dotted) thick gold layers. 
( $1 \mu \mathrm{m}$ : black) gold regions. In addition, the maximum electric field intensity for each length (i.e. at the rear tip) is graphed with a translation of $\mathrm{y}_{\text {shift }}=150$ for both layers.

From the data plotted in Figure 23 (above), there are a few trends to note:

(1) the electric field intensity across the gold surface is consistently and significantly higher for the thin $50 \mathrm{~nm}$ gold sample; (2) the rear tip emission has a simpler and larger amplitude of periodicity for the $1 \mu \mathrm{m}$ sample; and (3) we do not see a consistent or even decaying periodicity in the maximum electric field intensity at the tip as the gold layer's length is increased. Let us address these items one at a time:

1. Most of the field strength of the symmetric mode $\left(\mathrm{sb}_{\mathrm{b}}\right)$ is in the vacuum region above the gold surface. As the conducting gold layer becomes thinner, the field strength of the anti-symmetric $\left(a_{b}\right)$ mode becomes stronger at the gold layer. Thus, even though the contribution to the electric field intensity at the gold surface from the $\mathrm{s}_{\mathrm{b}}$ SPP mode remains constant (the wavelength for the $\mathrm{sb}_{\mathrm{b}}$ mode does not change between $\mathrm{T}=50 \mathrm{~nm}: 1 \mu \mathrm{m})$, the increasing $\mathrm{ab}_{\mathrm{b}}$ mode will lead to an overall increase in the electric field intensity at the surface of the gold.

2. For a thick $1 \mu \mathrm{m}$ gold layer, the localized tip emission will predominately be a function of the symmetric $\left(\mathrm{s}_{\mathrm{b}}\right)$ SPP mode and thus we would expect some periodicity. For $\lambda=800 \mathrm{~nm}$ light with gold platelets ranging in length from $\mathrm{L}=\{2: 8 \mu \mathrm{m}\}$ (Figure 23, above), the periodicity of the local maxima in tip emission occurs every $\sim 500 \mathrm{~nm}$. A relatively large variation in the amplitude of the electric field intensity is also reasonable as the emission maxima moves on and off the tip region with a changing gold layer length. For a thin $50 \mathrm{~nm}$ gold layer, the localized tip emission is a 
function of both the $\mathrm{sb}_{\mathrm{b}}$ and anti-symmetric $\left(\mathrm{a}_{\mathrm{b}}\right)$ SPP modes and thus the electric field intensity as a function of platelet length will be more complex. In Figure 23 (above), the periodicity of the local maxima in tip emission is seen to be shorter for a thin gold layer, with a much lower amplitude of variation of the electric field intensity. This may be a product of the two contributing SPP modes having significantly different wavelengths (784 nm vs. $418 \mathrm{~nm}$ ), resulting in a more uniform electric field intensity at the tip region for the entire plotted length range.

3. If this were an unbounded, perfectly conducting gold layer we would expect an undamped periodic electric field intensity across the region. If this gold layer were unbounded, but not perfectly conducting, then the periodicity should remain consistent across the surface, but the amplitude of the electric field intensity should steadily decrease as the SPPs decay due to energy losses to the surrounding materials. This was exactly the case for the electric field of the long gold layers plotted in Figure 23.a (above). In these cases, the unbound (or approximately unbound) gold surface supports a traveling SPP mode which eventually decays. In a bounded, short gold region it is reasonable to expect some reflection off the rear edge from the traveling SPP mode excited at the front edge of the region. If this were the case, we would expect to see the travelling SPP mode interfere with the reflection wave, thus creating a standing wave in the rear region of the gold surface. In Figure 23 (above), we see the electric field intensity at the tip region steadily decrease with increasing gold layer length (due to losses), then increase and decrease again before the rear edge (due to a standing interference wave). As the periodicity of the standing 
interference wave will be a function of the wavelengths of the traveling and reflection modes, this should be independent of the length of the gold region. The percentage of the overall gold surface covered by this standing wave would be a function of the length of the gold region, with the standing wave having an increasing impact on the overall electric field intensity across the gold surface with decreasing gold layer length. 


\subsection{3-Dimensional Simulation Results}

Most of the work in 3-dimensions concerns triangular platelets, although various combinations of rods were used to decompose the overall response into separate contributing effects. Simulations were performed for oblique $\left(\theta=60^{\circ}\right)$ and normal incidence $\left(\theta=0^{\circ}\right)$, the whole polarization spectrum $\left(\varphi=\left\{0: 360^{\circ}\right\}\right)$, and a range of incident light wavelengths $(\lambda=\{625 \mathrm{~nm}: 1 \mu \mathrm{m}\})$. The results will be divided into:

1. Central surface effects

2. External edge effects

3. Tip sharpness

4. Polarization

5. Wavelength

Let us begin with the overall effect seen in the triangular gold platelets. As in the PEEM micrographs, strong localized photoemission can be seen around the tips of the gold platelet for both oblique and normal incidence (Figure 24.a, b; below). The time-averaged electric field squared is plotted as a normalized function of distance across the gold surface (Figure 24.c, below), which highlights the strong electric field at the tip region. For both oblique and normal incidence, the rear tip has the strongest emission, but this effect is even more pronounced for normal incidence. This overall effect has multiple contributing factors, which are detailed in the following sections. 


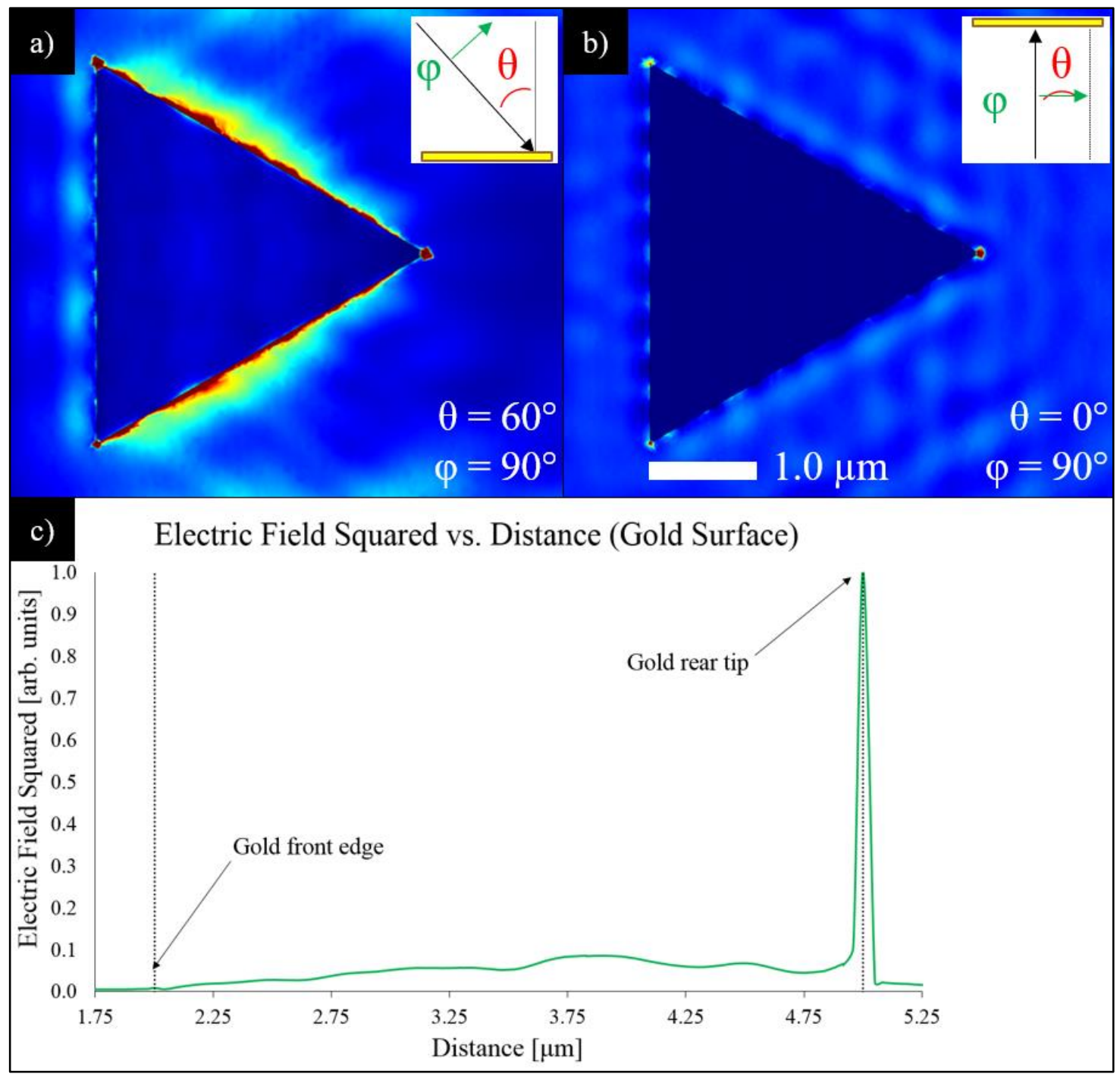

Figure 24: Simulation results of the time-averaged electric field squared at the gold surface for a $3 \mu \mathrm{m}$ long gold platelet exposed to $\lambda=800 \mathrm{~nm}$ light with $\varphi=90^{\circ}$ polarization for: (a) oblique incidence $\left(\theta=60^{\circ}\right)$ and (b) normal incidence $\left(\theta=0^{\circ}\right)$. (c) Graph of the electric field squared vs. distance from the boundary of the model for oblique incidence. Contrast ranges of the micrographs are: $\{$ blue : red $\}=(a)\left\{8 \times 10^{-5}: 149\right\}$ and (b) $\left\{\sim 5 \times 10^{-4}: 20\right\}$. 


\subsubsection{Central surface effects}

To begin, we can show that the standing central mode described above in Section 5.2 for the $2 \mathrm{D}$ sample is also present in a $3 \mathrm{D}$ triangular platelet. As before, due to the small size of the platelet the SPPs excited at the front edge of the gold will travel across the surface of the gold layer and superimpose with a reflection from the end of the bounded gold surface to create a standing mode in the triangle. This standing mode will contribute to the sharp electric field intensity seen in the rear tip region of the triangular platelet, but will be shown to only be one of multiple contributing factors. We also show that the central surface modes are primarily restricted to the surface of the gold region.

First, for a $50 \mathrm{~nm}$ thick gold region, we show that moving into the gold region will lead to a significant decrease in the electric field, down to the order of the surrounding ITO region (Figure 25, below). This incredibly sharp drop off is partially an artifact of the minimum reasonable meshing size, due to the system's memory. It is known that a field at the surface should decrease evanescently into the gold region, as can be seen near the rear tip region [15].

Next, we can show that this central surface mode does not fully account for the overall effect seen in the triangular platelet by approximating the triangle (symmetric in the y-dimension perpendicular to the direction of propagation) as a simplified collection of individual rods (Figure 26, below). We show that to match the electric field profile of the triangular platelet, we must include four individual rods, which approximately represent the three external edges (front, left, and right) and a central region. The rods have the same sharp square edges and thickness as the gold platelet $(50 \mathrm{~nm})$. In addition, 


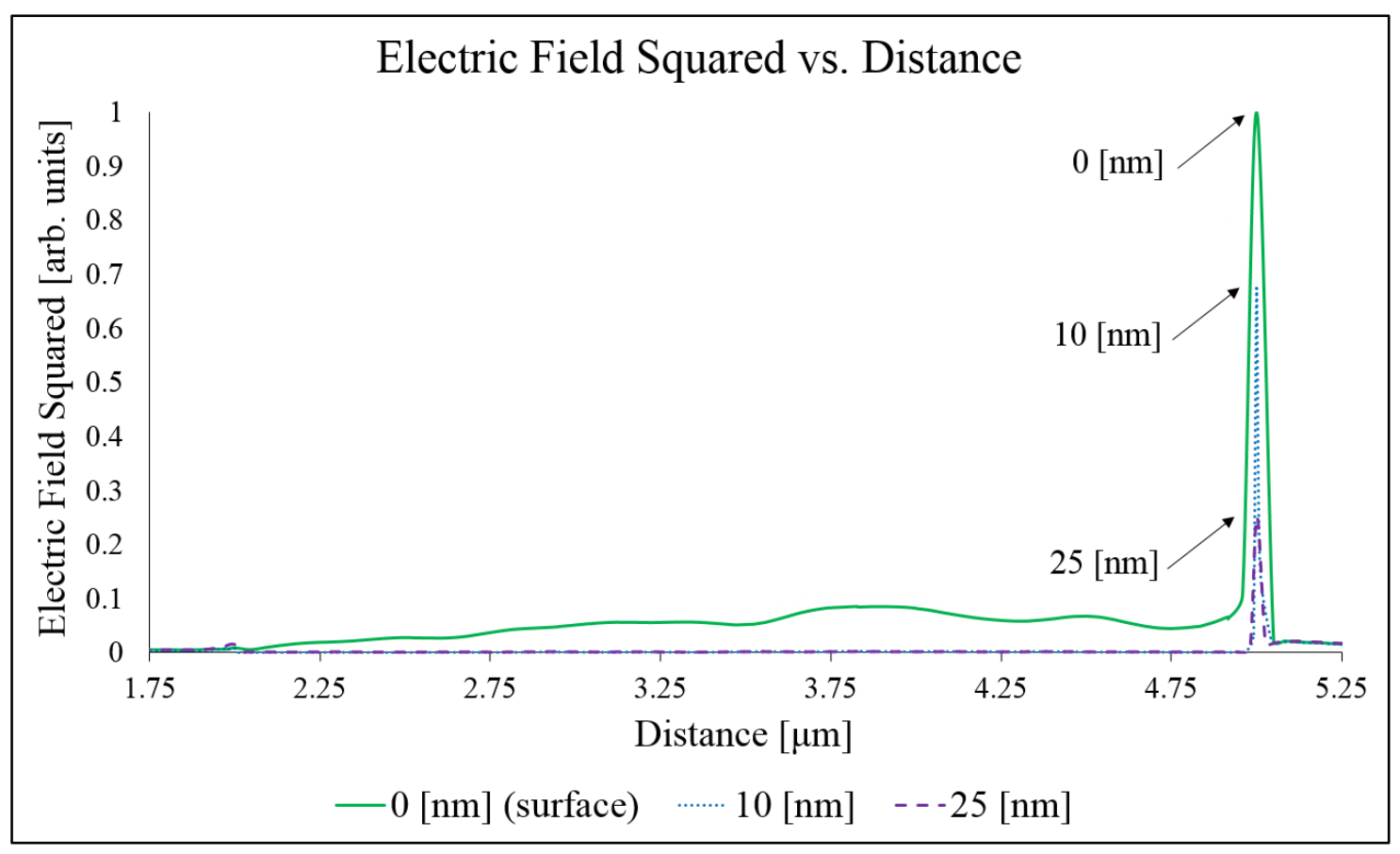

Figure 25: Graph of the time-averaged electric field squared vs. distance from the boundary of the model for a $3 \mu \mathrm{m}$ long gold platelet exposed to $\lambda=800 \mathrm{~nm}$ light with oblique incidence $\left(\theta=60^{\circ}\right)$ and TM polarization for a series of vertical distances into the gold region (i.e. $0[\mathrm{~nm}]=$ gold surface, $25[\mathrm{~nm}]=$ midpoint for $50[\mathrm{~nm}]$ thick layer).

all arrangements have identical front edges and rear tip regions with internal angles of $60^{\circ}$. As seen above, the "Triple + Back" four rod structure will match the simulated electric field response from the triangular platelet. Thus, the sharp electric field intensity at the tip region is not solely the result of a central, standing mode on the gold surface (which was detailed above in Section 5.2.3). 


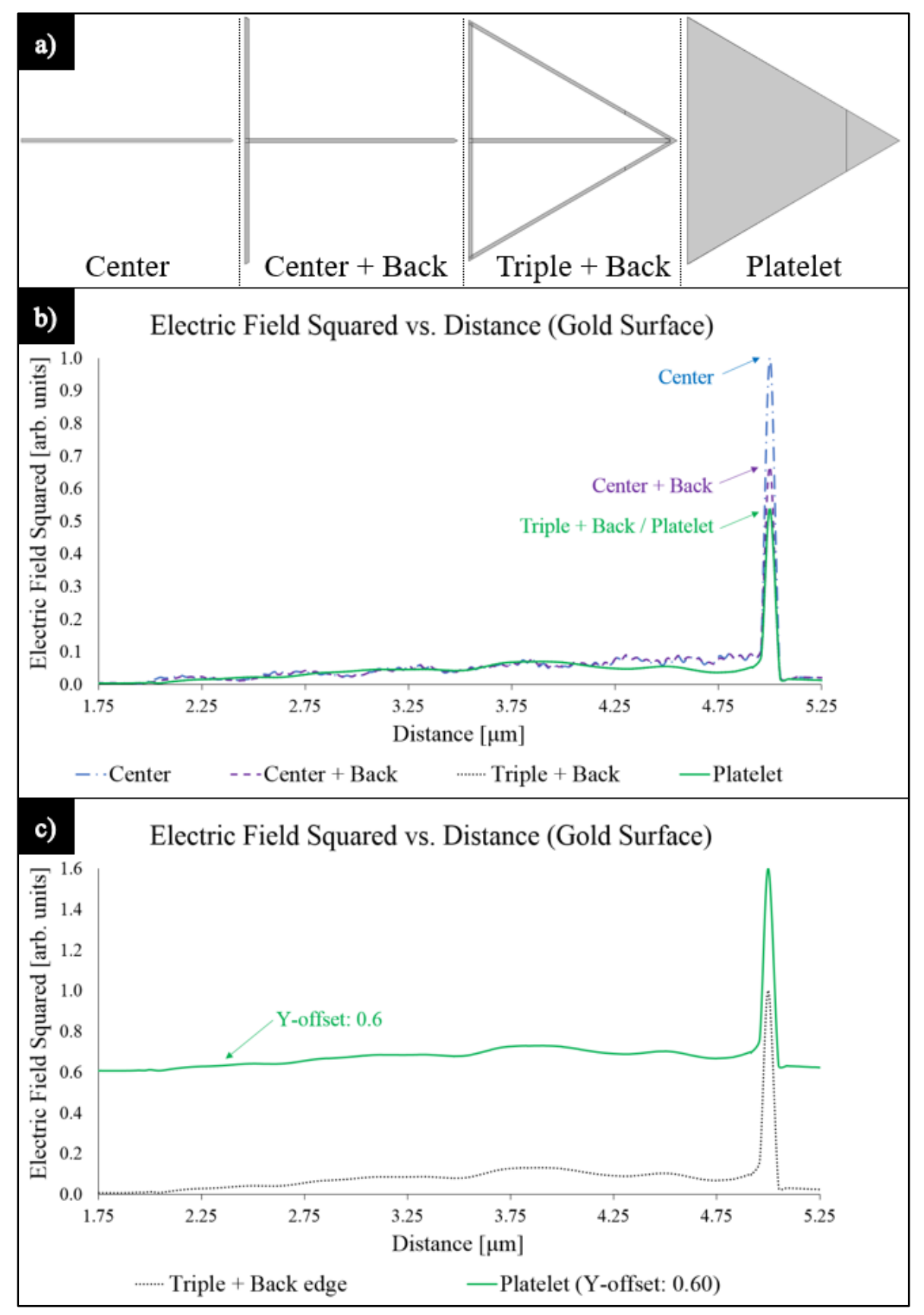

Figure 26: (a) Illustrations of the three combinations of rods and a triangular platelet; (b) a graph of the simulated time-averaged electric field squared at the gold surface vs. distance from the boundary of the model for $\lambda=800 \mathrm{~nm}$ light with oblique incidence $\left(\theta=60^{\circ}\right)$ and TM polarization for the four structures; and (c) the same data graphed for only the triangular platelet (Y-offset: 0.6; green, solid) and the "Triple + back" rod arrangement (black, dotted), showing identical electric field responses. The gold platelet and rod structures are $3 \mu \mathrm{m}$ in length. 


\subsubsection{Edge effects}

In addition to a central standing surface mode, we can show that there are edge modes along the exterior of the gold platelet that will contribute to the overall photoemission response (Figure 27, below). These modes are also measured at the top surface of the gold layer and are symmetric between the left and right edges. To highlight these edge modes, we will again approximate the triangular platelet as an arrangement of rods, beginning with a single angled rod and working up to a three-sided hollow triangle (i.e. three connected rods). As seen below, the "Dual + Back" three rod structure will match the simulated electric field response along the exterior edges from the triangular platelet. As with the standing central surface mode, it is reasonable to expect that a standing mode would also exist along the exterior edges for relatively small platelets on the order of microns. A similarly detailed analysis of the electric field intensity along the exterior edges for a range of edge lengths could be performed to support this hypothesis. Note: The jitters seen in the graphs of Figure 27.b, c (below)—for the regions of $\mathrm{D}=\{\sim 2.25: 3.25 \mu \mathrm{m}$ and $\sim 4.5: 5.0 \mu \mathrm{m}\}$ —are artifacts introduced in the simulation results from the meshing size of the model. 


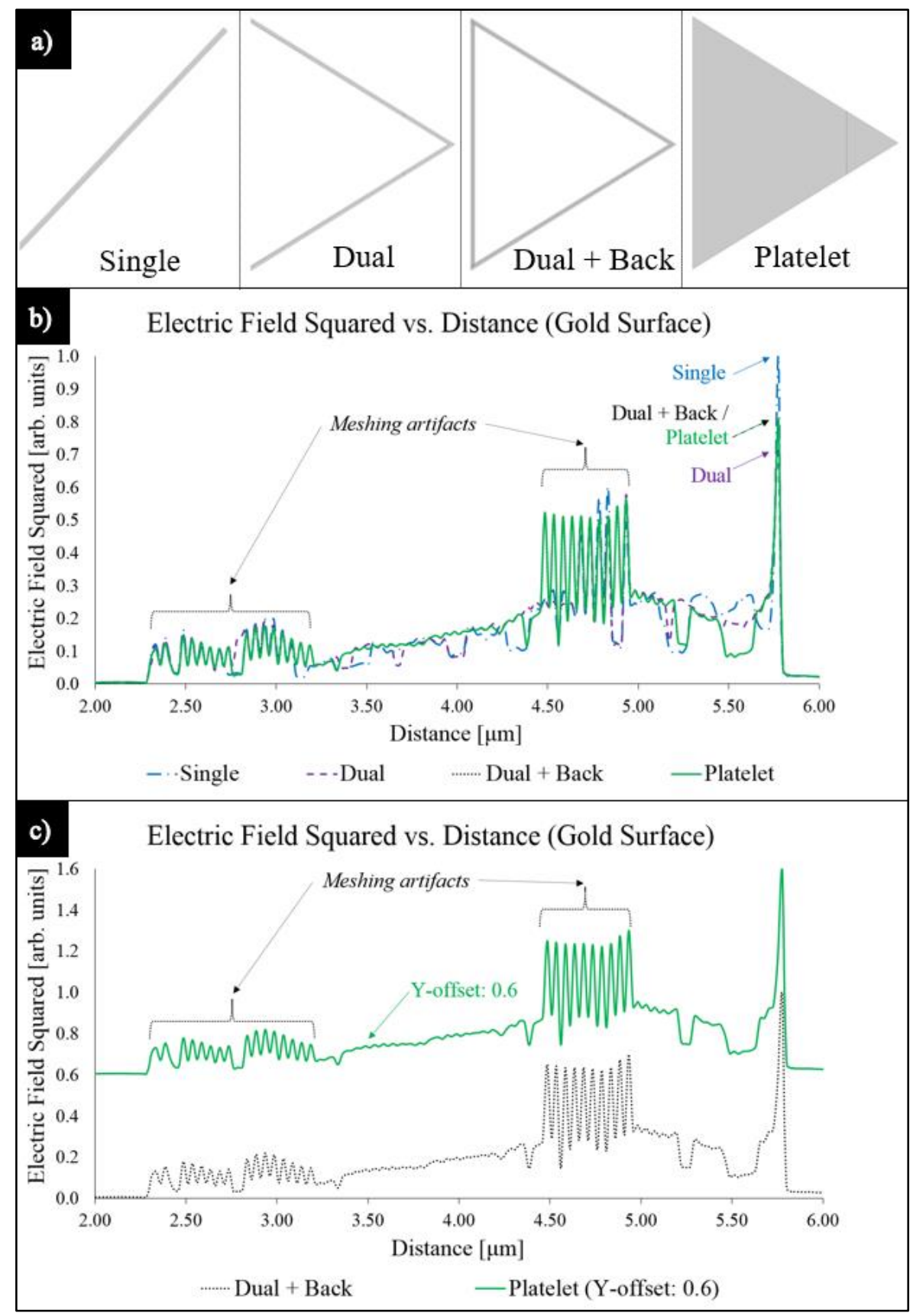

Figure 27: (a) Illustrations of the three combinations of rods and a triangular platelet; (b) a graph of the simulated time-averaged electric field squared at the gold surface vs. distance from the boundary of the model for $\lambda=800 \mathrm{~nm}$ light with oblique incidence $\left(\theta=60^{\circ}\right)$ and TM polarization for the four structures; and (c) the same data graphed for only the triangular platelet (Y-offset: 0.6 ; green, solid) and the "Dual + back" rod arrangement (black, dotted), showing identical electric field responses. The gold platelet and rod structures are $3 \mu \mathrm{m}$ in length. 


\subsubsection{Tip sharpness}

In addition to modes along the center and exterior edges of the triangular platelets, the sharpness of the tip regions will also affect the photoemission. Recall, for a conductor the surface should have a near constant potential, with the local electric field perpendicular to the surface. If a region of a conductor has curvature, the local electric field is expected to be stronger than that of a surrounding flat region. A sharp edge (e.g. tip of a triangle), would represent an area of very high curvature. Thus, we would expect the local electric field to increase the higher the curvature.

Simulation results of the time-averaged electric field squared are presented for $\lambda=800 \mathrm{~nm}$, obliquely incident $\left(\theta=60^{\circ}\right), \mathrm{TM}$ polarized light on a single angled rod structure for varying tip angles. We show that varying the sharpness of the (rear) tip significantly affects the magnitude of the localized electric field, by a factor of $4.4: 1$ $\left(19.5: 1\right.$ for $\left.|\overrightarrow{\mathrm{E}}|^{2}\right)$ for an internal angle range of $30^{\circ}-90^{\circ}$. A magnified view of the tip

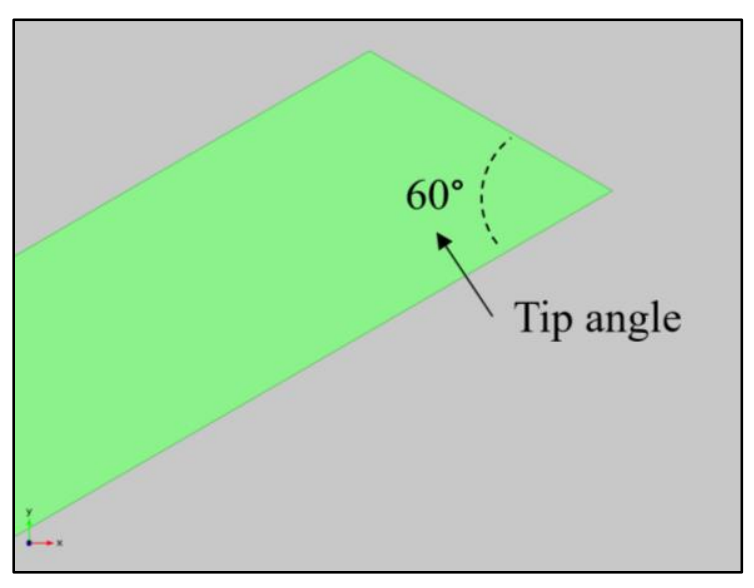

Figure 28: Illustration of a gold rod (highlighted in green) with the tip angle labeled. 
region, highlighting the relationship between the sharpness (i.e. smaller internal angle) and the magnitude of the electric field (squared) is shown in Figure 29 (below). For the triangular platelets, which are approximately equilateral in geometry, each tip will have an internal angle of $\sim 60^{\circ}$. Due to this relatively sharp edge, each tip region will display photoemission, although the effect is significantly less than that of the propagating central or edge modes on the surface of the gold region.

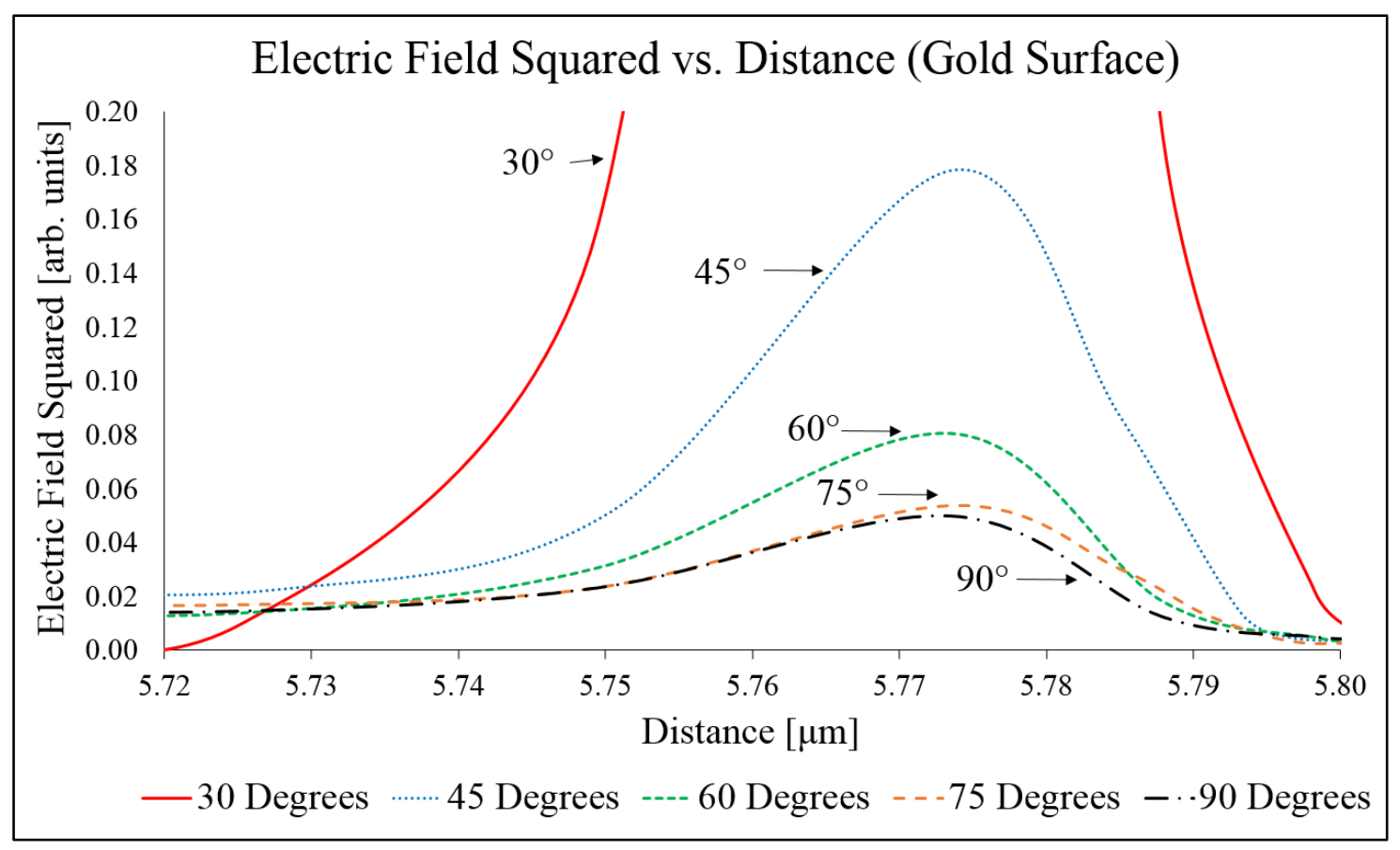

Figure 29: Graph of the simulated time-averaged electric field squared at the gold surface vs. distance from the boundary of the model for $\lambda=800 \mathrm{~nm}$ light with: oblique incidence $\left(\theta=60^{\circ}\right)$ and TM polarization for single angled rods with varying internal tip angle between $\left\{30^{\circ}: 90^{\circ}\right\}$. Magnified view of the tip region. Note: the data is normalized such that the peak electric field intensity at the tip for the $30^{\circ}$ internal angle is 1 . The electric field outside of the region displayed is approximately zero for all internal angles. 


\subsubsection{Polarization}

Simulation results of the time-averaged electric field squared are presented for obliquely incident $\left(\theta=60^{\circ}\right)$ light on a single triangular gold platelet in the two polarization extremes: $\mathrm{TM}$ mode $\left(\varphi=90^{\circ}\right.$ or $\left.270^{\circ}\right)$ and TE mode $\left(\varphi=0^{\circ}\right.$ or $\left.180^{\circ}\right)$

(Figure 30.a, b; below). Simulation results for normal incidence $\left(\theta=0^{\circ}\right)$ in its two polarization extremes: $\varphi=90^{\circ}$ and $\varphi=0^{\circ}$ are also presented (Figure 30.c, d; below).

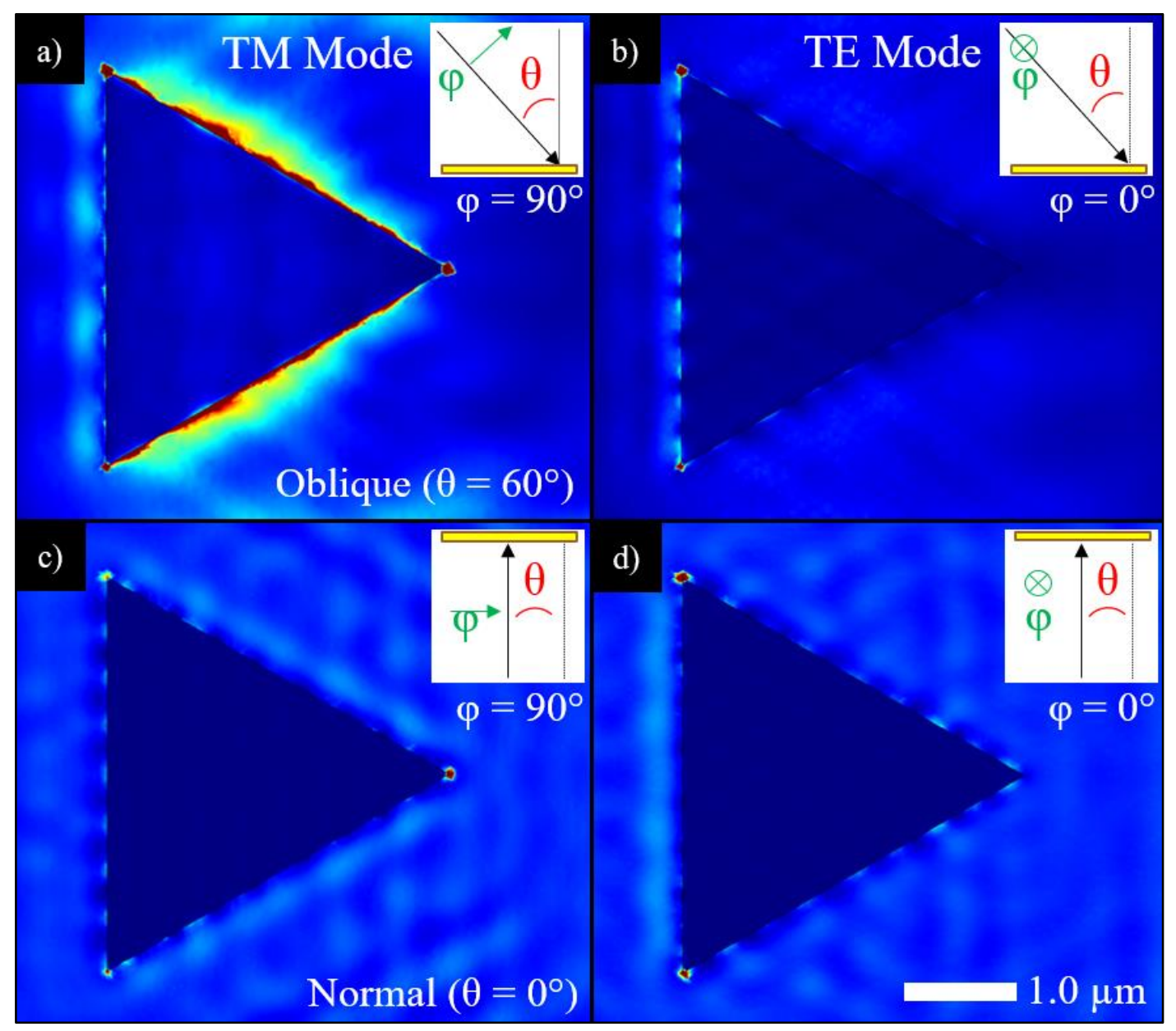

Figure 30: Simulation results of the time-averaged electric field squared at the gold surface for $3 \mu \mathrm{m}$ long gold platelets exposed to $\lambda=800 \mathrm{~nm}$ light with: oblique incidence $\left(\theta=60^{\circ}\right)$ (a) TM and (b) TE and normal incidence $\left(\theta=0^{\circ}\right)$ (c) $\varphi=90^{\circ}$ and (d) $\varphi=0^{\circ}$ polarizations. Contrast ranges of the micrographs are: $\{$ blue $:$ red $\}=$ (a) $\left\{8 \times 10^{-5}: 149\right\}$, (b) $\left\{8 \times 10^{-5}: 33\right\}$, and (c, d) $\left\{\sim 5 \times 10^{-4}: 20\right\}$. 
If we plot the electric field data vs. the distance across the gold surface, we can perform quantitative comparisons between the polarizations and incident angles

(Figure 31, below). As can be seen from the graph, there is near zero photoemission from the oblique TE and normal $\varphi=0^{\circ}$ polarizations for the rear tip region.

Lastly, we can plot the electric field magnitude at the rear tip region for $\lambda=800 \mathrm{~nm}$ light with oblique incidence $\left(\theta=60^{\circ}\right) \mathrm{vs}$. the polarization angle for the whole spectrum of $\left(\varphi=\left\{0: 360^{\circ}\right\}\right)$ (Figure 32, below). As seen in the graph, the polarization dependence for oblique incidence can be closely approximate by an absolute sine function of the polarization angle $\varphi$.

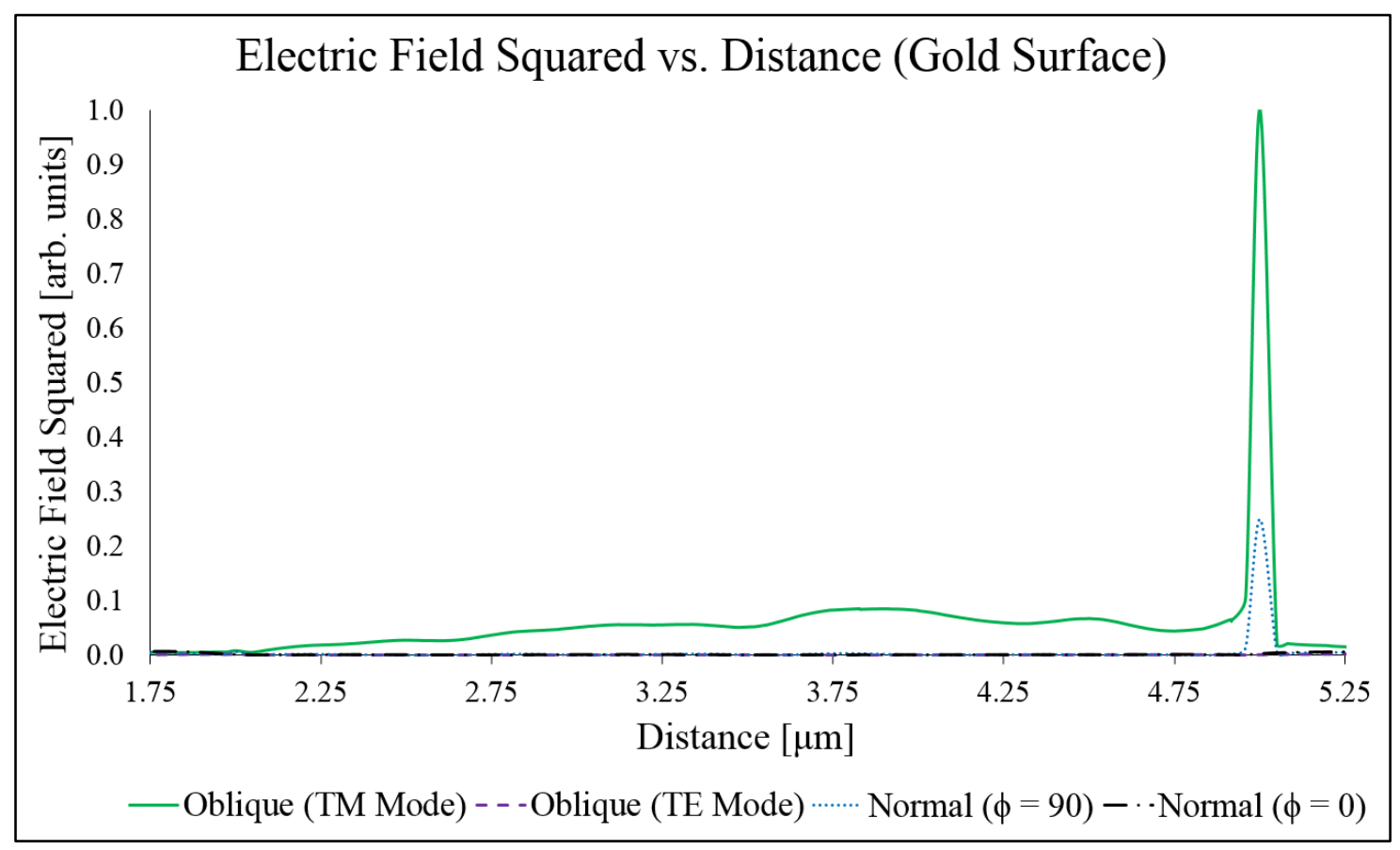

Figure 31: Graph of the simulated time-averaged electric field squared at the gold surface vs. distance from the boundary of the model for a $3 \mu \mathrm{m}$ long gold platelet exposed to $\lambda=800 \mathrm{~nm}$ light with: oblique incidence $\left(\theta=60^{\circ}\right)$ for TM $\left(\varphi=90^{\circ}\right.$ or $270^{\circ}$; green, solid $)$ and TE $\left(\varphi=0^{\circ}\right.$ or $180^{\circ}$; purple, dashed $)$ polarizations and normal incidence for $\varphi=90^{\circ}$ (blue, dotted) and $\varphi=0^{\circ}$ (black, mixed) polarizations. Note: Oblique TE and normal $\varphi=0^{\circ}$ show no photoemission. 


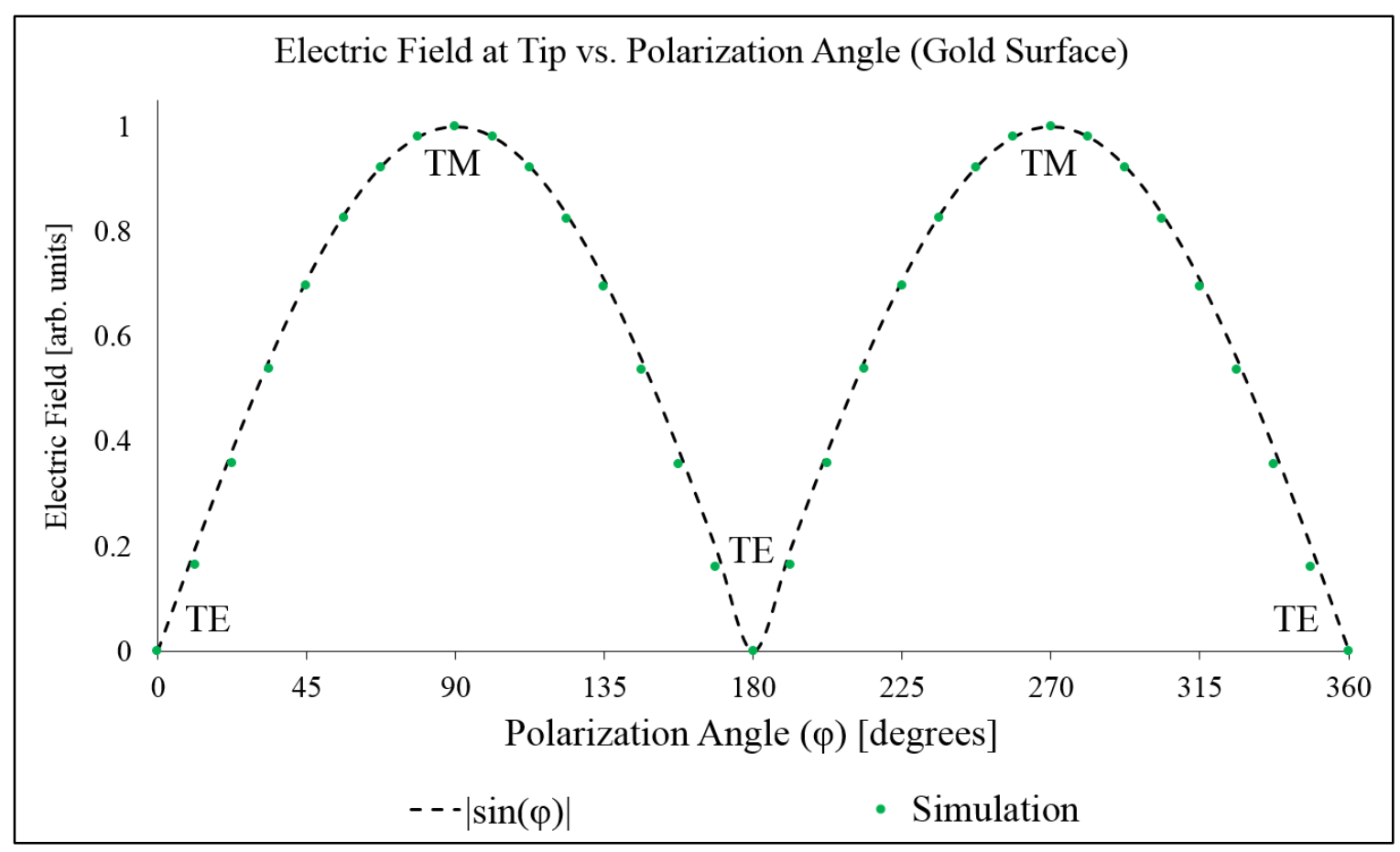

Figure 32: Graph of the simulated time-averaged electric field squared at the gold surface of the rear tip vs. polarization angle for obliquely incident light $\left(\theta=60^{\circ}\right)$ for $\varphi=\left\{0: 360^{\circ}\right\}$. 


\subsubsection{Wavelength}

Simulation results of the time-averaged electric field squared are presented for obliquely incident $\left(\theta=60^{\circ}\right)$ light on a single triangular gold platelet for a range of laser wavelengths $(\lambda=\{625 \mathrm{~nm}: 1 \mu \mathrm{m}\})$ centered around the illumination wavelength of $\lambda=800 \mathrm{~nm}$ from the experimental PEEM results. Figure 33 (below) shows the simulated field responses for the two wavelength extremes: $\lambda=625 \mathrm{~nm}$ and $1 \mu \mathrm{m}$. If we plot the electric field data at the rear tip vs. the wavelength of the incident light, we can quantitatively compare the photoemission across the wide spectrum of photon energies (Figure 34, below). In the graph, the fundamental wavelength range of the PEEM's pulsed Ti:Sapphire laser is highlighted for reference.

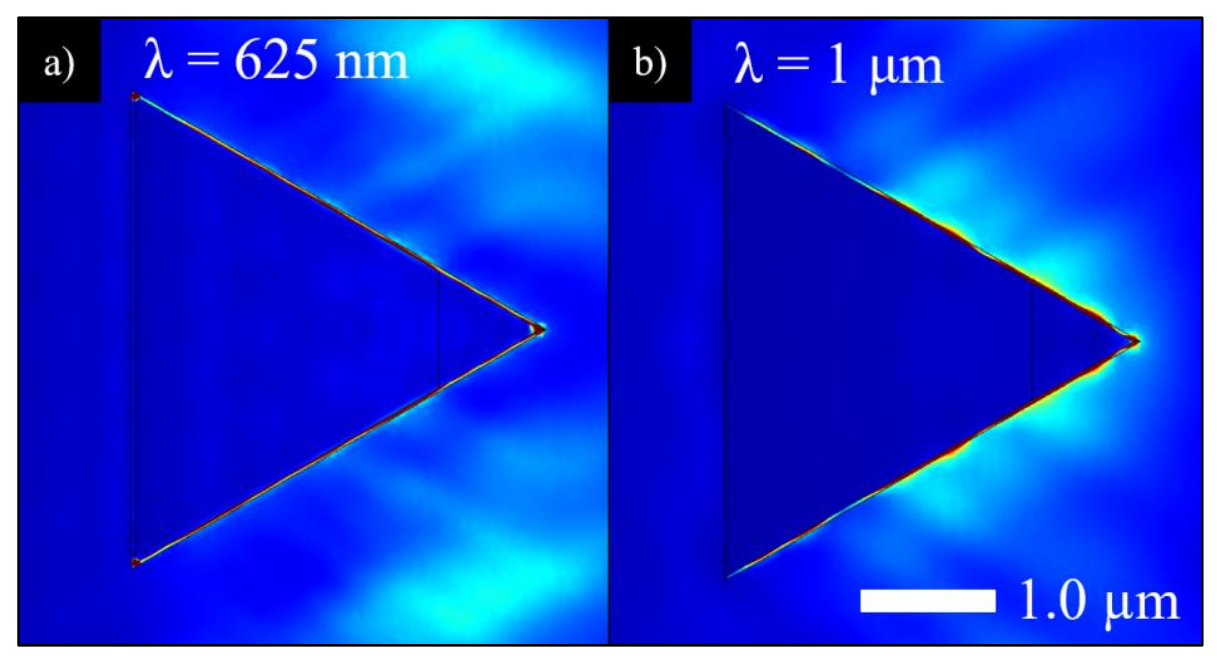

Figure 33: Simulation results of the time-averaged electric field squared at the gold surface for $3 \mu \mathrm{m}$ long gold platelets exposed to $\lambda=800 \mathrm{~nm}$ light with oblique incidence $\left(\theta=60^{\circ}\right) \mathrm{TM}$ polarization for (a) $\lambda=625 \mathrm{~nm}$ and (b) $\lambda=1 \mu \mathrm{m}$. Contrast ranges of the micrographs are: $\{$ blue $:$ red $\}=\left(\right.$ a) $\left\{9.8 \times 10^{-4}: 708\right\}$ and (b) $\left\{2 \times 10^{-3}: 264\right\}$. 


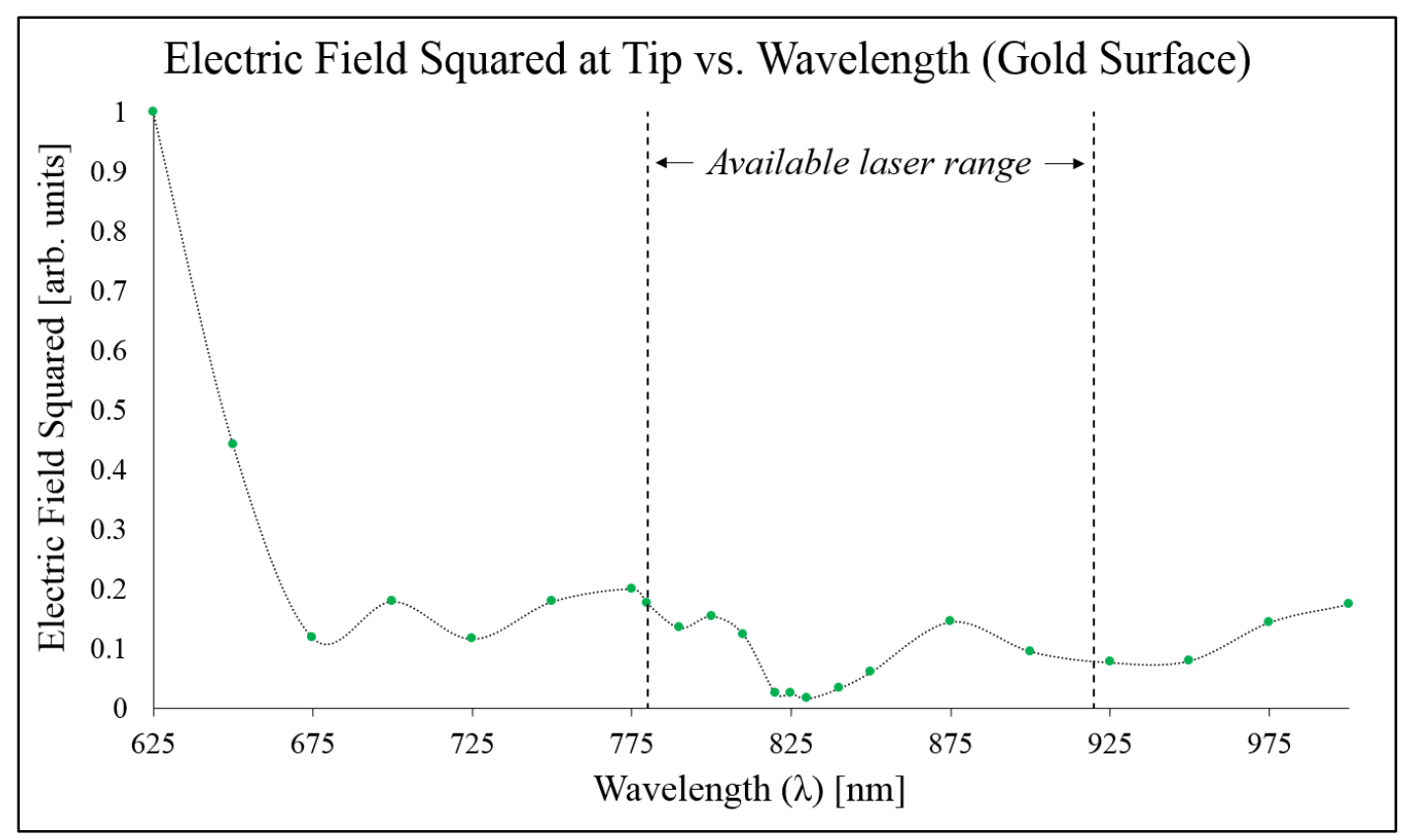

Figure 34: Graph of the simulated time-averaged electric field squared at the gold surface of the rear tip vs. wavelength for a $3 \mu \mathrm{m}$ long gold platelet exposed to obliquely incident light $\left(\theta=60^{\circ}\right)$ for $\lambda=\{625 \mathrm{~nm}: 1 \mu \mathrm{m}\}$. Note: The fundamental wavelength for the PEEM's pulsed laser is marked. 


\subsection{Experiment vs. Simulation}

The simulation results have approximately reproduced the localized photoemission seen in PEEM micrographs, for both the strong rear tip and weaker edge responses (Figure 30.a, b; above). The diameter of the localized tip emission is significantly smaller than both the overall dimensions of the gold platelet $(\sim 3 \mu \mathrm{m})$ and the wavelength of the incident light $(800 \mathrm{~nm})$ and in this result is bounded to within $\sim 250 \mathrm{~nm}$. As seen in Figure 30.a (above), the simulated tip emission is confined even further to within $150 \mathrm{~nm}$. As with the experimental results, for oblique incidence localized photoemission is seen in the triangular platelets for TM mode polarization, but not for TE mode. Quantitative comparisons between the two extremes can be made in a plot of the time-averaged electric field squared across the gold surface vs. distance from the model boundary (Figure 31, above). Graphically, for TM polarization we see the strong field at the tip region, as well as a much lower field across the gold surface.

There are a few notable differences between the simulated triangular platelet and that imaged in the PEEM. In Figure 35 (below), a direct comparison highlights the following differences: (1) the size of the platelets are slightly different, (2) the offset angle of the triangle in the PEEM was not built into the COMSOL model, (3) the sharper edges in the tip regions of the model produce stronger responses along the front edge of the platelet, and (4) the exact thicknesses of the experimental gold platelets are unknown. The thickness of the platelets are $\sim 50 \mathrm{~nm}$ and presumed to be $50 \mathrm{~nm}$ for the simulations [16]. It is not shown here, but the thickness of the gold platelet will have an impact on the 
magnitude of the localized photoemission. All in all, the effects seen in PEEM are still reproduced in the simulation results, even with these noted differences.

For normal incidence, the simulation results are also well aligned. The detailed and periodic edge emission seen in PEEM is reproduced, as well as the polarization dependence. The simulation results again show strong localized photoemission at the tip regions, which is not seen in PEEM for normal incidence. This is attributed to the sharp, rectangular edges used in the model compared to the curved edges of the gold samples imaged in PEEM (as discussed above in Section 5.3.3). In addition, the normal incidence micrographs were of a different gold platelet sample than the oblique micrographs.

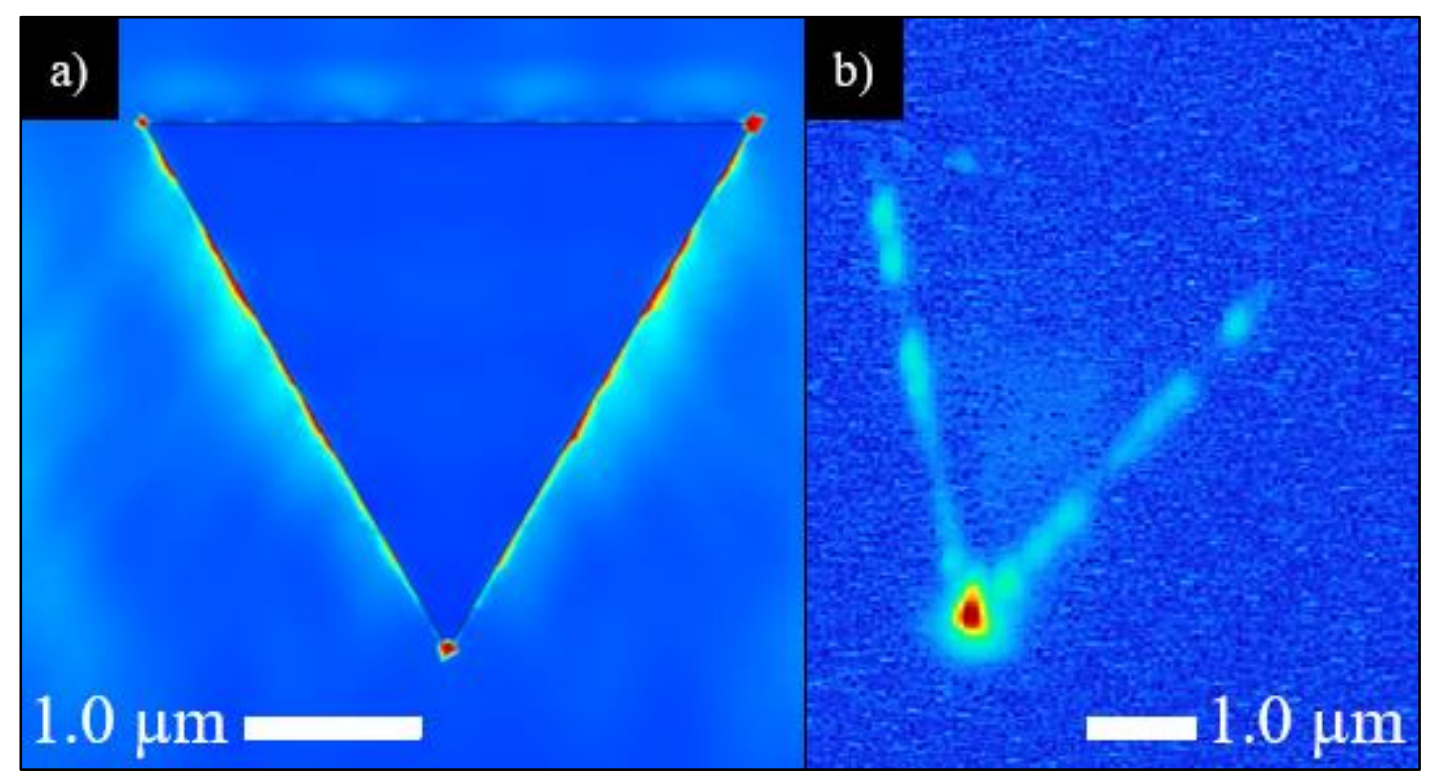

Figure 35: (a) Simulation results of the time-averaged electric field squared at the triangular gold surface for $\lambda=800 \mathrm{~nm}$ light with oblique incidence $\left(\theta=60^{\circ}\right)$ and $\mathrm{TM}$ polarization; (b) PEEM micrograph (false color) with the same conditions. Contrast ranges of the micrographs are: $\{$ blue : red $\}=$ (a) $\left\{8.0 \times 10^{-5}: 149\right\}$ and (b) $\{1256: 40875\}$. Note: (b) is presented as the $4^{\text {th }}$ root of the photoemission yield. Original PEEM micrograph for (b) provided by Dr. Robert C. Word. 


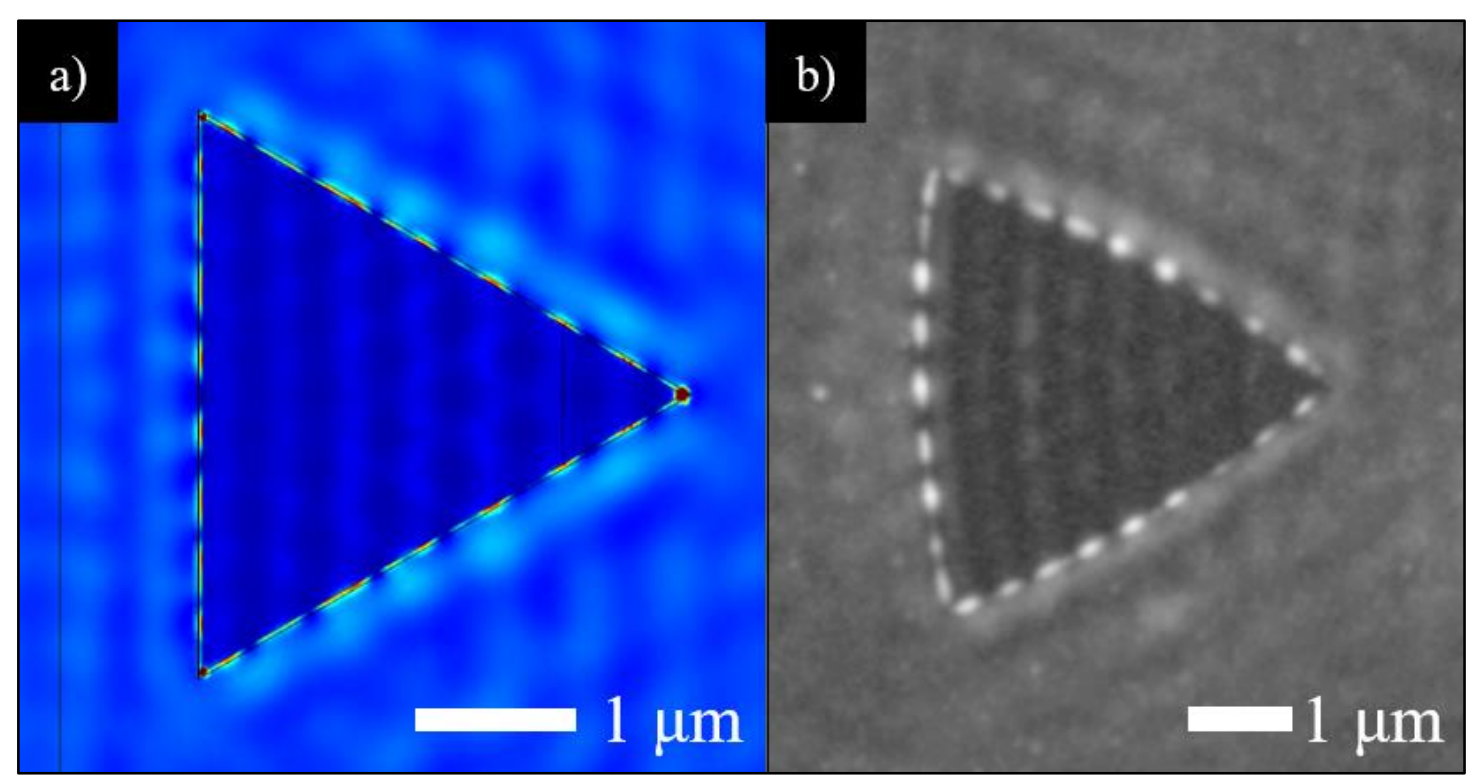

Figure 36: Simulation results of the time-averaged electric field squared for a triangular gold layer ( $35 \mathrm{~nm}$ below the gold surface, $15 \mathrm{~nm}$ above the ITO surface) for $\lambda=800 \mathrm{~nm}$ light with normal incidence $\left(\theta=0^{\circ}\right)$, TM polarization (a) and PEEM micrograph with the same conditions (b). Contrast range of the colored image (a) is:

$\{$ blue : red $\}=\left\{6.8 \times 10^{-3}: 1572\right\}$. Note: The micrograph (b) is presented as the $3^{\text {rd }}$ root of the photoemission yield.

- Image (b) adapted with permission from Photonic and plasmonic surface field distributions characterized with normal- and oblique-incidence multi-photon PEEM, by R.C. Word and R. Könenkamp, Ultramicroscopy, vol. 183, pp. 1139-1351, 2017 [26].

Figure 36 (above) shows a direct comparison of the normal incidence simulation results and an associated PEEM micrograph. Note that Figure 36.a shows the time-averaged electric field squared $35 \mathrm{~nm}$ below the gold surface ( $15 \mathrm{~nm}$ above the ITO surface), to more closely match the edge emission seen in PEEM (Figure 36.b). This implies PEEM imaging is primarily due to the anti-symmetric SPP mode $\left(a_{b}\right)$, which agrees with previously published findings [19]. As we can see in Figure 37 (below), this is due both to the $a_{b}$ mode having large confinement near the gold surface and its high intensity relative to the symmetric mode $\left(\mathrm{s}_{\mathrm{b}}\right)$. In addition, the length of the triangles studied here is much shorter than the expected interference periodicity. For longer 
triangles, it may be possible to see the sb SPP mode in PEEM, but its intensity would still be significantly lower than that of the $a_{b}$ mode.

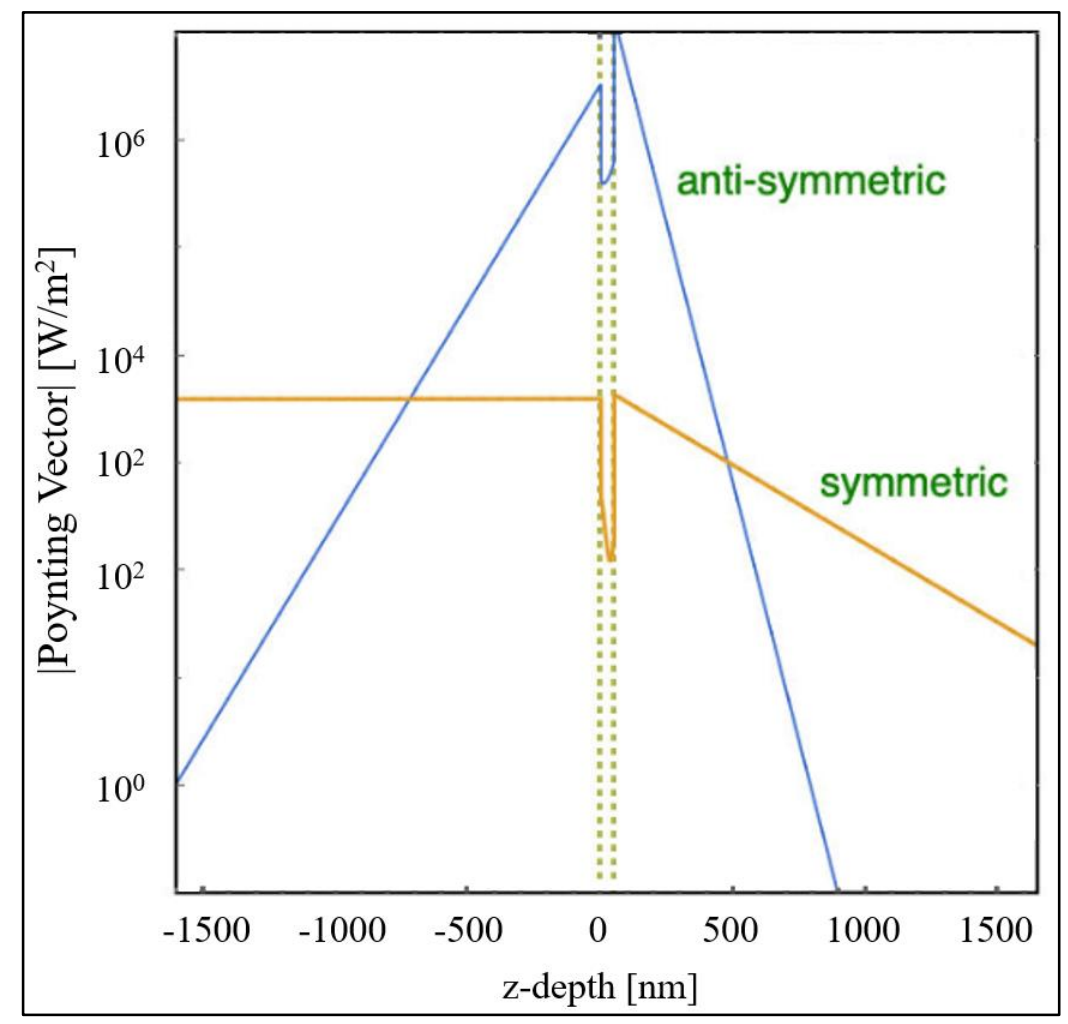

Figure 37: Plot showing the magnitude of the Poynting vector vs. vertical depth $(\mathrm{z})$ for the symmetric and anti-symmetric SPP modes for $\lambda=800 \mathrm{~nm}$ light obliquely incident $\left(\theta=60^{\circ}\right)$ for a vacuum-gold-ITO sample with $50 \mathrm{~nm}$ thick gold. Plot courtesy of Dr. Robert C. Word. 


\subsection{Dependencies of Localized Photoemission}

\subsubsection{Polarization}

In the experiments presented here, an electric field across a conducting metal surface results from incident laser light diffracting at the front edge of a triangular gold platelet, leading to the excitation of surface plasmon polariton (SPP) modes in the conducting layer. A basic qualitative explanation is to consider the gold surface as an infinite, conducting plane. In this case, an electric field parallel to the gold surface cannot be sustained and therefore will not contribute to electron excitation in the material. For oblique-incidence, only pure-TE modes have electric fields entirely parallel to the surface, thus excitation in the gold would be expected for all other polarizations. This model will eventually fail for small (i.e. finite) areas and structures. This breakdown is clearly seen at normal incidence $\left(\theta=0^{\circ}\right)$, where the electric field for all polarizations is in-plane to the sample, yet localized plasmon resonances are weakly excited at the edges. In this case, strong localized fields can be produced at two of the corners of the triangle by adjusting the polarization angle (Figure 30.c, d; above). In general, for an equilateral triangular platelet, the two tips which are closest in line with the direction of the polarization will demonstrate strong emission, while the third tip will have somewhat weaker emission. In the simulation results the situation is very similar: localized fields are at lower intensities for those offset from the polarization direction; which is primarily due to the sharp tip edges where weak localized surface plasmon resonances can be induced. While this selectivity is noteworthy, the plasmonic coupling efficiency for an 
in-plane electric field is significantly weaker than for an oblique field with some perpendicular component. Comparing the maximum field strength (squared) at the rear tip for the extreme incidences (oblique : normal), we note a factor of $4: 1$ difference in the photoemission yield assuming a 4-photon process.

For obliquely-incident $\left(\theta=60^{\circ}\right)$ TE polarized light the electric field across the gold surface is near zero, with the strongest fields concentrated at the front two tips. Photoemission here is again a function of tip sharpness, where weak localized surface resonances can be induced. For the rear tip, we note from Figure 31 (above) that the electric field squared for TM polarization is $\sim 600 \mathrm{X}$ larger than for TE.

With COMSOL models established, we can investigate the overall polarization dependence of the photoemission from the triangular platelets. Adjusting the polarization angle over the whole range of $\varphi=\left\{0: 360^{\circ}\right\}$-in increments of $11.25^{\circ}$-we see that the magnitude of the electric field at the tip region closely follows an (absolute) $|\sin (\varphi)|$ function (Figure 32, above). This is not surprising as the electric field of the incident light perpendicular to the gold surface is proportional to $\sin (\varphi)$, but this polarization dependence clearly suggests that the surface acts as a receiver for the tip emission.

It has been previously shown that by changing the polarization of the incident light, localized photoemission in a gold structure can be effectively switched on or off [32]. In the work presented here we have shown this effect in experiment and simulation, characterized the polarization dependence of the location of strong tip emission, and fit the relationship between the polarization angle $\varphi$ and the magnitude of the localized tip emission to a $|\sin (\varphi)|$ function. 


\subsubsection{Wavelength}

We showed above that both surface and edge modes on the conducting metal layer significantly contribute to the localized photoemission seen in the triangular gold platelets. The number of excited surface plasmon polaritons (SPPs), the energy of the SPPs, and their propagation characteristics are all dependent on the wavelength of the incident laser light. Within the evaluated range of wavelengths, where $\lambda=\{625 \mathrm{~nm}: 1 \mu \mathrm{m}\}$, varying the wavelength will significantly affect the magnitude of the electric field at the rear tip by a factor of $7.9 \mathrm{X}$ between maximum and minimum values $\left(62.5 \mathrm{X}\right.$ for $\left.|\overrightarrow{\mathrm{E}}|^{2}\right)$. The maximum field response occurs for $625 \mathrm{~nm}$, which was the shortest wavelength that could be simulated (due to memory limitations) with the triangular platelet model matching the PEEM results (i.e. $3 \mu \mathrm{m}$ flake length). The minimum field strength occurs $\sim 830 \mathrm{~nm}$, which although it still shows photoemission at the rear tip, the magnitude is effectively zero $(1.6 \%)$ when compared to the maximum field strength at $625 \mathrm{~nm}$. Like the polarization dependence, varying the wavelength can effectively switch the emission on or off, even with the wavelength remaining inside the SPP excitation range for the vacuum-gold-ITO configuration.

Specifically, the simulation results show that within the available $\sim 10 \%$ laser range of the PEEM, varying the wavelength could provide a factor of 3.3 increase in the electric field at the tip region, corresponding to a factor of $\sim 11$ increase in the electric field squared, a factor of $~ 121$ increase for 2-photon emission. By modulating the wavelength from our observed minimum at $830 \mathrm{~nm}$ to the maximum at $625 \mathrm{~nm}$, the photoemission rate for a 2-photon process is predicted to increase by a factor of almost 
4K. Thus, wavelength change at readily available optical frequencies is highly effective and feasible for photoemission in these antennas.

Unlike the polarization dependence, modulating the wavelength within this relatively small range of values will not greatly affect the spatial distribution of the electric field along the exterior edges of triangular platelets of the order of microns in size. As noted above, the triangles characterized in this work are so small that the localized tip emission is predominately a function of standing waves through the center and (presumably standing) waves along the exterior edges of the platelet. The standing wave patterns essentially form a resonance pattern across the triangular platelet and Figure 34 (above) shows a semi-periodic relationship between the magnitude of the electric field (squared) at the rear tip and the wavelength of the incident light. These patterns would inherently be a function of the wavelength of the incident light, the lateral dimensions of the platelet, and the thickness of the platelet. Due to these dependencies, it is reasonable that the details of this resonance pattern would be quite complicated and thus a simple, direct mathematical relationship between the localized photoemission of the tip region and the wavelength of the incident light would not exist; unlike with the polarization dependence. Although we have not derived a direct relationship between the two quantities, it is expected that the maxima in the resonance pattern would be separated by a distance on the order of $\lambda / L$, where $L$ is the length of the gold platelet.

As the characteristics of the modes are dependent on the wavelength of the light and the length of the platelet, for a fixed polarization there should be a unique wavelength which would produce a maximum tip emission for each differently sized platelet. 


\section{Conclusion}

Non-linear PEEM imaging of gold micro-platelets is a powerful tool for characterizing the plasmonic responses with sub-wavelength resolution. Flexibility in the angle of incidence and the wavelength and polarization of the incident laser light make PEEM a useful system for a wide variety of applications. Combined with finite element analysis, detailed quantitative analysis can be predictively performed over a large range of parameter variations in a relatively short amount of time, before attempting any experiments in the laboratory.

The triangular gold platelets characterized in this work are uniformly illuminated from the relatively large laser spot and thus could be used individually or as a collection of electron-emitters or optical antenna within a larger device. Characterization of surface plasmon polariton excitation, SPP modes of propagation, and methods for controlling them are critical for the overall development and improvement of plasmonic devices. Optical antenna are currently under investigation for many possible applications, including: photodetection, photon / electron emission, photovoltaics, information transfer and / or processing, nonlinear signal conversion, probes for nano-imaging, and for biological sensing and detection [43]. In addition, plasmonic-based devices may become critical elements of optical circuits, thus there is great interest from government, academia, and private industry to further this field of study and its application.

In the work presented here, we have demonstrated strong, localized photoemission within a region far smaller than the wavelength of the incident light ( $250 \mathrm{~nm}$ vs. $800 \mathrm{~nm}$ for oblique incidence). This effect has been reproduced in 
simulation and modeled for normal $\left(\theta=0^{\circ}\right)$ and oblique $\left(\theta=60^{\circ}\right)$ incident light, the whole spectrum of polarization of the incident light $\left(\varphi=\left\{0: 360^{\circ}\right\}\right)$, and for a range of different wavelengths $(\lambda=\{625 \mathrm{~nm}: 1 \mu \mathrm{m}\})$. We show that the magnitude of the emission closely follows an absolute $\sin (\varphi)$ dependence on the polarization angle $(\varphi)$ of the incident light, with the ability to switch the localized photoemission at a tip region on or off. Maximum emission occurs for purely-TM polarized light and minimum emission for TE light.

In addition, we show that the localized photoemission in the tip region is the result of SPP modes along the surface and edges of the gold platelet, combined with the sharp, finite geometry of the triangular tips. The central surface mode for these small platelet geometries is a standing wave, the result of interference between the traveling SPPs from the front edge of the gold layer and subsequent reflection waves from the rear of the platelet. The overall tip emission is less intense than the sum of the individual contributions and thus the resultant photoemission involves a somewhat complicated (net constructive) superposition of the different processes.

For continuation of this work, additional effort would be needed to mathematically describe the relationship between the maximum electric field in the tip region and the wavelength of the incident light. In addition, the full possible range of wavelengths which could demonstrate localized photoemission in triangular gold platelets of a realistic size has not been determined. The mathematical models for simulation of the hybridized SPP modes could be advanced to better account for the asymmetric dielectric surroundings, specifically their thicknesses and absorption. Lastly, 
improvements to the 3D COMSOL model could make the approximations more closely resemble experiment, but limitations in the available system memory and computer processing power must be taken into account. Considering the strong approximation of a simplified, single-layer metal-dielectric interface with separate top and bottom SPP modes - in comparison to the more complex, multi-layer dielectric-metal-dielectric interfaces with hybridized symmetric $\left(\mathrm{s}_{\mathrm{b}}\right)$ and anti-symmetric modes $\left(\mathrm{a}_{\mathrm{b}}\right)$ - the rate of return on additional advancements to this treatment is difficult to access.

In conclusion, our results show that the localized tip emission is maximized if the electric field is along the central axis (i.e. TM polarization) and is strongly dependent on the polarization and wavelength of the incident light. 


\section{References}

[1] "Plasmon," www.wikipedia.org, 2018. [Online]. Available: https://en.wikipedia.org/wiki/Plasmon.

[2] T. W. Ebbesen, H. J. Lezec, H. F. Ghaemi, T. Thio, and P. A. Wolff, "Extraordinary optical transmission through sub-wavelength hole arrays," Nature, vol. 391, no. 6, p. 667, 1998.

[3] M. Müller, V. Kravtsov, A. Paarmann, M. B. Raschke, and R. Ernstorfer, "Nanofocused Plasmon-Driven Sub-10 fs Electron Point Source," ACS Photonics, vol. 3, no. 4, pp. 611-619, 2016.

[4] L. Minai, A. Zeidan, D. Yeheskely-Hayon, S. Yudovich, I. Kviatkovsky, and D. Yelin, "Experimental proof for the role of nonlinear photoionization in plasmonic phototherapy," Nano Lett., vol. 16, no. 7, pp. 4601-4607, 2016.

[5] W. P. Putnam, R. G. Hobbs, P. D. Keathley, K. K. Berggren, and F. X. Kärtner, "Optical-field-controlled photoemission from plasmonic nanoparticles," Nat. Phys., vol. 13, no. 4, pp. 335-339, 2017.

[6] S. B. D. Borah, T. Bora, S. Baruah, and J. Dutta, "Groundwater for Sustainable Development Heavy metal ion sensing in water using surface plasmon resonance of metallic nanostructures," Groundw. Sustain. Dev., vol. 1, no. 1-2, 2016.

[7] A. Yanai and U. Levy, "The role of short and long range surface plasmons for plasmonic focusing applications,” Opt. Express, vol. 17, no. 16, p. 14270, 2009.

[8] S. V. Boriskina, H. Ghasemi, and G. Chen, "Plasmonic materials for energy: From physics to applications," Mater. Today, vol. 16, no. 10, pp. 375-386, 2013.

[9] R. Vogelgesang and A. Dmitriev, "Real-space imaging of nanoplasmonic resonances," Analyst, vol. 135, no. 6, pp. 1175-1181, 2010.

[10] D. Pines and D. Bohm, “A Collective Description of Electron Interactions: Collective vs. Individual Particle Aspects of the Interactions," Phys. Rev., vol. 85, no. 338, 1952.

[11] J. D. Jackson, Classical Electrodynamics: 10.8 Plasma Oscillations, 2nd Editio. New York, NY: John Wiley \& Sons, 1975.

[12] R. H. Ritchie, "Plasma Losses by Fast Electrons in Thin Films," Phys. Rev., vol. 106, no. 5, pp. 874-881, 1957. 
[13] "Surface plasmon," www.wikipedia.org, 2018. [Online]. Available: https://en.wikipedia.org/wiki/Surface_plasmon.

[14] "Surface plasmon polariton," www.wikipedia.org, 2018. [Online]. Available: https://en.wikipedia.org/wiki/Surface_plasmon_polariton.

[15] S. A. Maier, Plasmonics: Fundamentals and Applications. Springer Science + Business Media LLC, 2007.

[16] Z. Guo, Y. Zhang, Y. DuanMu, L. Xu, S. Xie, and N. Gu, "Facile synthesis of micrometer-sized gold nanoplates through an aniline-assisted route in ethylene glycol solution," Colloids Surfaces A Physicochem. Eng. Asp., vol. 278, no. 1-3, pp. 33-38, 2006.

[17] S. Dickreuter, J. Gleixner, A. Kolloch, J. Boneberg, E. Scheer, and P. Leiderer, "Mapping of plasmonic resonances in nanotriangles," Beilstein J. Nanotechnol., vol. 4, no. 1, pp. 588-602, 2013.

[18] B. Frank et al., "Electrochemical Route to Large-Area Mono-Crystalline Gold Platelets for High-Quality Plasmonic Applications," Adv. Photonics, 2014.

[19] R. C. Word, J. P. S. Fitzgerald, and R. Könenkamp, "Direct imaging of optical diffraction in photoemission electron microscopy," Appl. Phys. Lett., vol. 103, no. 2, p. 21118, 2013.

[20] M. Aeschlimann et al., "Optimal open-loop near-field control of plasmonic nanostructures," New J. Phys., vol. 14, 2012.

[21] B. Frank, "Short-Range Surface Plasmonics on Single Crystalline Gold Platelets," University of Stuttgart, 2016.

[22] D. Podbiel et al., "Imaging the Nonlinear Plasmoemission Dynamics of Electrons from Strong Plasmonic Fields," Nano Lett., vol. 17, no. 11, pp. 6569-6574, 2017.

[23] V. Flauraud et al., "In-Plane Plasmonic Antenna Arrays with Surface Nanogaps for Giant Fluorescence Enhancement," Nano Lett., vol. 17, no. 3, 2017.

[24] D.-B. Li et al., "Direct observation of localized surface plasmon field enhancement by Kelvin probe force microscopy," Light Sci. Appl., vol. 6, no. 8, 2017.

[25] Z. Li, C. Xu, W. Liu, M. Li, and X. Chen, "Nonlinear inelastic electron scattering from $\mathrm{Au}$ nanostructures induced by localized surface plasmon resonance." 2018. 
[26] R. C. Word and R. Könenkamp, "Photonic and plasmonic surface field distributions characterized with normal- and oblique-incidence multi-photon PEEM," Ultramicroscopy, vol. 183, pp. 1339-1351, 2017.

[27] P. Kahl et al., "Direct Observation of Surface Plasmon Polariton Propagation and Interference by Time-Resolved Imaging in Normal-Incidence Two Photon Photoemission Microscopy,” Plasmonics, vol. 13, no. 1, pp. 239-246, 2018.

[28] B. Ji et al., "Control and mapping ultrafast plasmons with PEEM," no. November 2016, p. 100280G, 2016.

[29] J. P. S. Fitzgerald, R. C. Word, and R. Könenkamp, "Subwavelength visualization of light in thin film waveguides with photoelectrons," Phys. Rev. B - Condens. Matter Mater. Phys., vol. 89, no. 19, 2014.

[30] T. A. Stenmark, "Photoemission Electron Microscopy for Analysis of Dielectric Structures and the Goos-Hänchen Shift," Portland State University, 2016.

[31] R. Könenkamp, R. C. Word, G. F. Rempfer, T. Dixon, L. Almaraz, and T. Jones, "5.4Nm Spatial Resolution in Biological Photoemission Electron Microscopy," Ultramicroscopy, vol. 110, no. 7, pp. 899-902, 2010.

[32] R. C. Word, J. P. S. Fitzgerald, and R. Könenkamp, "Direct coupling of photonic modes and surface plasmon polaritons observed in 2-photon PEEM," Opt. Express, vol. 21, no. 25, pp. 30507-30520, 2013.

[33] "Finite difference method," www.wikipedia.org, 2018. [Online]. Available: https://en.wikipedia.org/wiki/Finite_difference_method.

[34] J. von Neumann and R. Richtmyer, "A method for the numerical calculation of hydrodynamic shocks," J. Appl. Phys., vol. 21, pp. 232-237, 1950.

[35] "Finite element method," www.wikipedia.org, 2019. [Online]. Available: https://en.wikipedia.org/wiki/Finite_element_method\#Basic_concepts.

[36] W. Frei, "Using Perfectly Matched Layers and Scattering Boundary Conditions for Wave Electromagnetics Problems," COMSOL Blog, 2015. [Online]. Available: https://www.comsol.com/blogs/using-perfectly-matched-layers-and-scatteringboundary-conditions-for-wave-electromagnetics-problems/.

[37] P. B. Johnson and R. W. Christy, "Optical constants of the noble metals," Phys. Rev. B, vol. 6, no. 12, pp. 4370-4379, 1972. 
[38] R. J. Moerland and J. P. Hoogenboom, "Subnanometer-accuracy optical distance ruler based on fluorescence quenching by transpartent conductors," Optica, vol. 3, pp. 112-117, 2016.

[39] I. H. Matlitson, "Interspecimen compariosn of the refractive index of fused silica," J. Opt. Soc. Am., vol. 55, pp. 1205-1208, 1965.

[40] A. Yariv and P. Yeh, Photonics: Optical Electronics in Modern Communications, 6th ed. New York: Oxford University Press, 2007.

[41] H. Raether, Surface Plasmons on Smooth and Rough Surfaces and on Gratings, no. 1. Berlin: Springer-Verlag, 1988.

[42] "Knife-edge effect," www.wikipedia.org, 2017. [Online]. Available: https://en.wikipedia.org/wiki/Knife-edge_effect.

[43] L. Novotny and N. Van Hulst, "Antennas for light," Nat. Photonics, vol. 5, no. 2, pp. 83-90, 2011. 